\title{
STRATEGIES FOR TARGETED IMAGING OF LEUKOCYTES IN THE LUNG
}

A Dissertation Presented to

The Faculty of the School of Engineering and Applied Science

University of Virginia

\author{
In Partial Fulfillment \\ of the requirements for the Degree \\ Doctor of Philosophy in Biomedical Engineering
}

By

Landon W. Locke

May 2012 


\section{Approval Sheet}

The dissertation is submitted in partial fulfillment of the requirements for the degree of

Doctor of Philosophy in Biomedical Engineering

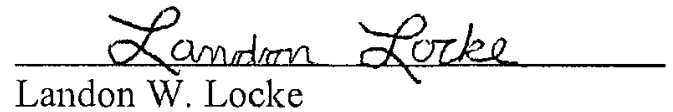

This dissertation has been read and approved by the examining Committee:

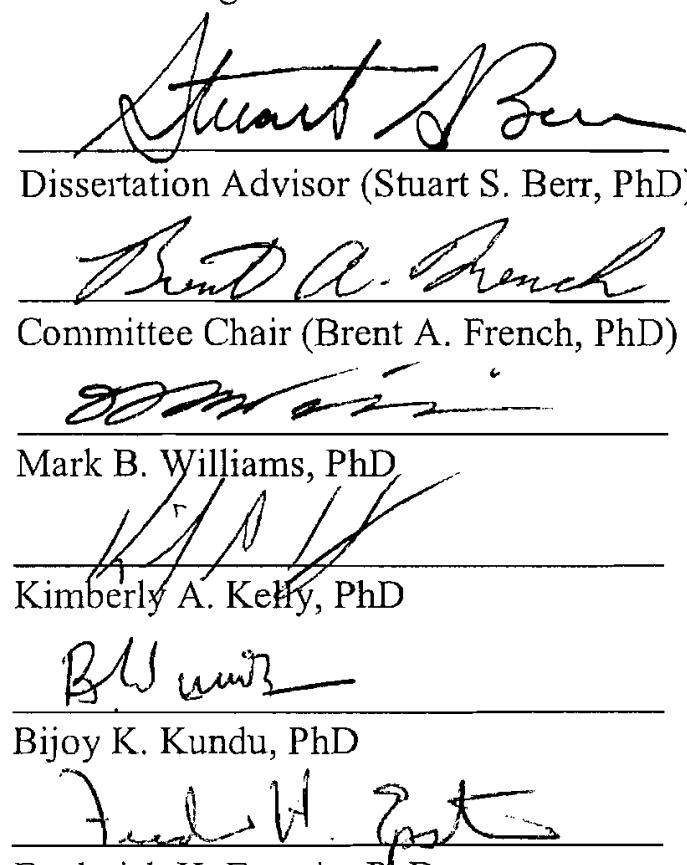

Frederick H. Epstein, PhD

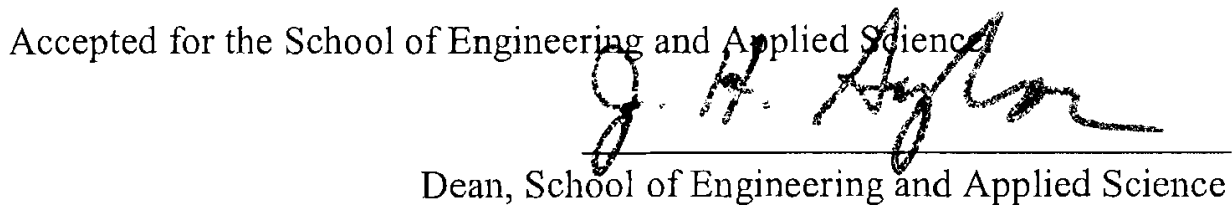

May 2012 


\section{Abstract}

The pulmonary inflammatory response is a tightly regulated multicellular process geared towards protecting the host from invading organisms, yet chronic and exuberant inflammation is a hallmark of many pulmonary diseases including lung cancer, chronic obstructive pulmonary disease (COPD), and asthma. Neutrophils and macrophages are consistently identified as key effector cells in most pulmonary inflammatory diseases. As a result, there has been great effort directed towards understanding the roles of these leukocytes in the context of debilitating lung diseases. In the work presented here, we have focused on the development of molecular imaging based on Positron Emission Tomography (PET) that may facilitate noninvasive evaluation and monitoring of leukocytes in the lung.

I first explored the use of $2^{\prime}$-deoxy-2'-[ $\left[{ }^{18}\right.$ F $]$ fluoro-D-glucose (FDG)-PET with compartmental model analysis to probe lung neutrophil metabolic activity. The difficulty with this approach in small animal applications is the requirement for measuring the blood input function during the PET scan, traditionally obtained by invasive means. Therefore, I have: 1) established a noninvasive method for deriving the blood input function directly from gated, dynamic PET images; and 2) determined the feasibility and accuracy of measuring neutrophil metabolic activity with FDG-PET by threecompartment model analysis with an image-derived input function in a mouse model of acute pulmonary inflammation. Based on estimates of the model parameters, the net FDG uptake $\left(\mathrm{K}_{\mathrm{i}}\right)$ was estimated in lungs following bacteria challenge and was found to be 2.24 times higher compared to controls. Importantly, the mean lung $\mathrm{K}_{\mathrm{i}}$ value computed here was not significantly different from previously reported lung values 
computed from input functions obtained by physical arterial sampling, validating our noninvasive approach.

The second strategy was based on the peptide N-cinnamoyl-F-(D)L-F-(D)L-F (cFLFLF), which was previously reported to be an antagonist to the neutrophil formyl peptide receptor (FPR). We demonstrated that the bioavailability and blood clearance properties of the peptide ligand could be improved by polyethyleneglycol (PEG) conjugation without adversely affecting its binding affinity or neutrophil stimulatory properties. In vivo PET imaging was conducted in mice and the results of these studies demonstrated that this modified peptide has excellent potential for monitoring neutrophilic inflammation in the lungs and may be valuable in evaluating future antinflammatory therapies.

In addition to neutrophils, there is also much incentive to develop noninvasive molecular imaging agents that target macrophages. Macrophages, which play an important role promoting inflammatory disorders of the lung, consistently have been shown to exhibit predominantly a M2-like phenotype which can facilitate the growth and spread of lung tumors. The final chapter of this dissertation documents my attempts to target tumor-associated macrophages (TAMs) using liposomes surface-coated with a mannose ligand. This liposome modification has been shown to improve M2 macrophage recognition and internalization via mannose receptor-mediated endocytosis but has not been studied as a macrophage targeting agent for PET imaging applications. Initial studies conducted by us in vitro confirmed that mannosylated $\left(\mathrm{Man}_{3}\right)$-liposomes were significantly taken up more by M2 (IL4/13-stimulated) bone marrow-derived macrophages following incubation compared to M1 (LPS/IFN- $\gamma$-stimulated) cells. In 
vivo PET imaging studies were conducted in a mouse model of chemically-induced lung cancer and revealed that $\mathrm{Man}_{3}$-liposomes exhibited a 7-fold higher uptake in tumor tissue compared to normal lung tissue $6 \mathrm{~h}$ after intravenous injection. Subsequent ex vivo lung biodistribution and confocal microscopy studies confirmed uptake of $\mathrm{Man}_{3}$-liposomes by TAMs. Finally, co-injection studies revealed that $\mathrm{Man}_{3}$-liposomes exhibited the highest tumor contrast relative to normal lung tissue at the time point studied compared to plain or PEGylated liposomes. 


\section{Acknowledgements}

This dissertation is dedicated to the memory of Dr. Walt Rogers.

I first want to thank my wife Dr. Jenifer Warner-Locke for helping me get through difficult times and always showing incredible patience. You have helped me make this dissertation possible and I love you very much. To my Mom, thank you for your words of encouragement and inspiring confidence in me. To my Dad and my sister, thank you both for your support and love. This dissertation would not have been possible without the support and guidance of my advisor Dr. Stuart Berr. Thank for you believing in me. I will always be grateful for the opportunity to have worked with you.

I want to thank the members of my dissertation committee, Dr. Brent French, Dr. Bijoy Kundu, Dr. Mark Williams, Dr. Frederick Epstein, and Dr. Kimberly Kelly for generously giving their time and expertise to better my work. I am especially grateful to Dr. Mark Williams for generously providing financial support for the manufacturing of liposomes as well to Dr. Sasha Klibanov for preparing liposomes and lending his expertise in deciphering experimental data. I am also indebted to Dr. Marty Mayo for providing financial support which allowed me to have a continued focus on my research path. I also want to acknowledge Dr. Bijoy Kundu for helping me every step of the way. I will always be grateful for your advice and friendship.

Finally, I want to acknowledge the following people who helped me in so many valuable ways: Michael Gutneckt, Dr. Amy Bouton, Dr. Yi Zhang, Alexander Yoo, Jake Wamsley, Li Xiao, Jack Roy, Dr. Donfeng Pan, Jim Weirich, Gina Wimer, Dr. Moriel Vandsburger, and Dr. Elizabeth Redente. 


\section{Table of Contents}

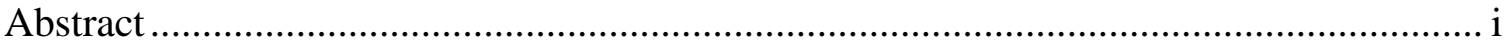

Acknowledgements ............................................................................................ iv

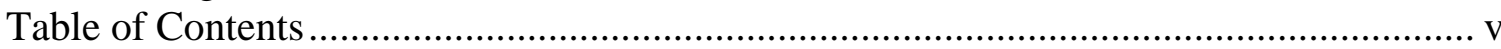

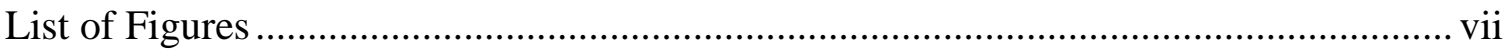

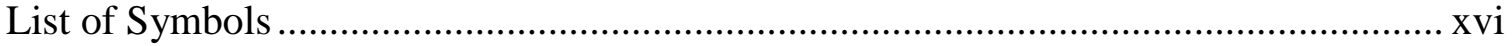

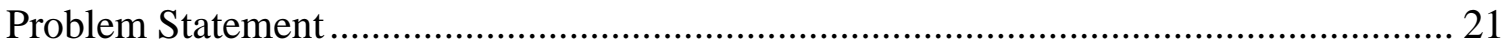

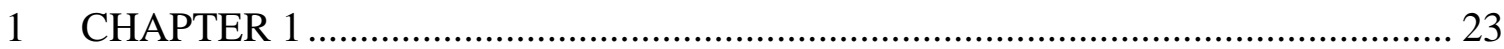

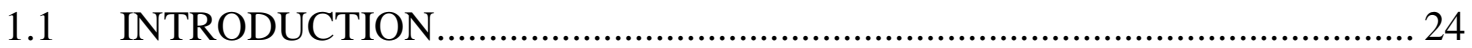

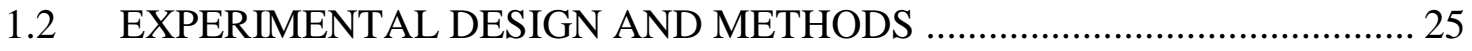

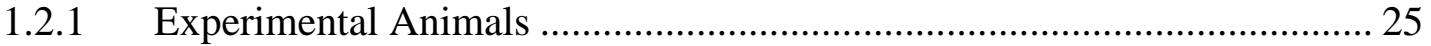

1.2.2 PET and CT Imaging Protocol............................................................... 26

1.2.3 Image Reconstruction .................................................................... 26

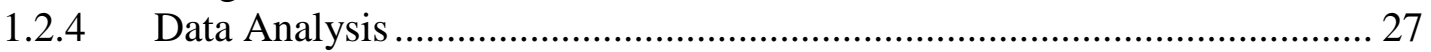

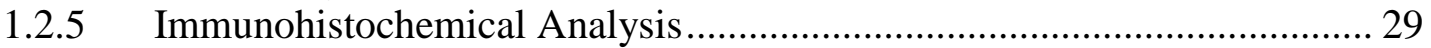

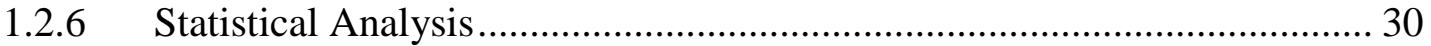

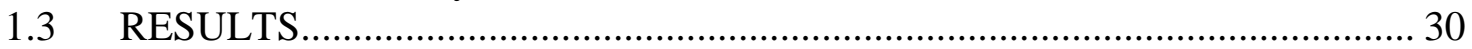

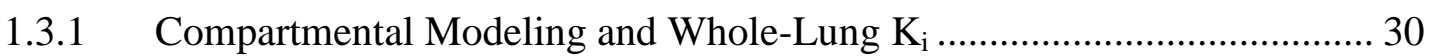

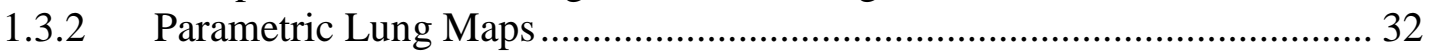

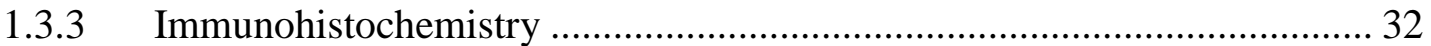

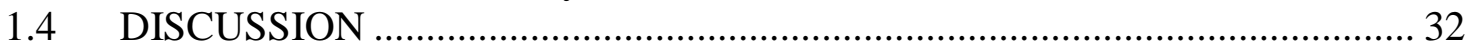

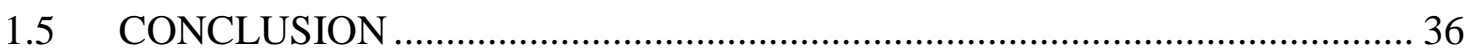

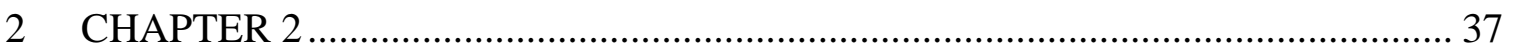

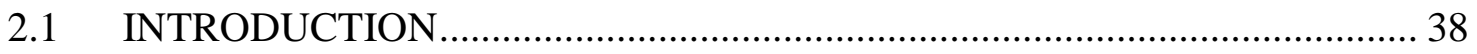

2.2 EXPERIMENTAL DESIGN AND METHODS ......................................... 38

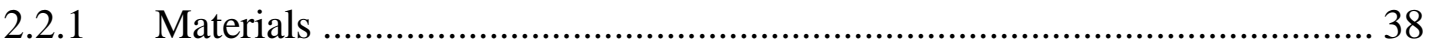

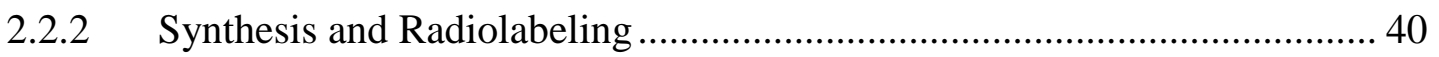

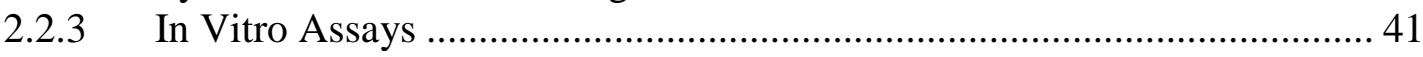

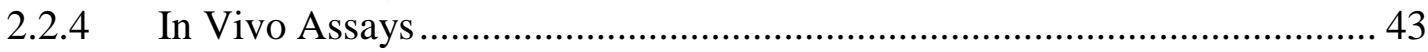

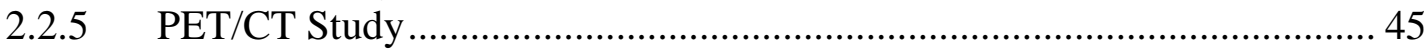

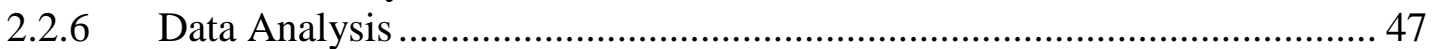

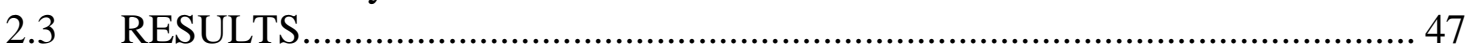

2.3.1 Peptide Synthesis and Radiolabeling ................................................. 47

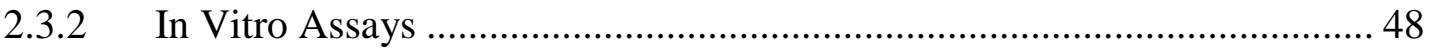

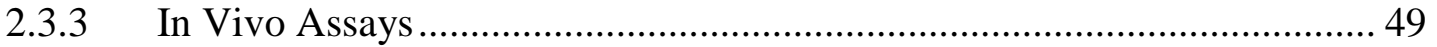

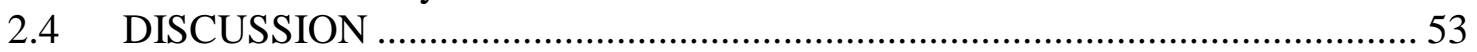

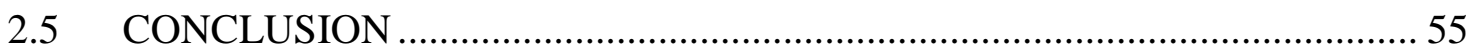

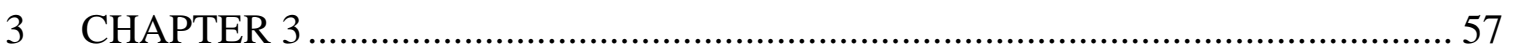

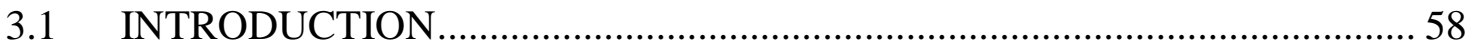

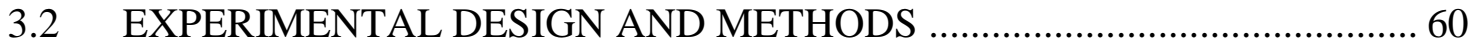

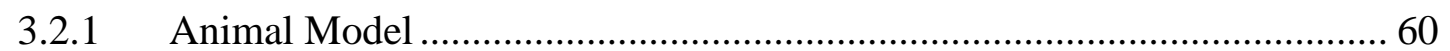

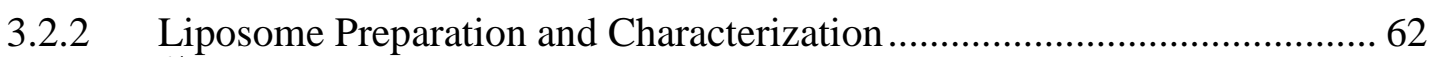

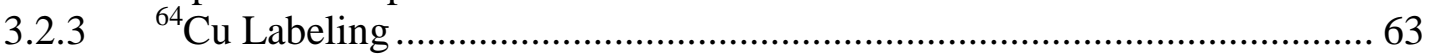

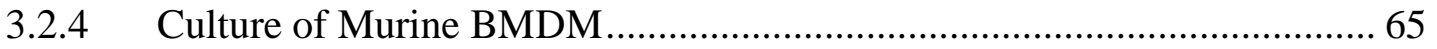

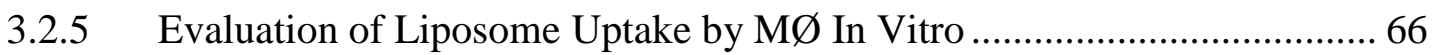


3.2.6 In Vivo Studies of Liposome Distribution and Uptake by MØ ................. 67

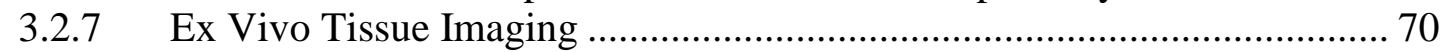

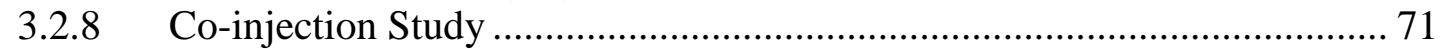

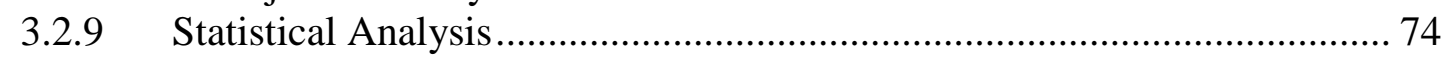

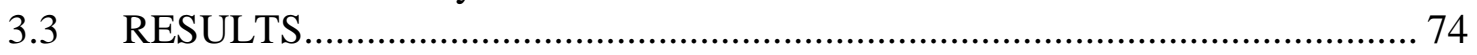

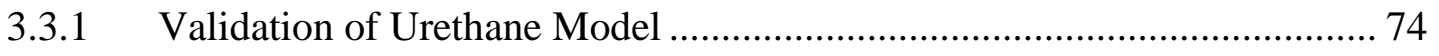

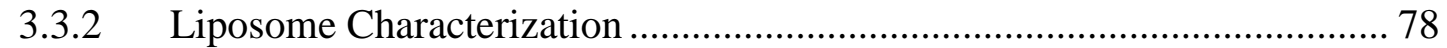

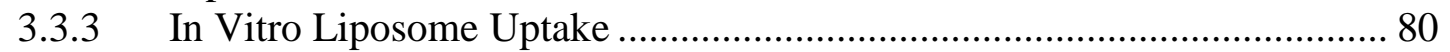

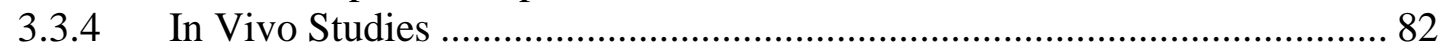

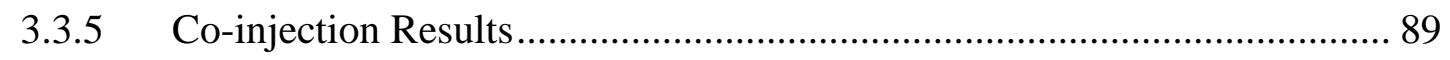

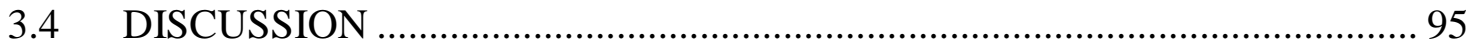

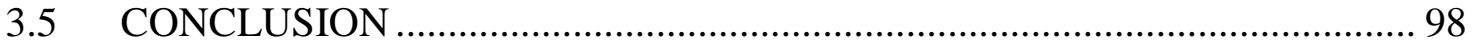

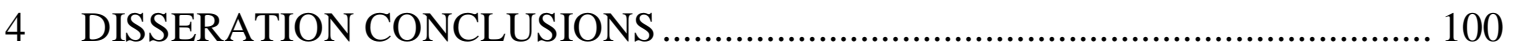

5 FUTURE RESEARCH.............................................................................. 103

6 APPENDIX A: Image-Derived Input Function Validation................................. 107

7 APPENDIX B: Mechanism of Lung Tumor Induction by Urethane ..................... 116

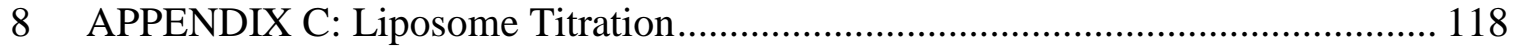

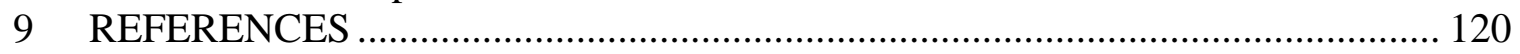




\section{List of Figures}

Figure 1-1. Two-tissue-three-compartment model of FDG kinetics. The $\mathrm{k}_{1}\left(\mathrm{ml} \mathrm{min}^{-1} \mathrm{ml}^{-}\right.$

$\left.{ }^{1}\right), \mathrm{k}_{2}\left(\min ^{-1}\right), \mathrm{k}_{3}\left(\mathrm{~min}^{-1}\right)$, and $\mathrm{k}_{4}\left(\mathrm{~min}^{-1}\right)$ values represent the rate constants for FDG forward transport, reverse transport, phosphorylation, and dephosphorylation, respectively

Figure 1-2. A representative plot showing a lung tissue TAC from a control animal and the associated compartment model fit $\left(\mathrm{R}^{2}=0.92\right)$. Modeling produced good fits to the observed data. 31

Figure 1-3. Whole-lung $\mathrm{K}_{\mathrm{i}}$ values for control and $K$. pneumoniae-challenged mice calculated from three-compartment model analysis (see Data Analysis). The black diamonds represent lung $K_{i}$ values of individual mice while the gray diamonds represent the average of the group. ${ }^{*} P<0.05$ compared with the non-infected control group. ..... 31 Figure 1-4. Parametric $\mathrm{K}_{\mathrm{i}}$ images of segmented lungs of a control mouse and $K$. pneumoniae-challenged mouse computed by Patlak analysis (A). The $\mathrm{K}_{\mathrm{i}}$ value of a single voxel within an area of high FDG uptake (indicated by arrow) is significantly higher than that of the whole-lung $\mathrm{K}_{\mathrm{i}}$ value computed by the Patlak method (B) 33

Figure 1-5. Immunohistochemical staining of neutrophils (stained with a rat anti-mouse IgG) and macrophages (stained with anti MAC-2 IgG) in lung tissue excised from a control and a $K$. pneumoniae infected mouse (42 hr post challenge). Immunostained cells appear dark brown. Control lungs revealed no neutrophils (A) or macrophages (B), only normal alveolar wall structure. Infected lungs had significant neutrophil accumulation (C), with very little macrophage infiltration (D, indicated by arrowheads). (Magnification, x400.) 34 
Figure 2-1. A representative saturation curve of cFLFLF-PEG $-{ }^{64} \mathrm{Cu}$ specifically bound to human neutrophils. Binding affinity was computed to be $17.71 \mathrm{nM}$. Binding data have also been plotted as Scatchard plot. Nonspecific binding was computed in the presence of excess cold compound and is estimated to contribute to $20 \%-30 \%$ of total binding. .... 48

Figure 2-2. Stimulation of the neutrophil oxidative burst by cFLFLF-PEG-Cu and fMLF as detected by luminol-enhanced chemiluminescence. The results are reported as peak chemiluminescence as a percentage of TNF-primed and fMLF-stimulated $\left(10^{-6} \mathrm{M}\right)$ control. cFLFLF-PEG-Cu demonstrated no agonist activity towards neutrophils (EC50 value not computable), while fMLF-stimulated neutrophil superoxide release with an EC50 of $3.7 \times 10^{-7} \mathrm{M}$. Data are reported as mean \pm SEM for at least 2 independent measurements. 49

Figure 2-3. Tissue and organ accumulation of cFLFLF-PEG- $-{ }^{64} \mathrm{Cu}$ at $18 \mathrm{~h}$ after injection in control and $K$. pneumoniae-challenged mice, expressed as \% ID/g of tissue. Mean lung radioactivity is 3.8 times greater in infected lungs than in control lungs $(* P<0.05)$.

Figure 2-4. Examples of CT and PET images of control and K. pneumoniae-challenged mice. PET images were obtained $18 \mathrm{~h}$ after i.v. injection of cFLFLF-PEG- ${ }^{64} \mathrm{Cu}$. Both transverse (top row) and coronal (bottom row) images are shown. In mice administered K. pneumoniae, there is extensive attenuation in lungs on CT scans, compared with controls. PET scans revealed that $K$. pneumoniae-challenged mice had visually more tracer uptake in lung tissue than did controls, as was quantified by ROI analysis. $\mathrm{H}=$ heart; $\mathrm{L}=$ liver. 51 
Figure 2-5. Myeloperoxidase activity in lung homogenates, expressed as change in optical density per minute per milligram of tissue. Myeloperoxidase activity measured in K. pneumoniae-challenged lungs was approximately 6-fold higher than that measured in control lungs $(* P<0.05) . \Delta \mathrm{OD}=$ change in optical density. 52

Figure 2-6. $18 \mathrm{~h}$ following injection of cFLFLF-PEG- ${ }^{64} \mathrm{Cu}$, SUVs measured in $K$. pneumoniae-challenged lungs were 5.8 times greater in than in control lungs $(* P<$ $0.005)$ 53

Figure 2-7. Immunohistochemical staining of neutrophils (stained with a rat anti-mouse IgG [MCA771G; Serotec]) and macrophages (stained with anti MAC-2 IgG [ACL8942P; Accurate]) in lung tissue excised from a control and a $K$. pneumoniae-challenged mouse (42 hr post administration). Immunostained cells appear dark brown. Control lungs revealed no neutrophils (A) or macrophages (B), only normal alveolar wall structure. Infected lungs had significant neutrophil accumulation $(\mathbf{C})$, with very little macrophage infiltration (D, indicated by arrows). (Magnification, x400.) 54

Figure 3-1. A schematic diagram of liposomes. DOTA-containing plain (left) and $\mathrm{Man}_{3}$ (right) liposomes allow for remote loading of the PET imaging agent, ${ }^{64} \mathrm{Cu}$. The mean

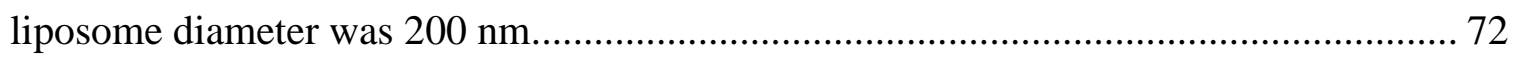

Figure 3-2. A representative photomicrograph of a lung section stained with H\&E 24 weeks after injection of urethane. 75

Figure 3-3. Representative IHC staining of F4/80 in lung tissue from a urethane-treated mouse 24 weeks after treatment. As shown in (A), a moderate number of $\mathrm{F} 4 / 80^{+}$ macrophages have infiltrated the tumor border, with very few localized within the tumor mass. Quantification of IHC images revealed a seven-fold increase in $\mathrm{F} 4 / 80^{+}$cell density 
in the peritumoral compartment compared to histologically normal lung $(* P<0.05)(\mathbf{B})$.

76

Figure 3-4. Macrophage M2 polarization in lungs of a urethane-treated mouse 25 weeks after treatment. The H\&E image shows a tumor boundary $(\mathrm{T})$ with moderate immune cell infiltration in the stroma (A). Immunofluorescence staining of the tumor stroma outlined in the H\&E image by the black dashed box is shown in $(\mathbf{B})$. At the edge of the tumor (indicated by dashed white line), the majority of macrophages identified by F4/80 (red) also stain for CD206 (green) indicating M2 polarization. 77

Figure 3-5. Based on a simple threshold, we estimated that $94 \%$ of F4/80 ${ }^{+}$cells $($Red $)$coexpress the mannose receptor (CD206, green), as indicated by blue circles. Yellow circles indicate $\left(\mathrm{F} 4 / 80^{+}, \mathrm{CD} 206^{-}\right)$cells. This finding is consistent with other studies that have shown significant M2-polarization of TAMs during a similar phase post urethane treatment. 79

Figure 3-6. Con A-induced agglutination assay of $\mathrm{Man}_{3}$ and plain liposomes. $10 \mu \mathrm{L}$ of Con-A (25 $\mu \mathrm{g})$ was added to $100 \mu \mathrm{L}$ of plain (gray triangles) or $\mathrm{Man}_{3}$ (black squares) liposomes and absorbance at $360 \mathrm{~nm}$ was measured as a function of time. The addition of Con-A to $\mathrm{Man}_{3}$-liposomes resulted in a 10-fold increase in solution turbidity, signifying the presence of mannose residues on these liposomes. In contrast, no change in turbidity was observed for plain liposomes. 80

Figure 3-7. An elution profile of ${ }^{64} \mathrm{Cu}$ and $\mathrm{DiO}$ following a $1 \mathrm{~h}$ liposome incubation period with ${ }^{64} \mathrm{Cu}$ and 2-hydroxyquinoline. The profile was constructed by collecting the eluate in $100 \mu \mathrm{L}$ aliquots and measuring the radioactivity and fluorescence. Colocalization of the peaks indicates that ${ }^{64} \mathrm{Cu}$-liposome loading was successful. 81 
Figure 3-8. Expression of CD206 (mannose receptor) measured by flow cytometry (A) and Western blot analysis (B). Flow data represent average of three trials. Error bars indicate standard deviations. ${ }^{*} P<0.05$ vs. IL-4/13-stimulated cells. 82

Figure 3-9. Association of DiO-labeled plain and $\mathrm{Man}_{3}$-liposomes with $\mathrm{M} \emptyset(\mathrm{LPS} / \mathrm{IFN}-\gamma)$ and $M \emptyset(I L-4 / 13)$ measured by flow cytometry. Cells were incubated with either liposome type for $90 \mathrm{~min}$ after which cell-associated DiO fluorescence was measured. (A) Representative fluorescence histograms of MØ(LPS/IFN- $\gamma$ ) and MØ(IL-4/13) following incubation with either plain (black histograms) or $\mathrm{Man}_{3}$ liposomes (red histograms). Non-liposome incubated cells (gray histograms) are shown for reference. (B) Geometric MFI values and standard deviation of DiO fluorescence of all replicates (n

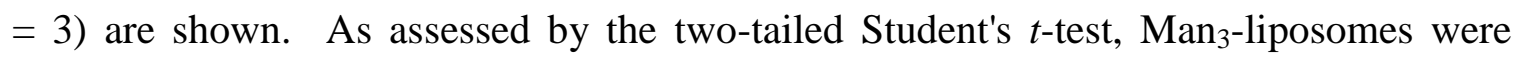
associated with $\mathrm{M} \emptyset(\mathrm{IL}-4 / 13)$ significantly more than M $\varnothing(\mathrm{LPS} / \mathrm{IFN}-\gamma)$. Likewise, compared to plain liposomes, $\mathrm{Man}_{3}$-liposomes were more strongly associated with $\mathrm{M} \varnothing(\mathrm{IL}-4 / 13)(P<0.05$, for both). 83

Figure 3-10. Blood clearance curves of $\mathrm{Man}_{3}$-Liposomes (black curve) and plain Liposomes (gray curve) measured in normal mice are shown. Liposomes were i.v. injected and the blood was withdrawn at different time intervals. Sample radioactivity concentrations were normalized by the injected dose and multiplied by $7.3 \%$ of the body weight to approximate the \%ID in blood as shown in Eq. 1. Values shown are the mean of three experiments. Error bars represent standard deviation of the mean. 84 Figure 3-11. Representative spin echo MR images of a saline (top) and urethane-injected (bottom) mouse 24 weeks following treatment. These scans were acquired approximately 30 min after gadolinium injection. Lungs tumors, as indicated by yellow arrows, are 
clearly visible in the urethane-treated mouse. In contrast, no lung tumors are detected in the saline-treated mouse. 85

Figure 3-12. Representative in vivo images showing a lung tumor on coronal MRI (A) with enhanced ${ }^{64} \mathrm{Cu}$-labeled $\mathrm{Man}_{3}$-liposome uptake on PET $6 \mathrm{~h}$ after i.v. injection (B). PET-MR image registration (C) verifies tumor localization of the PET signal. Ex vivo fluorescence image of the lung was obtained to assess DiO distribution. A photo shows the tumor (D) which exhibited higher DiO accumulation compared to non-tumor lung areas. Ex vivo PET maximum intensity projection image (F) showed focal ${ }^{64} \mathrm{Cu}$ signal in the area of the lung spatially corresponding to the tumor shown in the photograph........ 86

Figure 3-13. In vivo PET quantification of ${ }^{64} \mathrm{Cu}-\mathrm{Man}_{3}$-liposome uptake measured in tumor and lung tissue at $6 \mathrm{~h}$ post injection. MRI-guided ROIs were drawn over tumors and areas in the lungs without visible tumor mass. The radioactivity concentration measured in tumors was corrected for PVE using a look-up table approach. RC-corrected ${ }^{64} \mathrm{Cu}-\mathrm{Man}_{3}$-liposome uptake $(\% \mathrm{ID} / \mu \mathrm{L})$ was $9.6 \pm 2.5$ in tumor lesions compared to $1.4 \pm$ 0.6 in normal lung tissue $(* P<0.05)$. 87

Figure 3-14. Confocal fluorescence microscopy revealed internalization of DiO-labeled $\mathrm{Man}_{3}$-liposomes (green) by $\mathrm{F} 4 / 80^{+}$macrophages (red) within the tumor stroma $6 \mathrm{~h}$ after i.v. liposome injection. Cell nuclei are stained with DAPI (blue). (A) A co-localized confocal image shows the intracellular localization of $\mathrm{Man}_{3}$-liposomes within TAMs. The enlarged view of the cell indicated by the yellow arrow is shown in (B), clearly shows a clustered distribution of liposomes consistent with storage in macrophage endosomal structures. 88 
Figure 3-15. Excitation (A) and emission (B) spectra for each fluorescent dye used for the analysis of liposome uptake by TAMs by confocal microscopy. DiO and DiD are the fluorescent dyes used to label liposomes. DAPI is used to visualize cell nuclei and TRITC is the dye conjugated to the secondary antibody used for staining the anti-mouse F4/80 primary antibody

Figure 3-16. A preliminary well plate study demonstrating that $\mathrm{DiO}$ and $\mathrm{DiD}$ fluorescence associated with different liposome types can be detected separately. The top row shows that DiO-labeled $\mathrm{Man}_{3}$-liposomes could be imaged apart from DiD using an excitation and emission filter pair of $500 \mathrm{~nm}$ and $540 \mathrm{~nm}$, respectively. Likewise, the bottom row shows that DiD-labeled plain liposome could be imaged apart from $\mathrm{DiO}$ using an excitation and emission filter pair of $605 \mathrm{~nm}$ and $640 \mathrm{~nm}$, respectively. This studied confirms that following their co-injection, liposomes can be imaged separately allowing their biodistribution to be uniquely tracked.

Figure 3-17. Representative photos and fluorescent images from the liposome coinjection study. Images of excised lungs $6 \mathrm{~h}$ after the co-injection of $\mathrm{Man}_{3}$ and plain liposomes (A) and $\mathrm{Man}_{3}$ and PEG liposomes (B). Strong fluorescence signal associated with $\mathrm{Man}_{3}$ - and plain liposomes is localized to lung tumors (identified by white arrows on the photo). However, compared to $\mathrm{Man}_{3}$-liposomes, plain liposomes exhibit a higher background signal. PEG liposomes show a diffuse lung distribution and consequently poor tumor contrast likely due to their enhanced blood circulation time. 92

Figure 3-18. Tumor-to-tissue ratios measured from fluorescence images of harvested organs following the co-injection of $\mathrm{Man}_{3}$ and plain liposomes (A) and $\mathrm{Man}_{3}$ and PEG liposomes (B). Man 3 -liposomes exhibited a higher tumor-to-remote lung and tumor-to- 
spleen ratio compared to plain liposomes, while tumor-to-liver ratios were comparable. $\mathrm{Man}_{3}$-liposomes also exhibited a high tumor-to-remote lung ratio following co-injection with PEG liposomes, likely due to the slow rate of blood clearance of PEG liposomes. PEG liposomes also showed a reduced tumor-to-liver ratio, consistent with a lower rate of capture by the RES.

Figure 3-19. Confocal images of lung tumor stroma following liposome co-injection. Following their co-injection, $\mathrm{Man}_{3}$ and plain liposomes, indicated by green and yellow fluorescence, respectively, were both internalized by F4/80 ${ }^{+}$cells (A). Similarly, Man $3^{-}$ and PEG liposomes were also internalized by $\mathrm{F} 4 / 80^{+}$cells following their co-injection (B). The spatial distribution of each dye within a given $\mathrm{F} 4 / 80^{+}$cell appears to be identical, suggesting entry into cells by common surface receptors 94

Figure 6-1. Transaxial view of attenuation-corrected phantom images reconstructed with a) FBP and b) MAP and the recovery coefficient curves generated from them (c) ...... 110 Figure 6-2. Transverse FDG PET images of a mouse heart reconstructed using a OSEMMAP with cardiac gating, b OSEM-MAP without cardiac gating, $\mathbf{c}$ FBP with cardiac gating, and $\mathbf{d}$ FBP without gating. Common line profiles through the LVBP are shown below each image. Only cardiac-gated, OSEM-MAP reconstructions produced images that allowed accurate measurement of blood radioactivity concentration in the LVBP. 111 Figure 6-3. Representative IDIFs, derived from a common LVBP ROI drawn on PET images shown in panels (a), (c), and (d) of the previous figure are shown. The square and circular boxes, which represent manually sampled blood activity concentrations taken at 43 and 56 minutes respectively, are only in close agreement with activity concentrations of the IDIF derived from the OSEM-MAP reconstructed, cardiac-gated 
image (solid gray line). The IDIF derived from the non-gated, FBP reconstructed image (black dotted line) suffered the highest amount of spillover contamination. 113

Figure 6-4. Bland-Altman plot showing the agreement between image-derived (corrected for attenuation and PV effects) and physically sampled blood radioactivity concentrations in five mice. The mean difference was $-0.06 \mu \mathrm{Ci} / \mathrm{mL}$ (solid line) and the lower and upper limits of agreement (dashed lines) were -0.22 and 0.34 , respectively. 114 Figure 8-1. Dose dependent increases in M2 macrophage uptake of DiO-labeled plain (๘) and mannosylated (») liposomes. The uptake of DiO-labeled liposomes was calculated as the geometric mean of fluorescence in the green channel after the background fluorescence was subtracted 


\section{List of Symbols}

${ }^{64} \mathrm{Cu}-$ Copper-64

$\mathrm{AC}$ - Adenocarcinoma

$\mathrm{B}_{\max }-$ Maximum Binding

BM - Bone Marrow

$\mathrm{Bq}-$ Becquerel

BSA - Bovine Serum Albumin

BV - Blood Volume Component

BW - Body Weight

$\mathrm{C}_{\mathrm{b}}(\mathrm{t})$ - Time-Course of Tracer Concentration in the Blood

$\mathrm{C}_{\mathrm{t}}(\mathrm{t})$ - Time-Course of Tracer Concentration in Tissue

cFLFLFK - N-cinnamoyl-phe-(D)-Leu-phe-(D)-Leu-phe-lys

cFLFLFKCONH 2 - N-cinnamoyl-phe-(D)-Leu-phe-(D)-Leu-phe-lys-CONH ${ }_{2}$

CLIO-gly - Dextran-Coated Magnetofluorescent Nanoparticle Decorated with the Amino Acid Glycine

Con-A - Concanavalin A

$\mathrm{CoV}$ - Coefficient of Variance

CT - Computed Tomography

DAPI - 4',6-diamidino-2-phenylindole

DiD - 1,1'-Dioctadecyl-3,3,3',3'-Tetramethylindodicarbocyanine

DiO - 3,3'-Dioctadecyloxacarbocyanine Perchlorate

DOTA - 1,4,7,10-tetra-azacyclododecane-1,4,7,10-tetraacetic acid

DPPE - Dipalmitoylphophatidylethanolamine 
DTPA - DiethyleneTriaminePentaacetic Acid

EC - Ethyl Carbamate

EC-50 - Median Effective Concentration

EDTA - Ethylenediaminetetraacetic Acid

FBP - Filtered Black Projection

FDG - 2'-deoxy-2'-[ $\left[{ }^{18}\right.$ F]fluoro-D-glucose

fMLF - N-Formyl-Methionine-Leucine-Phenylalanine

FMT - Fluorescence Molecular Tomography

FPR - Formyl Peptide Receptor

FWHM - Full-Width-at-Half-Maximum

$\mathrm{g}_{\text {sample }}$ - Mass of Blood Sample

H\&E - Hematoxylin and Eosin

HA - Hepatic Arterial

HEPES - 4-(2-Hydroxyethyl)-1-Piperazine-Ethanesulfonic Acid

HPLC - High-Performance Liquid Chromatography

HTS - High Throughput Screening

ID - Injected Dose

IDIF - Image-Derived Input Function

IFN- $\gamma-$ Interferon- $\gamma$

IgG - Immunoglobulan G

IHC - Immunohistochemistry

IL - Interleukin

i.p. - Intraperitoneal 
K. pneumoniae - Klebsiella pneumoniae

$\mathrm{k}_{1}$ - Rate Constant for FDG forward transport

$\mathrm{k}_{2}$ - Rate Constant for FDG Reverse Transport

$\mathrm{k}_{3}$ - Rate Constant for FDG Phosphorylation

$\mathrm{k}_{4}$ - Rate Constant for FDG Dephosphorylation

$\mathrm{K}_{\mathrm{d}}-$ Equilibrium Dissociation Constant

$\mathrm{K}_{\mathrm{i}}-$ Net Influx Constant

LPS - Lipopolysaccharide

LV - Left Ventricle

LVBP - Left Ventricle Blood Pool

M-CSF - Macrophage-Colony Stimulating Factor

MALDI-TOF - Matrix-Assisted Laser Desorption/Ionization Time-of-Flight

$\mathrm{Man}_{3}$ - Mannotriose

$\mathrm{Man}_{3}$-liposomes - Mannosylated DOTA Encapsulated Liposomes

MFI - Mean Fluorescence Intensity

MMP-9 - Matrix Metalloproteinase 9

MRI - Magnetic Resonance Imaging

MØ - Macrophage

OCT - Optimal Cutting Temperature

OD - Optical Density

OSEM-MAP - Ordered-Subset Expectation Maximization-Maximum A Posteriori

$\mathrm{p} / \mathrm{s}-$ Photons/Second

PS - Phosphatidylserine 
PBS - Phosphate Buffered Saline

PC - Phosphatidylcholine

PEG - Polyethyleneglycol

PET - Positron Emission Tomography

PG-Gd-NIR813 - poly(1-glutamic acid)-Gd-chelated p-aminobenzyl-

diethylenetriaminepentaacetic acid

PV - Partial Volume

PVE - Partial Volume Effect

$\mathrm{RC}-$ Recovery Coefficient

RES - Reticuloendothelial System

ROI - Region of Interest

RF - Radio-Frequency

$\mathrm{RP}$ - Reversed-Phase

SEC - Size Exclusion Tomography

SUV - Standardized Uptake Value

$t$ - Time

$t$-Boc-PEG-NHS - $t$-Butoxycarbonyl-Protected PEG-Succinimidyl Ester

TAC - Time-Activity Curve

TAM - Tumor-Associated Macrophage

TAN - Tumor-Associated Neutrophil

TME - Tumor Microenvironment

TE - Echo Time

TNF- $\alpha$ - Tumor Necrosis Factor- $\alpha$ 
TR - Repetition Time

TRITC - TetramethylRhodamineIso-Thiocyante

TSB - Trypticase Soy Broth

VC - Vinyl Carbamate

VEGF - Vascular Endothelial Growth Factor

$\alpha-\mathrm{MEM}-\alpha$-Minimum Essential Medium

$\mu \mathrm{Ci} i_{I D}-$ Radioactivity of Injected Dose

$\mu \mathrm{Ci}_{\text {sample }}-$ Radioactivity Measured in the Blood Sample

$\%$ ID - Injected Activity

$\% \mathrm{ID}_{\mathrm{TBM}}-$ Percentage of the Injected Dose in Total Body Mass 


\section{Problem Statement}

The pulmonary inflammatory response is a highly coordinated multicellular process geared towards combating infection and injury. This response, which involves the activation and mobilization of a variety of cell types from the circulation to the site of injury, ultimately promotes the repair process. Although it is critical to survival, an inflammatory response that becomes dysregulated can lead to persistent accumulation and activation of leukocytes, which is believed to be a key factor in the pathogenesis of a wide variety of diseases that affect the airways of the lungs. Neutrophils, a class of white blood cells, are often recruited to sites of infection. Because the presence of neutrophils and their secretory markers have been strongly linked to the pathogenesis of a number of important disease processes such as COPD and cystic fibrosis[1-4], there has been great effort aimed at understanding the role and behavior of these cells in the context of debilitating lung diseases.

For the majority of pulmonary diseases, the ability to image the neutrophil response in the lung is an important goal to better understand cellular mechanisms that contribute to normal and disease states as well as for assessing future anti-inflammatory therapies. However, the lung parenchyma is not a site which is easily accessible, making the quantification and monitoring of inflammatory cell behavior a significant challenge. Traditional imaging approaches for evaluating pulmonary function or anatomy do little to detect or quantify the inflammatory component. Current clinical procedures for assessing neutrophilic lung inflammation include collecting cells by bronchoalveolar lavage, obtaining lung biopsies, and tracking neutrophils previously labeled with a Single Photon Emission Computed Tomography (SPECT) [5, 6] or Positron Emission Tomography 
(PET) [7] radioisotope following intravenous injection. However, each of these procedures possesses significant limitations and none possess all of the desirable characteristics of being noninvasive, easily repeatable, and able to assess neutrophil distribution and activity on a whole-lung level. For this dissertation I explored two different noninvasive, molecular imaging strategies for monitoring lung neutrophilic inflammation based on PET imaging in mice. Chapter 1 explores the use of 2'-deoxy-2'$\left[{ }^{18}\right.$ F]fluoro-D-glucose (FDG)-PET with compartmental model based on an image-derived blood input function for assessing neutrophil metabolic rate of glucose uptake: a surrogate marker for neutrophil activation. Chapter 2 explores a copper-64 $\left({ }^{64} \mathrm{Cu}\right)$ labeled peptide for targeting surface receptors over-expressed on infiltrated neutrophils.

Macrophages are another leukocyte that play a critical role in the pathophysiology of many pulmonary diseases. Macrophage numbers are markedly increased in the lung and alveolar space of patients with COPD [8, 9] and CF [10, 11]. Furthermore, recent evidence has emerged strongly linking macrophages of the M2 subtype to lung tumor progression in preclinical as well as in human studies based on immunohistochemical analyses [12-15]. Therefore, the ability to effectively and specifically label macrophages in the lungs using a targeted nanoparticle agent could facilitate preclinical studies investigating the role of these cells in pulmonary disease progression over time as well as monitoring the effects of new anti-macrophage therapies. Our approach, described in Chapter 3, is to surface coat liposomes with a carbohydrate ligand and compare their uptake to plain liposomes by macrophages in vitro and in vivo. 


\section{CHAPTER 1}

\section{The Application of FDG-PET for Quantifying}

Neutrophil Metabolic Activity in the Lung 


\subsection{INTRODUCTION}

Despite numerous studies that have identified neutrophils as the most abundant inflammatory cell type present in the airways and lung parenchyma of patients with COPD and CF, we currently lack reliable imaging probes capable of monitoring the presence and activity of these cells in situ. Current techniques used in the preclinical setting include bronchoalveolar lavage (BAL), which is a terminal procedure allowing sampling of cells in the airway, and imaging of neutrophils radiolabeled with ${ }^{111}$ In or ${ }^{99 \mathrm{~m}} \mathrm{Tc}$ and monitored by gamma-scintigraphy $[16,17]$. In general, techniques using in vitro labeling of white blood cells suffer the disadvantage of lengthy, laborious, and potentially hazardous labeling procedures. Additionally, such procedures may induce neutrophil activation which may consequently alter their biodistribution and function upon administration. PET imaging of the radioactive glucose analog FDG is a valuable diagnostic procedure in clinical oncology, yet is being increasingly investigated as a probe for neutrophil metabolic activity in the lung [1, 18-21]. FDG is well-suited for tracking neutrophil metabolic activation due to the heightened glucose consumption exhibited by neutrophils when undergoing reactive oxygen species production [22-24]. This elevated demand for glucose forms the basis for probing neutrophil activation by FDG-PET. Prior in vivo animal studies have demonstrated the uptake of FDG by neutrophils in models ranging from pneumonia $[21,25,26]$ to pancreatitis-induced lung injury [23] to sepsis-induced lung injury [24].

Computing the net uptake rate of FDG by three-compartmental model analysis is the gold standard technique for quantitative measurement of glucose utilization by FDGPET [21, 27]. The major disadvantage of this technique is the requirement for 
measurement of the blood input function which is defined as the serial measurement of FDG activity concentration in the blood over the entire scan duration. It is a significant challenge to obtain the input function in mice due to the limited blood volume and the required surgical insertion of an arterial catheter. There have been a variety of approaches taken to obtain the input function in mice including invasive arterial sampling, noninvasive estimation from dynamic PET images, and various combinations thereof [28-35]. The purpose of this study is to demonstrate that the blood input function can be accurately and noninvasively measured from PET images. Finally, using a mouse model of acute pulmonary inflammation, we evaluated the feasibility and accuracy of measuring neutrophil metabolic activity with FDG-PET by three-compartment model analysis with an image-derived input function (IDIF).

\subsection{EXPERIMENTAL DESIGN AND METHODS}

\subsubsection{Experimental Animals}

Twelve male C57BL/6 mice (8-10 weeks old) were used in this study. The induction of bacterial pneumonia is based on a previously reported protocol [36]. Briefly, Klebsiella pneumoniae (strain 43816, serotype 2) was grown overnight and then subcultured for 2 hours to log phase growth. After rinsing, bacteria were diluted in sterile normal saline for inoculation. Six mice were inoculated by oropharyngeal aspiration of $50 \mu \mathrm{L}$ of bacterial suspension (approximately $3 \times 10^{5}$ colony-forming units) under light inhalational anesthesia. Mice showed signs of moderate illness 18-36 hr after inoculation, when imaging was performed. Non-infected (saline-injected) mice $(n=6)$ served as controls. 


\subsubsection{PET and CT Imaging Protocol}

Imaging was performed $24 \mathrm{hr}$ following bacterial (or saline) induction. Following the insertion of a tail-vein catheter, ECG surface electrodes (Blue Sensor, Ambu Inc., Glen Burnie, MD) were placed on both forepaws and the left hindpaw. Anesthetized (1$2 \%$ isoflurane in oxygen) mice were placed in the prone position on a custom designed portable tray designed to reproducibly mount on both the CT and PET scanners, facilitating accurate image co-registration. Sixty-minute dynamic PET scans were performed. Data acquisition was initiated a few seconds before the injection of 18-20 MBq (486-540 $\mu \mathrm{Ci})$ FDG via the catheter. FDG was administered over a span of about 30 seconds and the catheter line was immediately flushed with $100 \mu \mathrm{L}$ of saline to clear the line and maximize FDG activity administered to the mouse. All mice were fasted $4 \mathrm{~h}$ prior to FDG administration.

Physiological monitoring was performed with a Small Animal Instruments, Inc., model 1025L for PET (SAII, Inc., Stony Brook, NY). This device was used for monitoring heart rate, respiration, and core body temperature (rectal probe) and also to generate time-stamped signals for each positron annihilation event marking its location within the cardiac cycle which allows retrospective reordering of the PET data into heartcycle based time-bins. Following the PET scan, mice were immediately transported to the CT scanner [37], where a 10-minute whole-body CT scan was acquired to assist in guiding lung ROI boundaries on fused PET images and correcting the PET data for photon attenuation [38].

\subsubsection{Image Reconstruction}

PET images were reconstructed from list mode data which were divided into 23 
dynamic frames: eleven $8 \mathrm{~s}$ frames, one $12 \mathrm{~s}$ frame, two 1 minute frames, one $3 \mathrm{~min}$ frame, and eight 6 min frames. Each frame was further divided into three segments each representing a unique portion of the cardiac cycle. All PET images were reconstructed using Ordered-Subset Expectation Maximization (OSEM) algorithm with 2 iterations and 12 subsets followed by Maximum A Posteriori (MAP) algorithm (18 iterations). The reconstructed image was composed of 95 axial slices of thickness $0.79 \mathrm{~mm}$ with an inplane voxel dimension of $0.4 \mathrm{~mm} \times 0.4 \mathrm{~mm}(128 \times 128$ pixels $)$.

CT images were reconstructed with a 3D filtered back-projection algorithm using COBRA software (Exxim, Inc., Pleasanton, CA). The CT reconstructed pixel size was $0.15 \mathrm{~mm} \times 0.15 \mathrm{~mm} \times 0.15 \mathrm{~mm}$ on a $320 \times 320 \times 384$ image matrix, but was downsampled to match the spatial resolution of PET upon image co-registration. CT-based attenuation correction was performed using the PET scanner software (microPET Manager, Siemens Preclinical Solutions).

\subsubsection{Data Analysis}

Whole-lung tissue time-activity curves (TACs) were measured from CT-drawn lung ROIs which were transferred to co-registered PET images. In order to cover the entire lung field, all transverse slices containing lungs were analyzed for each mouse. IDIFs were measured by manually drawing small circular ROIs on contiguous transverse planes of the PET image in the area corresponding to the left ventricle (LV) blood pool when the cardiac phase was near end-diastole. These ROIs were copied to all dynamic frames and the process was repeated on two to three transverse slices in which the LV cavity was visible. An average blood TAC was computed from each slice. To correct for partial volume effects in the LV blood pool, the average blood TAC was boosted by a 
pre-determined recovery coefficient of 0.92 [39] to yield the IDIF. See Appendix A for additional details on the derivation and accuracy of the IDIF.

The net rate of FDG uptake $\left(\mathrm{K}_{\mathrm{i}}\right)$ in lung tissue, which is linearly related to the local glucose metabolic rate, was determined by fitting experimental PET-derived timeactivity data to a two-tissue, three-compartment model (Figure 1-1) [40, 41]. This model was created to mathematically describe the movement and trapping of FDG in tissue. Using nonlinear regression, the time-course of tracer within the tissue $\left(\mathrm{C}_{\mathrm{t}}(\mathrm{t})\right)$ and the concurrent tracer concentration in the blood $\left(\mathrm{C}_{\mathrm{b}}(\mathrm{t})\right)$ was fitted to the model solution:

$$
\mathrm{C}_{\mathrm{t}}(\mathrm{t})=\frac{\mathrm{k}_{1}}{\alpha_{1}-\alpha_{2}} \times\left[\left(\mathrm{k}_{3}+\mathrm{k}_{4}-\alpha_{1}\right) \mathrm{e}^{-\alpha_{1} \mathrm{t}}+\left(\alpha_{2}-\mathrm{k}_{3}-\mathrm{k}_{4}\right) \mathrm{e}^{-\alpha_{2} \mathrm{t}}\right] \otimes \mathrm{C}_{\mathrm{b}}(\mathrm{t})+\mathrm{BV} \times \mathrm{C}_{\mathrm{b}}(\mathrm{t}) \quad \text { Eq. } 1
$$

where

$$
\alpha_{2,1}=\frac{1}{2}\left(k_{2}+k_{3}+k_{4} \pm \sqrt{\left.\left(k_{2}+k_{3}+k_{4}\right)^{2}-4 k_{2} k_{4}\right)} \text { Eq. } 2\right.
$$

$\otimes$ is the convolution operator, BV is the estimated blood volume component, $t$ is time, and $\mathrm{k}_{1}\left(\mathrm{~mL} \mathrm{~g}^{-1} \min ^{-1}\right), \mathrm{k}_{2}\left(\min ^{-1}\right), \mathrm{k}_{3}\left(\min ^{-1}\right)$, and $\mathrm{k}_{4}\left(\min ^{-1}\right)$ values represent the rate constants for FDG forward transport, reverse transport, phosphorylation, and dephosphorylation, respectively. The net rate of glucose uptake in the tissue of interest, also known as the net influx constant $\left(\mathrm{K}_{\mathrm{i}}\right)$, was computed from the individual rate constant parameters as follows:

$$
\mathrm{K}_{\mathrm{i}}\left(\mathrm{mL} \text { blood } \times \mathrm{mL} \text { tissue } \mathrm{e}^{-1} \times \min ^{-1}\right)=\mathrm{k}_{1} \frac{\mathrm{k}_{3}}{\mathrm{k}_{2}+\mathrm{k}_{3}} \quad \text { Eq. } 3 .
$$




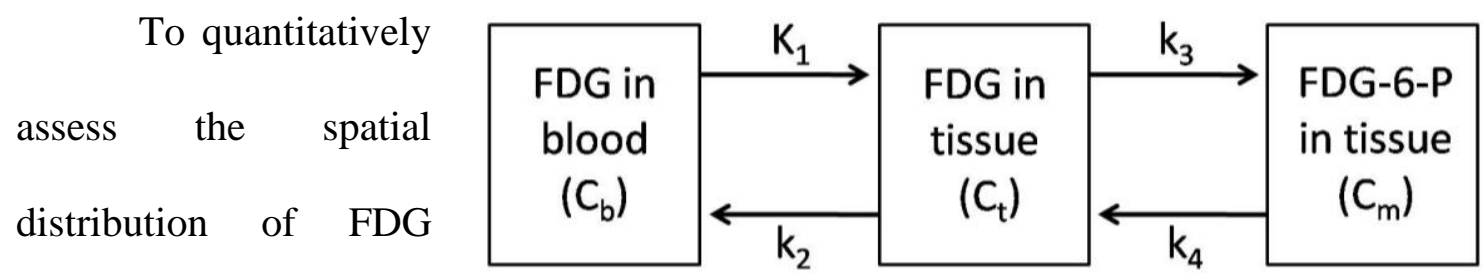

uptake within the lungs,

Figure 1-1. Two-tissue-three-compartment model of FDG kinetics. The $\mathrm{k}_{1}\left(\mathrm{ml} \mathrm{min}^{-1} \mathrm{ml}^{-1}\right), \mathrm{k}_{2}\left(\mathrm{~min}^{-1}\right), \mathrm{k}_{3}\left(\min ^{-1}\right)$, and selective slices from $\mathrm{k}_{4}\left(\mathrm{~min}^{-1}\right)$ values represent the rate constants for FDG forward transport, reverse transport, phosphorylation, and PET images were dephosphorylation, respectively.

transformed into parametric images of local $\mathrm{K}_{\mathrm{i}}$. Voxel-wise $\mathrm{K}_{\mathrm{i}}$ values were computed by Patlak analysis, which is based on the assumption that the ratio of tracer concentration in tissue to that in blood increases linearly when plotted as a function of the integrated blood radioactivity concentration normalized to the blood radioactivity measured at the midpoint of each frame [42]. To estimate $\mathrm{K}_{\mathrm{i}}$, linear regression was performed beginning at 15 minutes post FDG injection to allow time for tracer equilibration between the blood and tissue compartments.

\subsubsection{Immunohistochemical Analysis}

Immunohistochemical analysis was performed on harvested lung tissue to assess the relative distribution of neutrophils compared to macrophages in the lungs of control versus $K$. pneumoniae-challenged mice ( $25 \mathrm{~h}$ post administration). Prior to lung removal, the pulmonary circulation was flushed with saline via the right ventricle to eliminate nonadherent white blood cells. Lungs were then inflated with formalin to distend the alveolar spaces uniformly. The trachea was cannulated and 10\% phosphate-buffered formalin infused at a pressure of $25 \mathrm{~cm} \mathrm{H}_{2} 0$. After fixation, the lung was dissected coronally in the plane of the mainstem bronchus. Adjacent histological sections $(3 \mu \mathrm{m}$ thick) were specifically stained for either neutrophils with a monoclonal rat anti-mouse 
neutrophil IgG (MCA771G; Serotec) or for macrophages with anti MAC-2 IgG (ACL8942P; Accurate). Stained tissues were observed under a Microphot-FXA microscope (Tokyo, Japan).

\subsubsection{Statistical Analysis}

Group data are expressed as the mean \pm standard deviation. Student's $t$-test was used to compare results among groups. Statistical significance was set at $P<0.05$. Sigma-Stat v2.0 (SPSS, Inc, Chicago, IL) was used for statistical testing.

\subsection{RESULTS}

\subsubsection{Compartmental Modeling and Whole-Lung $K_{i}$}

An example of a lung TAC and the associated compartmental model fit from a control animal is shown in Figure 1-2. This figure demonstrates that the model output fits the experimental lung data well. Whole-lung FDG influx constant $\left(\mathrm{K}_{\mathrm{i}}\right)$ was significantly higher in the group of mice administered $K$. pneumoniae compared to controls. The mean whole-lung $\mathrm{K}_{\mathrm{i}}$ for control mice was $(5.1 \pm 1.2) \times 10^{-3}$ vs. $(11.4 \pm 2.0)$ $\times 10^{-3} \min ^{-1}$ for the $K$. pneumoniae-challenged mice $(P=0.0003)$ shown as gray diamonds in Figure 1-3. Within each group, individual whole-lung lung $\mathrm{K}_{\mathrm{i}}$ values are shown (black diamonds). 


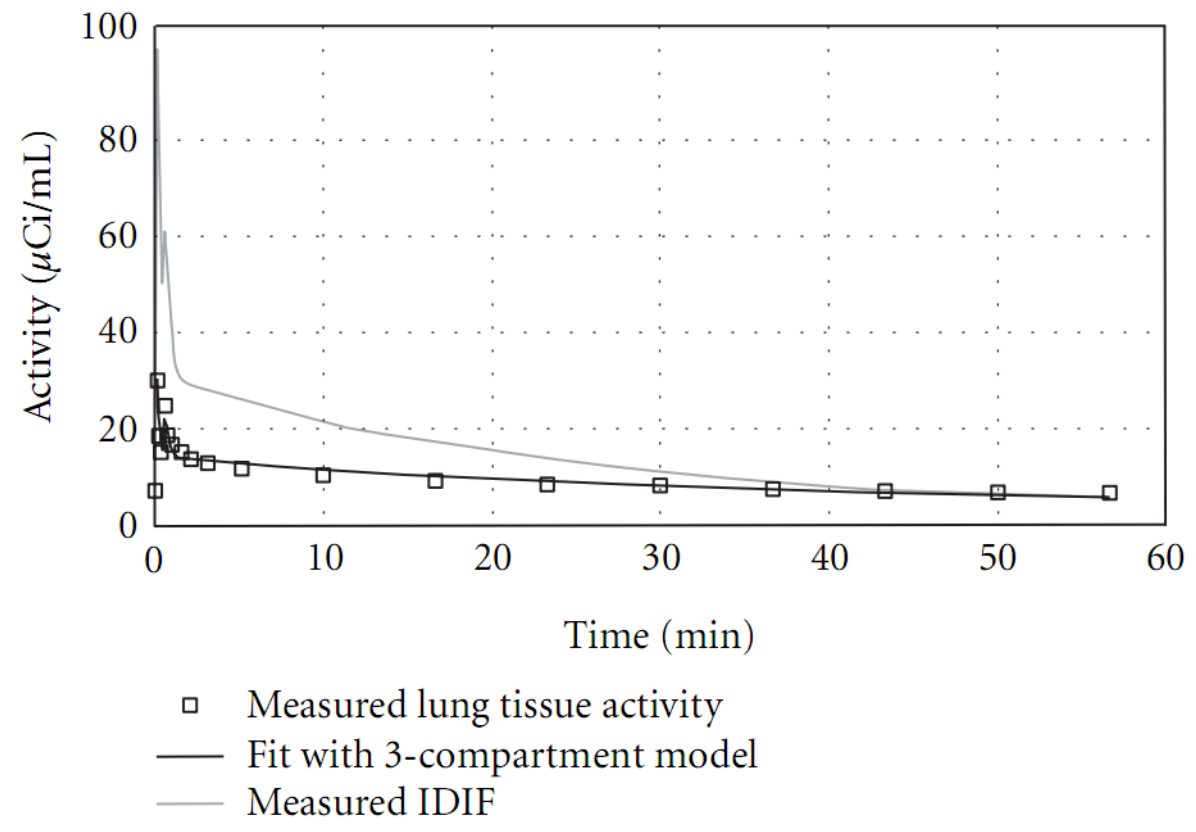

Figure 1-2. A representative plot showing a lung tissue TAC from a control animal and the associated compartment model fit $\left(\mathrm{R}^{2}=0.92\right)$. Modeling produced good fits to the observed data.

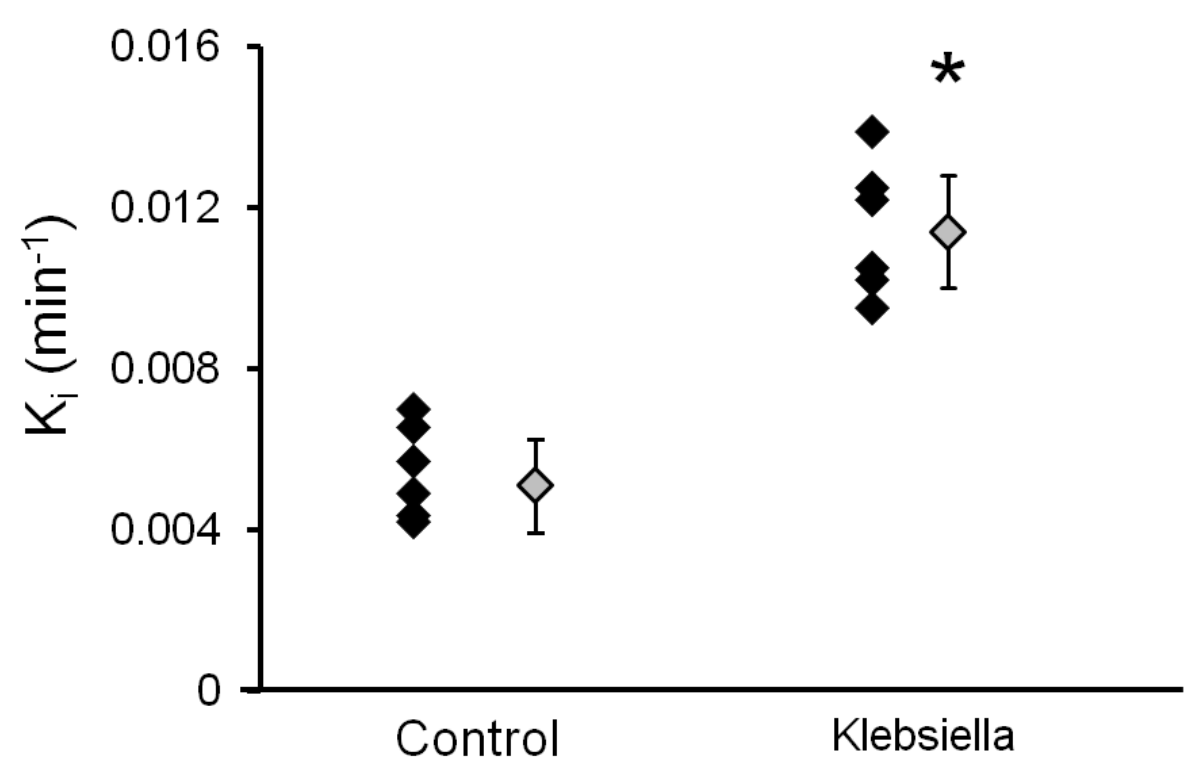

Figure 1-3. Whole-lung $\mathrm{K}_{\mathrm{i}}$ values for control and K. pneumoniae-challenged mice calculated from three-compartment model analysis (see Data Analysis). The black diamonds represent lung $K_{\mathrm{i}}$ values of individual mice while the gray diamonds represent the average of the group. ${ }^{*} P<0.05$ compared with the non-infected control group. 


\subsubsection{Parametric Lung Maps}

Representative parametric $K_{i}$ maps of lungs from a control mouse and a $K$. pneumoniae-challenged mouse are shown in Figure 1-4A. Voxel-wise $\mathrm{K}_{\mathrm{i}}$ values were computed by Patlak analysis using the image-derived blood input function. Segmented lung boundaries were identified and manually outlined on co-registered CT images. Hyper-intense regions are clearly visualized on the $\mathrm{K}_{\mathrm{i}}$ parametric maps of the $K$. pneumoniae infected mice. The K. pneumoniae-challenged mouse clearly demonstrates the spatially heterogeneous nature of the lung disease model. Figure 1-4B shows the result of Patlak analysis performed on a single voxel (indicate by arrow) as well as all lung field voxels of a $K$. pneumoniae-challenged mouse. This comparison serves to highlight the dramatic spatial heterogeneity in lung FDG uptake that can occur after $K$. pneumonia administration, which may not be fully appreciated when whole-lung $\mathrm{K}_{\mathrm{i}}$ values are computed.

\subsubsection{Immunohistochemistry}

Immunohistochemical staining of lung tissue from a control mouse revealed normal alveolar wall structure with very few infiltrated neutrophils (Figure 1-5A) or macrophages (Figure 1-5B). In contrast, the $K$. pneumoniae infected mouse (euthanized 25 h post $K$. pneumoniae challenge) had significant lung neutrophil burden (Figure 1-5C), with very little macrophage infiltration (Figure 1-5D), as indicated by arrowheads.

\subsection{DISCUSSION}

In this work we established a method to quantitatively assess pulmonary FDG uptake in mice by compartmental modeling using an IDIF (see Appendix A for details on the IDIF). Because many lung diseases can be spatially heterogeneous, this assessment 
A
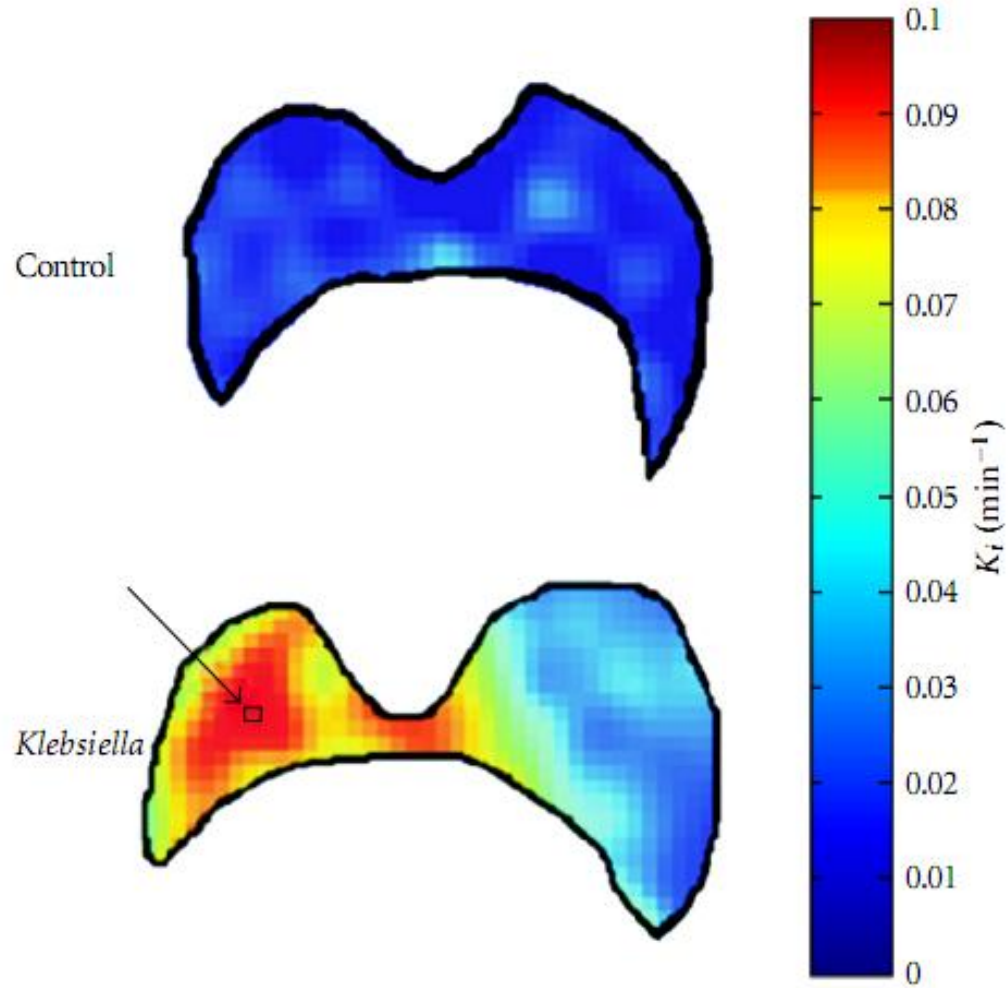

B
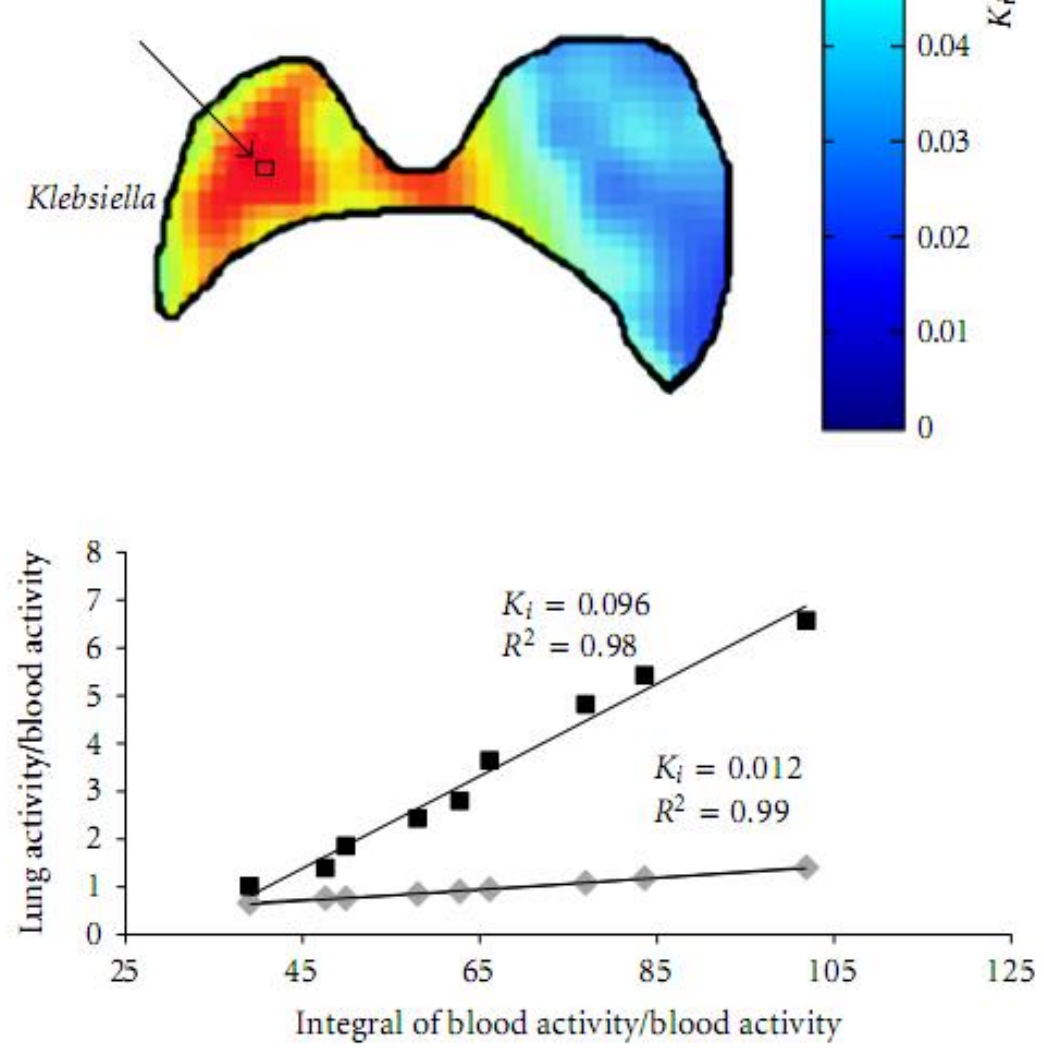

- Single voxel

- Whole lung

Figure 1-4. Parametric $\mathrm{K}_{\mathrm{i}}$ images of segmented lungs of a control mouse and K. pneumoniae-challenged mouse computed by Patlak analysis (A). The $\mathrm{K}_{\mathrm{i}}$ value of a single voxel within an area of high FDG uptake (indicated by arrow) is significantly higher than that of the whole-lung $\mathrm{K}_{\mathrm{i}}$ value computed by the Patlak method $(\mathbf{B})$. 
was made at both the regional level (whole-lung $\mathrm{K}_{\mathrm{i}}$ ) and voxel level. The drawback of voxel-wise analysis is the increased amount of noise when measuring a TAC from a single voxel compared to a TAC measured from all the voxels in the lung field. This increase in noise may lead to inaccuracies in the numerical identification of the kinetic parameters being estimated when fitting data to a compartmental model. We found that Patlak analysis to be better suited for estimating $\mathrm{K}_{\mathrm{i}}$ at the voxel level because it relies on a simple graphical transformation of the time-activity data to directly compute $\mathrm{K}_{\mathrm{i}}$.

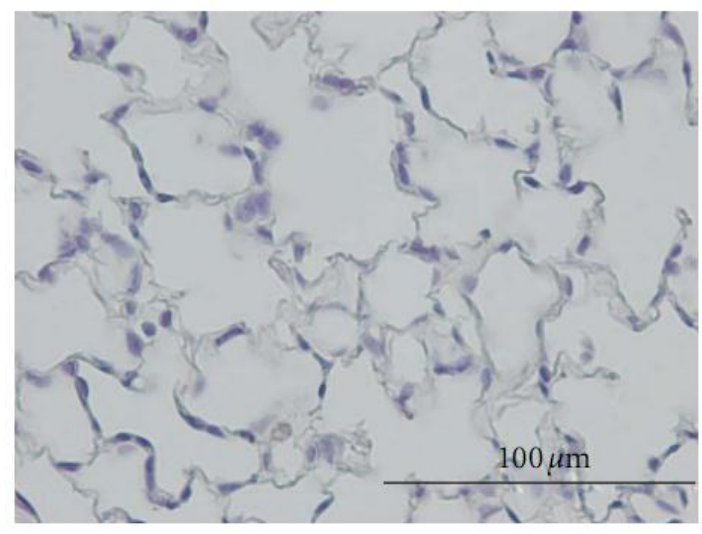

A

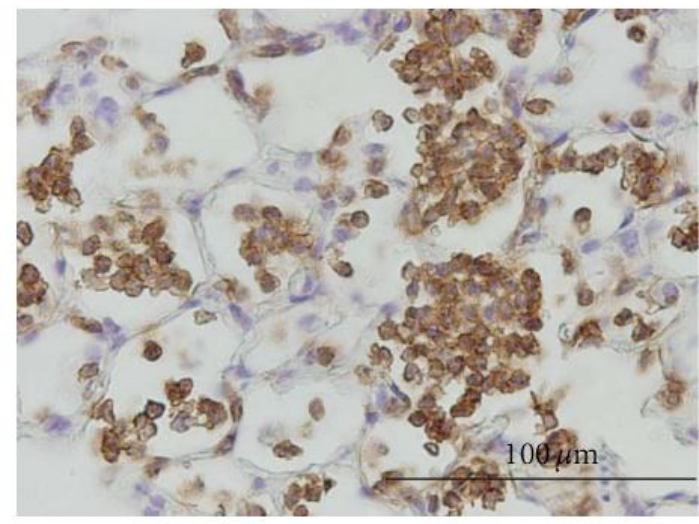

C

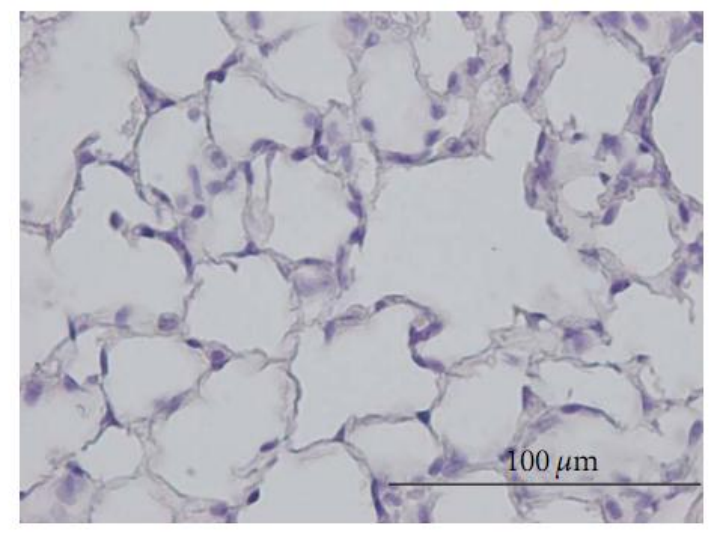

B

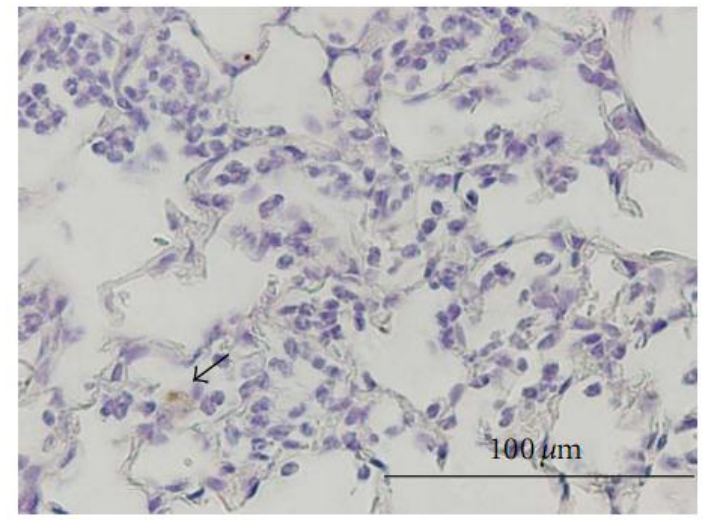

D

Figure 1-5. Immunohistochemical staining of neutrophils (stained with a rat antimouse $\mathrm{IgG}$ ) and macrophages (stained with anti MAC-2 IgG) in lung tissue excised from a control and a $K$. pneumoniae infected mouse (42 hr post challenge). Immunostained cells appear dark brown. Control lungs revealed no neutrophils (A) or macrophages (B), only normal alveolar wall structure. Infected lungs had significant neutrophil accumulation $(\mathbf{C})$, with very little macrophage infiltration (D, indicated by arrowheads). (Magnification, x400.) 
In comparing whole-lung $\mathrm{K}_{\mathrm{i}}$ values to voxel-wise $\mathrm{K}_{\mathrm{i}}$ values for $K$. pneumoniaechallenged mice shown in Figure 1-4A, there appears to be a discrepancy. This can be attributed to the fact that Patlak analysis was done on select axial slices that best highlighted lung inflammation, thus skewing the apparent average lung $\mathrm{K}_{\mathrm{i}}$ value compared to whole-lung $\mathrm{K}_{\mathrm{i}}$ values estimated from the three-compartment model. This is demonstrated in Figure 1-4B which shows the result of Patlak analysis performed on a single voxel (indicated by arrow) and all voxels contained in the lung field. The $\mathrm{K}_{\mathrm{i}}$ value computed for the single voxel, which is located in an area corresponding to intense FDG uptake, is significantly elevated compared to the Patlak-derived whole-lung $\mathrm{K}_{\mathrm{i}}$, leading to the skewing effect described above. The average $\mathrm{K}_{\mathrm{i}}$ value for the control lung (Figure 1-3) derived from multiple slices averaged over six mice is comparable to the average that can be inferred from a single slice in the voxel-wise Patlak plot (Figure 1-4A).

The biggest concern regarding the use of FDG for monitoring activated neutrophils is the specificity of the PET imaging signal. There have been some previous studies conducted to help clarify this question. One particular study chemically depleted neutrophils in mice and measured lung FDG uptake following a subsequent inflammatory challenge [21]. Results from this study showed that when neutrophils are depleted the magnitude of FDG sequestration is reduced in the endotoxin-challenged lung. This finding is consistent with the hypothesis that neutrophils, likely due to their increased metabolic demand during respiratory burst, are the dominant cell type responsible for FDG uptake. Our immunohistochemical analysis, which revealed that neutrophils are the predominant cell infiltrating the lung interstitium and alveoli at the time point studied, supports this hypothesis. 


\subsection{CONCLUSION}

Compartmental modeling is considered the gold standard technique for quantifying FDG uptake by activated neutrophils in the lung. The primary goal of establishing a completely noninvasive method of deriving the blood input function from mice to allow three-compartment modeling of FDG tissue kinetics was accomplished in this study. Using this IDIF, we measured a significantly higher net FDG uptake rate in the lungs following bacterial challenge compared to control lungs. Furthermore, the mean lung $\mathrm{K}_{\mathrm{i}}$ values computed here were not significantly different from previously reported values for mice of similar age and background strain computed from input functions obtained by arterial sampling. To better elucidate the spatial distribution of inflammatory activity, PET data can be transformed into parametric maps of local $\mathrm{K}_{\mathrm{i}}$ using the IDIF with Patlak analysis. FDG-PET is already widely used for cancer detection and assessing response to treatment. In the context of inflammatory lung diseases, this imaging method may be useful for surveillance of neutrophil activation and its noninvasive nature makes it more accessible for use in the preclinical setting. 


\section{CHAPTER 2}

\section{Evaluation of a Novel Neutrophil-Specific}

\section{Peptide for PET Imaging}




\subsection{INTRODUCTION}

Imaging with PET following injection of FDG provides a sensitive index of pulmonary neutrophil metabolic activity in vivo. However, the use of FDG to probe neutrophil metabolic activity in the lungs will always incur uptake by other immune cells, lung parenchymal cells, and normal tissue such as myocardium, potentially leading to false interpretations. To address this problem, we focused on developing an alternative neutrophil imaging approach based on the peptide N-cinnamoyl-F-(D)L-F-(D)L-F (cFLFLF), which was previously reported as an antagonist to the neutrophil formyl peptide receptor (FPR) with a high binding affinity $\left(\mathrm{K}_{\mathrm{d}}=2 \mathrm{nM}\right)$ [43]. However, due to its high hydrophobicity it demonstrated relatively poor target-to-background ratios compared to peptide agonists in imaging focal sites of infection in rabbits [44]. To overcome this problem we modified the peptide by conjugating it with a PEG (molecular weight $=3.4 \mathrm{kD}$ ) moiety to enhance its hydrophilicity and improve blood circulation time [36]. To facilitate PET imaging, the PEG was terminated with DOTA to allow chelation of the PET agent ${ }^{64} \mathrm{Cu}$. In this chapter, we conducted in vitro studies to characterize the binding affinity and agonistic properties of this modified peptide towards neutrophils. Once we demonstrated the peptide had the desired in vitro properties, in vivo PET imaging was performed in a mouse model of pulmonary inflammation.

\subsection{EXPERIMENTAL DESIGN AND METHODS}

\subsubsection{Materials}

All chemicals obtained commercially were of analytical grade and used without further purification. ${ }^{64} \mathrm{Cu}-\mathrm{CuCl}_{2}$ was purchased from Isotrace, Inc. The peptide $N$ cinnamoyl-phe-(D)-Leu-phe-(D)-Leu-phe-lys-CONH $\mathrm{CH}_{2} \quad$ (cFLFLFKCONH$\left.{ }_{2}\right) \quad$ was 
synthesized via solid-phase Fmoc method by Biomolecular Research Facility at the University of Virginia, and the structure was confirmed by matrix-assisted laser desorption/ionization time-of-flight (MALDI-TOF) mass spectroscopy. Bifunctional $t$ butoxycarbonyl-protected PEG-succinimidyl ester ( $t$-Boc-PEG-NHS; molecular weight, 3.4 kD) was obtained from Laysan Bio, Inc. 2,2',2",2"'-(1,4,7,10-Tetraazacyclododecane1,4,7,10-tetrayl)tetraacetic acid (DOTA) was obtained from Macrocyclics, Inc. $\mathrm{N}$ hydroxysulfosuccinimide and 1-ethyl-3-[3-(dimethylamino)-propyl] carbodiimide were purchased from Pierce. All other chemical reagents and solvents were obtained from Sigma-Aldrich. Semipreparative reversed-phase (RP) high-performance liquid chromatography (HPLC) was performed on a Varian system equipped with an ultraviolet detector and a Bioscan Flow Count Radio-HPLC detector with an Apollo C18 RP column $(5 \mu \mathrm{m}, 250 \times 10 \mathrm{~mm})$. The mobile phase changed from $40 \%$ Solvent A $(0.1 \%$ trifluoroacetic acid in $80 \%$ water) and $60 \%$ Solvent B (0.1\% trifluoroacetic acid in $80 \%$ aqueous acetonitrile) to $100 \%$ solvent $\mathrm{B}$ at $30 \mathrm{~min}$ at a flow rate $3 \mathrm{~mL} / \mathrm{min}$. MALDITOF mass spectroscopy analysis was performed on samples of peptide products at W.M. Keck biomedical mass spectrometry laboratory at the University of Virginia, and the data were obtained on a Bruker Daltonics system.

Human tumor necrosis factor- $\alpha$ (TNF- $\alpha)$ was procured from PeproTech, and Nformyl-methionine-leucine-phenylalanine (fMLF) was purchased from Sigma. Aliquots of both samples were taken (TNF- $\alpha, 10 \mathrm{U} / \mathrm{mL}$, and fMLP, $10 \mathrm{mM}$ ) and stored at $-20^{\circ} \mathrm{C}$. For every assay the solutions were thawed to ambient temperature and freshly diluted with hepatic arterial (HA) buffer before use. Multiscreen high-throughput screening (HTS) with glass fiber filter (FC) 96-well plates, type C, with $1.2-\mu \mathrm{m}$ glass filters were 
purchased from Millipore. Filtration from 96-well plates was performed under vacuum on Brandel filtration device. The membranes from each well were collected by punching with the Millipore Multiscreen punching instrument. The radioactivity from ${ }^{64} \mathrm{Cu}$-bound ligand was measured with either Minaxi (Packard), Autogamma 5000 series (Packard), or Wallac 1420 Wizard (Perkin-Elmer) $\gamma$-counters. Radioactivity was measured for $1 \mathrm{~min}$ per sample and was not corrected for radioactivity decay.

\subsubsection{Synthesis and Radiolabeling}

cFLFLFK-PEG- $t$-Boc was prepared by incubating a mixture of cFLFLFK $\left(\mathrm{NH}_{2}\right)$ (10 mg, $10.6 \mu \mathrm{mol})$ dissolved in $2 \mathrm{~mL}$ of acetonitrile and $t$-Boc-PEG-NHS(30 mg, 8.8 $\mu \mathrm{mol})$ dissolved in $2 \mathrm{~mL}$ of sodium borate buffer $(0.1 \mathrm{~N}, \mathrm{pH} 8.5)$ overnight at $4^{\circ} \mathrm{C}$. Removal of volatiles under reduced pressure using rotary evaporator afforded a residue which was dissolved in $2 \mathrm{~mL}$ of trifluoroacetic acid and left at room temperature for $2 \mathrm{~h}$ to remove $t$-Boc protecting group. Concentration of the mixture under reduced pressure followed by reconstitution in $50 \%$ actontrile:water $(2.0 \mathrm{~mL})$ yielded stock solution. This solution was subjected to multiple injections $(\sim 5-6)$ on semipreparative RP-HPLC to collect fractions containing pure cFLFLFK-PEG-NH $\mathrm{NH}_{2}$ (retention time, $18.4 \mathrm{~min}$.). The fractions were concentrated under reduced pressure to yield pure sample, which was further characterized by MALDI-TOF mass spectroscopy. cFLFLFK-PEG-NH $\mathrm{NH}_{2}$

$\mathrm{mg}, 3.8 \mu \mathrm{mol}$ ) was dissolved in $1 \mathrm{~mL}$ of $\mathrm{H}_{2} \mathrm{O}$ and the $\mathrm{pH}$ was adjusted to 8.5 with $0.1 \mathrm{~N}$ $\mathrm{NaOH}$. To this solution was added DOTA-NHS (19 $\mu \mathrm{mol}$ in $20 \mu \mathrm{L}$ of water), prepared according to a previously reported method [45]. The mixture was incubated overnight at $4^{\circ} \mathrm{C}$. The solution was subjected to HPLC purification (retention time $16.8 \mathrm{~min}$.) to yield pure cFLFLF-PEG-DOTA(7.6 mg, 43\%). 
The radiolabeling was accomplished by addition of 7.4-29.6 MBq $(200-800 \mu \mathrm{Ci})$

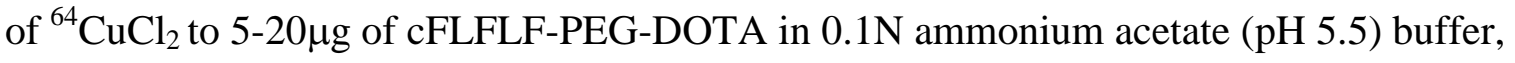
and the mixture was incubated at $40^{\circ} \mathrm{C}$ for $30 \mathrm{~min}$. The mixture was injected as is for RPHPLC purification. The column eluate was monitored by UV absorbance at $215 \mathrm{~nm}$ and with a $\gamma$-detector. The collected product eluted at 17.2 min with a radiochemical yield higher than $95 \%$ and the specific activity of $1.1 \times 10^{6} \mathrm{MBq} / \mathrm{mmol}$ (yield > 90\%). Pure fractions were concentrated under reduced pressure. The radiolabeled peptide was further characterized by comparing its chromatographic properties with non-radioactive copper labeled compound synthesized independently using copper chloride in the same process.

To test for compound stability, we incubated the compound in serum at $37^{\circ} \mathrm{C}$ for 1, 3, and $6 \mathrm{~h}$. After incubation, we monitored the compound with HPLC. To determine the partition coefficient of the PEGylated and non-PEGylated compound, we dissolved about $350 \mathrm{kBq}$ of cFLFLF-PEG 76 -DOTA $-{ }^{64} \mathrm{Cu}$ (or cFLFLF-DOTA $-{ }^{64} \mathrm{Cu}$ ) in $500 \mu \mathrm{L}$ of water and mixed the solution with $500 \mu \mathrm{L}$ of octanol in an Eppendorf microcentrifuge tube. The tube was sonicated for $10 \mathrm{~min}$ and then was centrifuged at $4,000 \mathrm{rpm}$ for $5 \mathrm{~min}$ (Fisher Scientific Marathon Micro-A). Radioactivity was measured in $100 \mu \mathrm{L}$ aliquots of both octanol and water layers in triplicate.

\subsubsection{In Vitro Assays}

Receptor Binding. Human neutrophils were prepared from normal heparinized $(10 \mathrm{U} / \mathrm{mL})$ venous blood by a 1-step Ficoll-Hypaque separation procedure [46, 47], yielding approximately $98 \%$ neutrophils; greater than $95 \%$ viable as determined with trypan blue containing less than $50 \mathrm{pg} \cdot \mathrm{mL}^{-1}$ of endotoxin. After separation, neutrophils 
were washed with Hanks' balanced salt solution with heparin $(10 \mathrm{U} / \mathrm{mL}) 3$ times. After the third wash, neutrophils were resuspended in Hanks' balanced salt solution supplemented with $0.1 \%$ human albumin (Bayer Healthcare). Neutrophil experiments were completed in HA Buffer.

Freshly isolated human neutrophils $\left(4 \times 10^{6}\right.$ cells $\left./ \mathrm{mL}\right)$ were treated with $10 \mathrm{U} / \mathrm{mL}$ of TNF- $\alpha 20$ min before binding studies and transferred to a 96-well plate (Multiscreen HTS FC [Millipore] 1.2- $\mu \mathrm{m}$ glass filter type C, $50.0 \mu \mathrm{L}, \sim 2 \times 10^{5}$ cells per well). Saturation assays were carried out using eight different concentrations of cFLFLF-PEG${ }^{64} \mathrm{Cu}$ (specific activity: $32.9 \mathrm{MBq}[0.89 \mu \mathrm{Ci}] / \mathrm{mmol}$ ) ranging from 0.001 to $100 \mathrm{nM}$. Neutrophils were incubated with the radioligand at $25^{\circ} \mathrm{C}$ for 90 minutes to obtain total binding. After incubation, 96-well plates were filtered rapidly under vacuum using a Brandel filtration device (Brandel Inc.), washed three times with cold Tris-Mg buffer ($5^{\circ} \mathrm{C}, 10 \mathrm{mM}, 150 \mu \mathrm{L}$ each time per well) to remove the unbound radioligand, and dried under a vacuum. The membranes from each well were collected by Millipore multiscreen punching instrument (Billerica, MA). The bound radioactivity remaining on the membranes was measured in a $\gamma$-counter. Specific binding was calculated as the difference between total binding and nonspecific binding. Non-specific binding was assessed using the highest concentration of radiolabeled ligand used in the binding experiment following pre-incubation with cold compound (100 $\mu \mathrm{M}$ of cFLFLF-PEG-Cu). Binding parameters ( $\mathrm{K}_{\mathrm{d}}$ and $\mathrm{B}_{\max }$ values) were calculated using PRISM 4.0 (GraphPad).

Superoxide Production Assay. We used a luminol-enhanced chemiluminescence assay to assess the biological activity of cFLFLF-PEG-copper and fMLP towards human neutrophils. This is a standard assay for measuring the mobilization of myeloperoxidase 
and the formation of reactive oxygen intermediates important in the ability of neutrophils to kill microbes [48]. Purified neutrophils $\left(2 \times 10^{6} / \mathrm{ml}\right)$ were first primed with TNF- $\alpha(10$ $\mathrm{U} / \mathrm{ml})$ and incubated in a water bath $\left(37^{\circ} \mathrm{C}\right)$ for $15 \mathrm{~min}$. Following priming, aliquots of the neutrophils were transferred to a microtitre plate (white-walled clear bottom 96 well tissue culture plates) containing luminol $(100 \mu \mathrm{M})$ and cFLFLF-PEG-Cu or fMLF ranging from $0.0001-10 \mu \mathrm{M}$. Peak stimulated chemiluminescence was determined with a Victor 1420 Multilabel Counter set for chemiluminescence mode using Wallac Workstation software. Sigmoidal dose-response curves for fMLP and cFLFLF-PEG-Cu stimulation of PMN oxidative activity were fit using PRISM 4.0 (GraphPad). Median effective concentrations (EC50) were derived from concentration-response curves using PRISM software. Based on this data we compared the relative agonist potency (EC50) of cFLFLF-PEG-Cu to fMLF on neutrophil oxidative activity.

\subsubsection{In Vivo Assays}

Lung Inflammation Model. Klebsiella pneumoniae strain 43816, serotype 2 (American Type Culture Collection), was grown in trypticase soy broth (TSB) overnight, then subcultured for $2 \mathrm{~h}$ to log-phase growth. Following extensive rinsing, bacteria were diluted in sterile normal saline for inoculation. C57BL/6 mice (male, 8-10 weeks old; Charles River) were inoculated by oropharyngeal aspiration of $50 \mu \mathrm{L}$ of bacterial suspension (approximately $3 \times 10^{5}$ colony-forming units) under light inhalational anesthesia with methoxyflurane. The size of the inoculum was quantitated by plating serial dilutions on MacConkey agar plates and counting colony-forming units after overnight incubation. Mice showed signs of moderate illness 18-36 h after inoculation, when imaging was performed. 
Organ Distribution and Pharmacokinetics. Distribution of radioactivity in the body was determined in both control $(n=4)$ and $K$. pneumoniae-challenged $(n=6)$ mice $18 \mathrm{~h}$ post injection of cFLFLF-PEG- ${ }^{64} \mathrm{Cu}$. After taking a single blood sample from the tail vein, mice were euthanized by deep halothane anesthesia. The pulmonary circulation was flushed with $3 \mathrm{~mL}$ of sterile normal saline via the right ventricle and the following organs and tissues were removed and washed: heart, lungs, muscle, bone, liver, kidney, spleen, small intestine, and stomach. The dissected tissues were placed in a pre-weighed vial and later assayed in a gamma well counter. The measured radioactivity for each sample was decay corrected back to the time of tracer injection. Biodistribution values are expressed as a percentage of the injected activity (\%ID) and normalized for body and organ/tissue mass [49].

Blood kinetics of cFLFLF-PEG- ${ }^{64} \mathrm{Cu}$ was studied in three control mice. Approximately $50 \mu \mathrm{L}$ of blood from the contralateral tail vein was collected in capillary tubes at 5, 15, 30, 60, 120, and 180 minutes after tracer injection $(0.37-0.74 \mathrm{MBq})$. The capillary tubes were placed in a vial that was weighed beforehand and afterward. Activity in each blood sample was measured in a $\gamma$-counter, normalized for injected dose and animal body weight, and expressed as \% ID/g of blood.

Myeloperoxidase Analysis. To estimate the number of intrapulmonary neutrophils, myeloperoxidase assays were performed. Immediately after being imaged, mice were euthanized by deep halothane anesthesia and their pulmonary circulation flushed with $3 \mathrm{~mL}$ of sterile normal saline via the right ventricle. The lungs were removed and snap frozen at $-80^{\circ} \mathrm{C}$ until later assayed. Lungs were weighed and placed in homogenization buffer (hexadecyltrimethylammonium bromide) and homogenized, 
followed by sonication and centrifugation. Five microliters of supernatant was added to the assay buffer (o-dianisidine hydrochloride in potassium phosphate) in a 96-well plate, and optical density kinetic measurements at a wavelength of $490 \mathrm{~nm}$ were made using a $\mu$ Quant (Bio-Tek Instruments, Inc) spectrophotometer. Myeloperoxidase activity is reported as a change in optical density (OD)/min/mg of lung tissue.

Lung Immunohistochemistry Staining. Immunohistochemical analysis was performed on harvested lung tissue $42 \mathrm{~h}$ post $K$. pneumoniae challenge, matching the time point of imaging post infection $(24 \mathrm{~h}$ plus an additional $18 \mathrm{~h}$ of tracer clearance time). Immunohistochemistry was used to assess the relative amount and distribution of neutrophils compared to macrophages in the lungs of control versus infected mice. Before removal of the lungs, the pulmonary circulation was flushed with saline via the right ventricle to eliminate nonadherent white blood cells. The lungs were then inflated with formalin to distend the alveolar spaces uniformly. The trachea was cannulated, and $10 \%$ phosphate-buffered formalin was infused at a pressure of $25 \mathrm{~cm}$ of $\mathrm{H}_{2} 0$. After fixation, the lung was dissected coronally in the plane of the mainstem bronchus. Adjacent histological sections ( $3 \mu \mathrm{m}$ thick) were specifically stained for either neutrophils with a monoclonal rat anti-mouse neutrophil IgG (MCA771G; Serotec) or for macrophages with anti-MAC-2 IgG (ACL8942P; Accurate). Stained cells were observed under a light microscope (Microphot; Nikon, LRI Instruments AB).

\subsubsection{PET/CT Study}

Imaging Protocol. Twenty-four hours after administration of K. pneumoniae, cFLFLF-PEG $-{ }^{64} \mathrm{Cu}(3.7-5.5 \mathrm{MBq}[(100 \sim 150 \mu \mathrm{Ci}])$ in $200 \mu \mathrm{L}$ of saline was injected via the tail vein. Lung SUVs were measured at several time points post injection and fit to a 
monoexponential curve, allowing for the calculation of ligand clearance in the control and infected lungs. This analysis was necessary in order to estimate the time window post injection for which the signal difference between control and infected lungs is maximized.

Animals were placed in a custom imaging tray which permitted reproducible positioning relative to the scanners and allowed anesthesia $(1 \%-2 \%$ isoflurane in oxygen) to be delivered throughout the imaging. Micro-X-ray computed tomography [50] images were acquired using a scanner developed in-house. After CT acquisition, mice were transported to the Focus 120 PET scanner and scanned for approximately 25 min. CT images were reconstructed with a 3-dimensional filtered back-projection algorithm using the COBRA software (Exxim, Inc.). The reconstructed pixel size was $0.15 \times 0.15 \times 0.15 \mathrm{~mm}$ on a $320 \times 320 \times 384$ image matrix. PET data were reconstructed using OSEM-MAP algorithm with 12 subsets and 2 iterations followed by MAP algorithm (18 iterations) using MicroPET Manager (Siemens). The reconstructed pixel size was $0.28 \times 0.28 \times 0.79 \mathrm{~mm}$ on a $128 \times 128 \times 95$ image matrix. All PET images were corrected for decay but not attenuation.

Image Analysis. PET and CT images were co-registered using ASIPRO (Siemens) and a transformation matrix previously obtained with an imaging phantom. To quantify the accumulation of the tracer in lungs, ROI analysis was performed. CT images were used to visualize lung boundaries and guide the placement of lung ROIs, which were drawn manually. $10 \pm 2$ contiguous transaxial lung ROIs were drawn to cover the entire lung field. Lung ROIs were transferred to the PET images and the mean activity per milliliter of lung tissue was determined. SUVs, defined as product of the mean lung 
ROI activity and the animal body weight divided by the injected dose, were computed.

\subsubsection{Data Analysis}

Group data are expressed as the mean \pm SD. The Student $t$-test was used to determine differences in SUV, \%ID, and myeloperoxidase assay results among mice administered $K$. pneumoniae and normal control mice. A $P$ value less than 0.05 was considered statistically significant. Sigma-Stat, version 3.0 (SPSS, Inc) was used for statistical calculations.

\subsection{RESULTS}

\subsubsection{Peptide Synthesis and Radiolabeling}

The desired cFLFLF-PEG-DOTA peptide containing a PEG linker was synthesized in a straightforward manner using a standard synthesis protocol of activating the carboxylic acid group on $t$-Boc protected-PEG with $\mathrm{N}$ hydroxysuccinamidederivatization. Deprotection of $t$-Boc on PEG linker with trifluoroacetic acid afforded a PEGylated peptide with free $\mathrm{NH}_{2}$, which was further acylated with an N-hydroxylated-carboxy derivative of DOTA. At each step the intermediates were purified by RP HPLC and the compounds were characterized by MALDI-TOF mass analysis. The average molecular weight distribution of cFLFLFKPEG-NH $\mathrm{N}_{2}$ was centered at $4.3 \mathrm{kD}$ and major $\mathrm{m} / \mathrm{z}$ peaks were observed at 4240,4284 , 4372, and 4416. The average calculated mass was 4331. Analysis of cFLFLFK-PEGDOTA by MALDI-TOF revealed an average molecular weight distribution centered at $4.8 \mathrm{kD}$ and major $\mathrm{m} / \mathrm{z}$ peaks were observed at $4644,4688,4776$, and 4820 . The average calculated mass was 4718. Finally, cFLFLFK-PEG-DOTA-copper revealed an average molecular weight distribution of about $4.8 \mathrm{kD}$ and major $\mathrm{m} / \mathrm{z}$ peaks were observed at 
4692, 4736, 4778, and 4794. The average calculated mass was 4782, which is in strong agreement with experimental values.

The final radiolabeled cFLFLF-PEG- ${ }^{64} \mathrm{Cu}$ ligand exhibited a pure single peak by RPHPLC chromotography, and its radio purity was assigned to be more than $95 \%$. The radiolabeled compound was compared with its nonradioactive counterpart for additional confirmation of the structure. HPLC analysis yielded no free ${ }^{64} \mathrm{Cu}$ ions or radioligand fragments when testing the stability of the tracer in serum. The partition coefficients of cFLFLF-PEG${ }^{64} \mathrm{Cu}$ and $\mathrm{cFLFLF}^{-64} \mathrm{Cu}$ were measured to be -1.21 and 1.25, respectively, indicating the effectiveness of the PEG in enhancing the compound's water solubility.

\subsubsection{In Vitro Assays}

A saturation curve and Scatchard analysis of cFLFLF-PEG- $-{ }^{64} \mathrm{Cu} \quad$ binding to purified neutrophils is shown in Figure 2-1. From the Scatchard plot a mean $\mathrm{K}_{\mathrm{d}}$
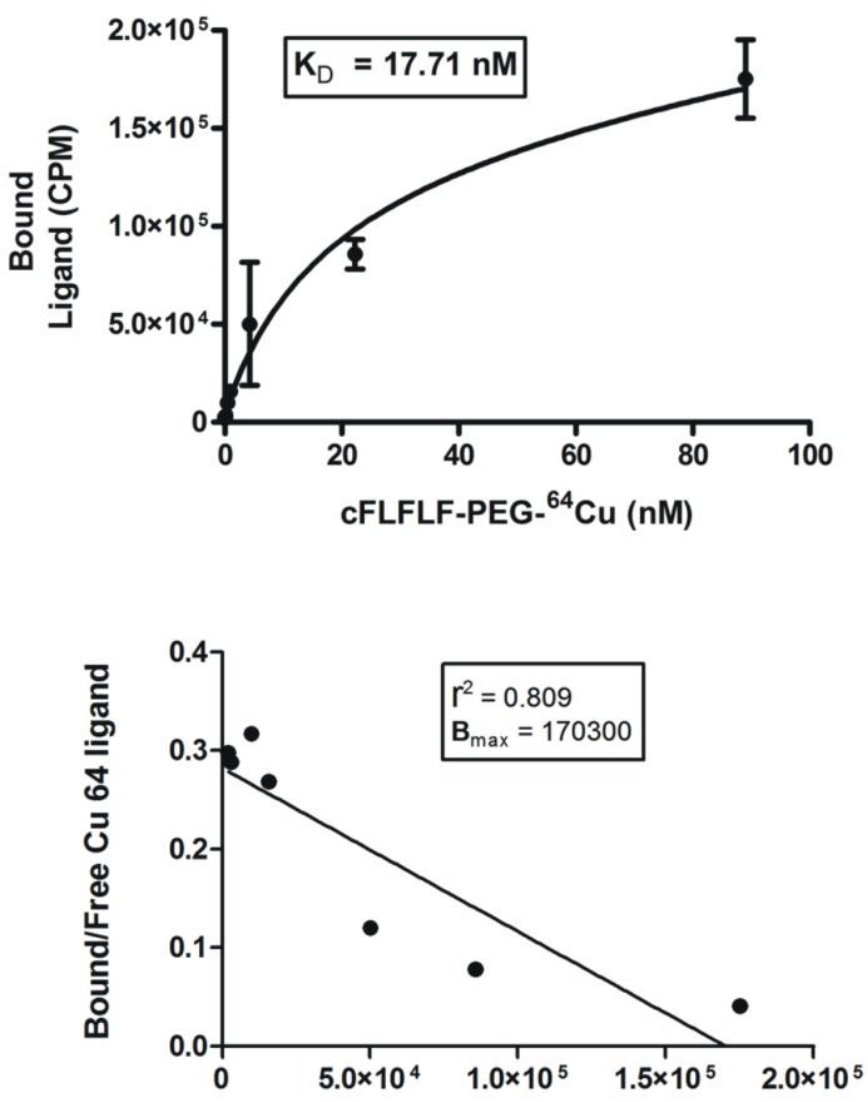

Bound cFLFLF-PEG- ${ }^{64} \mathrm{Cu}$ (CPM)

Figure 2-1. A representative saturation curve of cFLFLF-PEG- ${ }^{64} \mathrm{Cu}$ specifically bound to human neutrophils. Binding affinity was computed to be $17.71 \mathrm{nM}$. Binding data have also been plotted as Scatchard plot. Nonspecific binding was computed in the presence of excess cold compound and is estimated to contribute to $20 \%$ - $30 \%$ of total binding. 


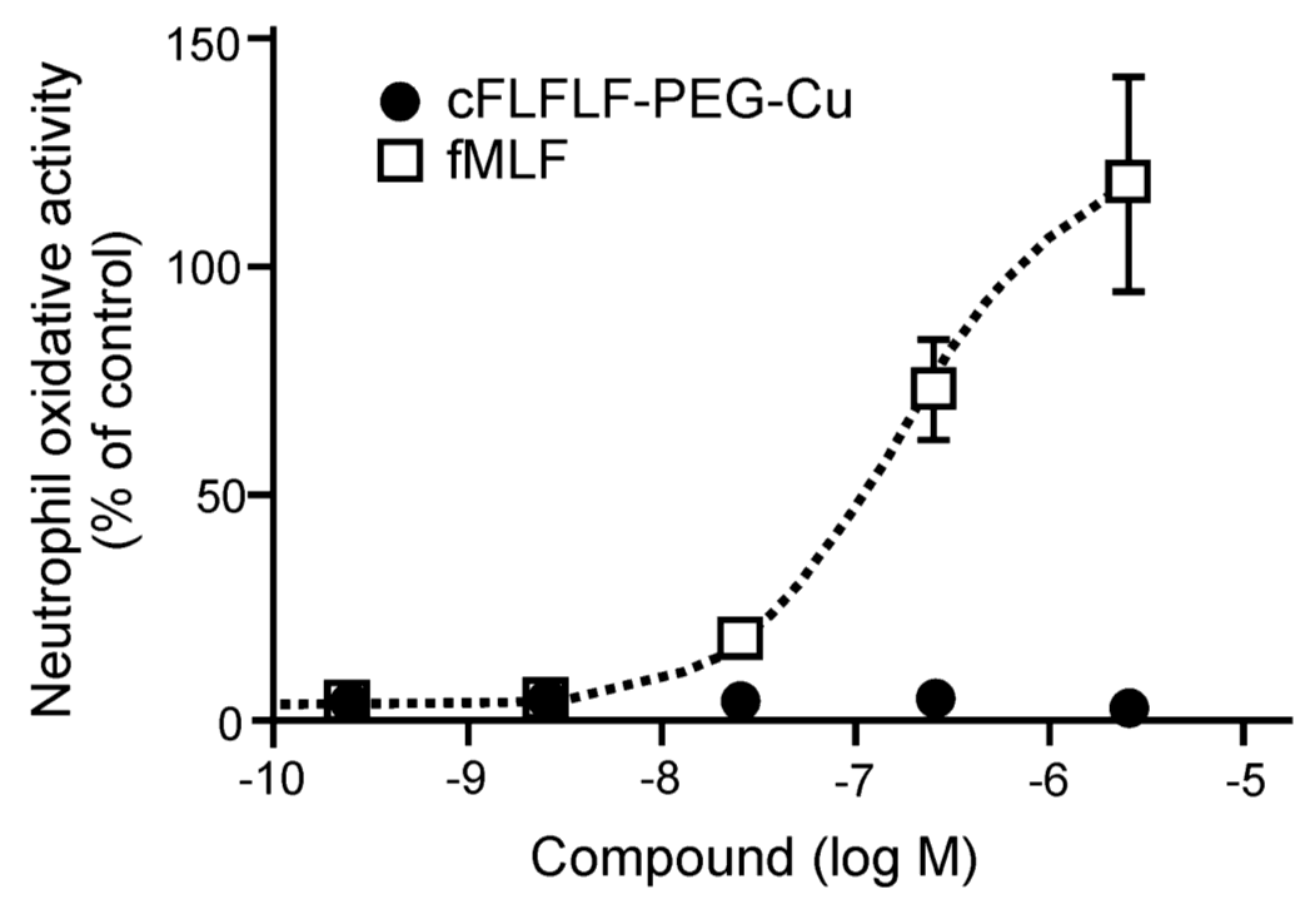

Figure 2-2. Stimulation of the neutrophil oxidative burst by cFLFLF-PEG-Cu and fMLF as detected by luminol-enhanced chemiluminescence. The results are reported as peak chemiluminescence as a percentage of TNF-primed and fMLF-stimulated $\left(10^{-6} \mathrm{M}\right)$ control. cFLFLF-PEG-Cu demonstrated no agonist activity towards neutrophils (EC50 value not computable), while fMLF-stimulated neutrophil superoxide release with an EC50 of $3.7 \times 10^{-7} \mathrm{M}$. Data are reported as mean \pm SEM for at least 2 independent measurements.

value of $17.7 \mathrm{nM}$ was computed. In stimulation assays, cFLFLF-PEG- ${ }^{64} \mathrm{Cu}$ showed minimal agonist activity as assessed by neutrophil superoxide production at all concentrations studied, whereas fMLF exhibited agonist activity with an EC50 of $5.1 \mathrm{x}$ $10^{-7} \mathrm{M}$ (Figure 2-2).

\subsubsection{In Vivo Assays}

Blood Kinetics. The clearance of cFLFLF-PEG- ${ }^{64} \mathrm{Cu}$ in blood followed a monoexponential elimination pattern. The mean biological half-life of the peptide was estimated to be $55 \pm 8 \mathrm{~min}$.

Organ Distribution. Excised tissue concentrations of radio tracer (\%ID) at $18 \mathrm{~h}$ after injection in controls and mice administered $K$. pneumoniae are shown in Figure 2-3. 


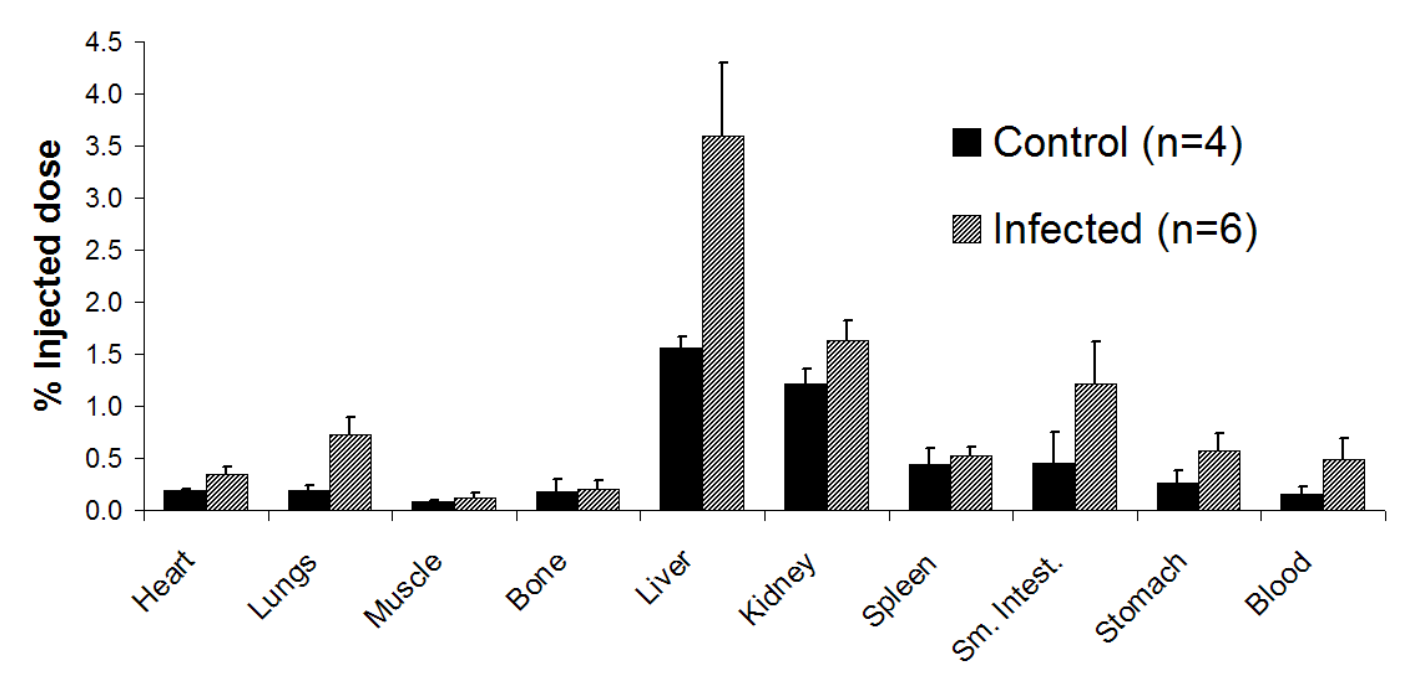

Figure 2-3. Tissue and organ accumulation of cFLFLF-PEG- ${ }^{64} \mathrm{Cu}$ at $18 \mathrm{~h}$ after injection in control and K. pneumoniae-challenged mice, expressed as \%ID/g of tissue. Mean lung radioactivity is 3.8 times greater in infected lungs than in control lungs $\left({ }^{*} P\right.$ $<0.05)$.

For K. pneumoniae-challenged mice, the highest mean concentrations were found in the liver, kidney, and small intestine. The following organs (or tissue) demonstrated statistically significant differences in mean \% ID values between control and infected mice as determined by Student $t$ test: heart, lungs, liver, kidney, small intestine, stomach, and blood. Muscle, bone, and spleen did not exhibit statistically significant differences at the time point studied. The mean ratio of radioactivity in the infected to control lungs was 3.8.

Imaging Results. The imaging properties of cFLFLF-PEG- ${ }^{64} \mathrm{Cu}$ were studied in control $(\mathrm{n}=4)$ and $K$. pneumoniae-challenged mice $(\mathrm{n}=6)$ mice. To establish the optimal time for imaging after ligand injection, the rate of washout in control and infected lungs was computed. Serial PET imaging revealed a half-life of $4.8 \pm 0.7 \mathrm{~h}$ and $10.3 \pm 2.9 \mathrm{~h}$ in control lungs and infected lungs, respectively. From these clearance halflives, the optimal imaging time window post injection was determined to be between 14 and $20 \mathrm{~h}$. 


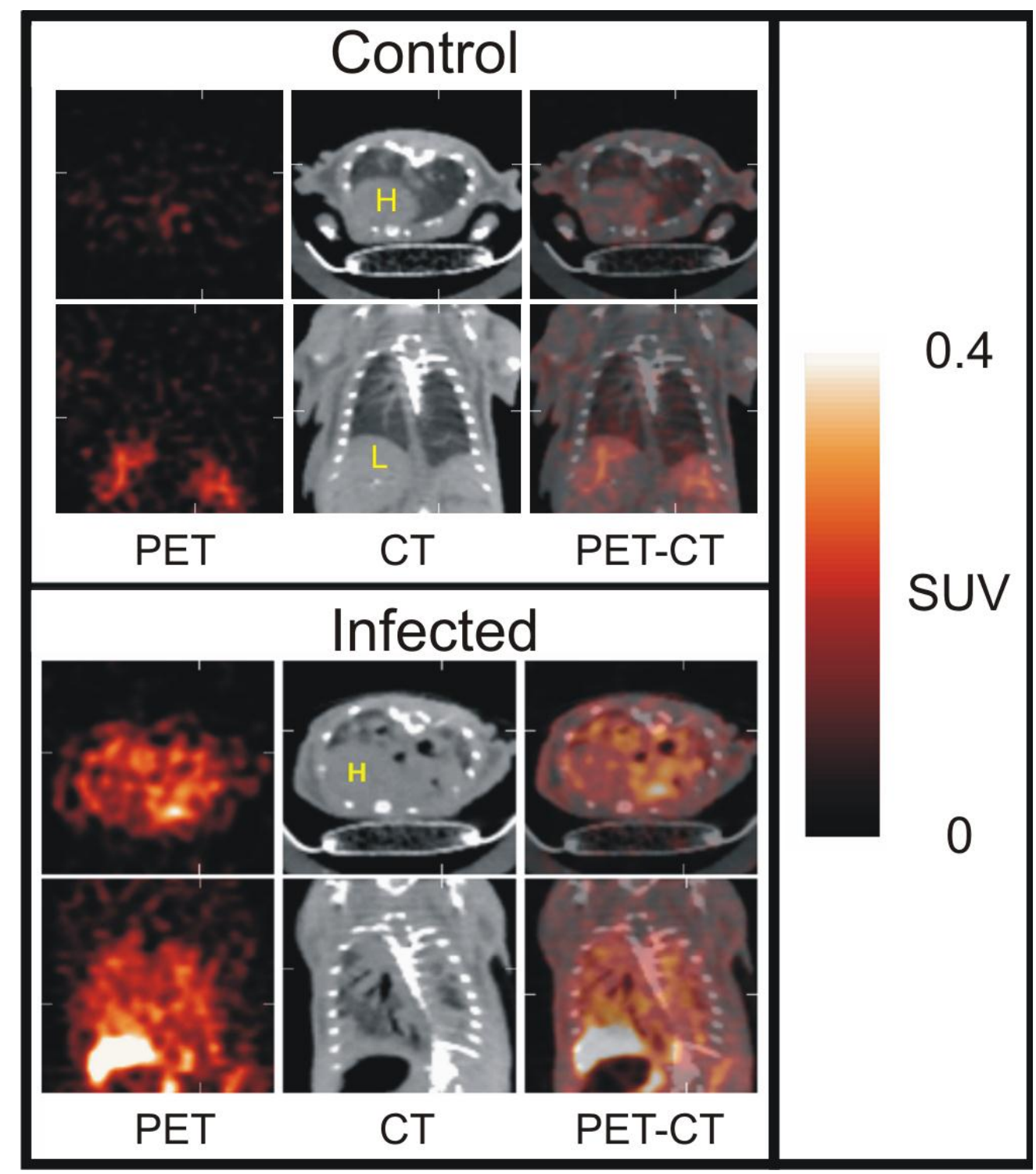

Figure 2-4. Examples of CT and PET images of control and $K$. pneumoniaechallenged mice. PET images were obtained $18 \mathrm{~h}$ after i.v. injection of cFLFLF-PEG ${ }^{64} \mathrm{Cu}$. Both transverse (top row) and coronal (bottom row) images are shown. In mice administered $K$. pneumoniae, there is extensive attenuation in lungs on CT scans, compared with controls. PET scans revealed that $K$. pneumoniae-challenged mice had visually more tracer uptake in lung tissue than did controls, as was quantified by ROI analysis. $\mathrm{H}=$ heart; $\mathrm{L}=$ liver. 
Therefore, the $18 \mathrm{~h}$ time point was chosen out of convenience. Figure 2-4 shows representative CT and PET images of cFLFLF-PEG- ${ }^{64} \mathrm{Cu} 18 \mathrm{~h}$ after injection. These images show significant tracer accumulation in the lungs of the $K$. pneumoniaechallenged mouse, compared with the control mouse. Average lung SUVs for $K$. pneumoniae-challenged and control mice were $0.142 \pm 0.054$ and $0.028 \pm 0.003$, respectively $(* P<0.003)$, as shown in Figure 2-6. High liver uptake was observed in the PET images regardless of whether lung infection was present. The mean lung SUV ratio in infected versus control mice was approximately 5.8 .

Myeloperoxidase Analysis. Myeloperoxidase values measured in excised lung tissue (after imaging) revealed significantly elevated enzyme activity $K$. pneumoniaechallenged mice compared with controls. Mean myeloperoxidase assay values (measured in change in optical density/min/mg of lung tissue) for $K$. pneumoniae-challenged and control mice were $0.78 \pm 0.17$ and $0.12 \pm$ Figure 2-5. Myeloperoxidase activity in lung $0.78 \pm 0.17$ and $0.12 \pm$ 0.05 , respectively $(* P<$ homogenates, expressed as change in optical density per minute per milligram of tissue. Myeloperoxidase activity measured in $K$. pneumoniae-challenged lungs was approximately 6-fold higher than that measured in control 0.005), as shown in 
Figure 2-5. The ratio of mean myeloperoxidase activity in the lungs of mice following $K$. pneumoniae-challenge versus control lungs was 6.3; slightly higher than the corresponding lung SUV ratio measured from PET images of the same mice (Figure 2-6).

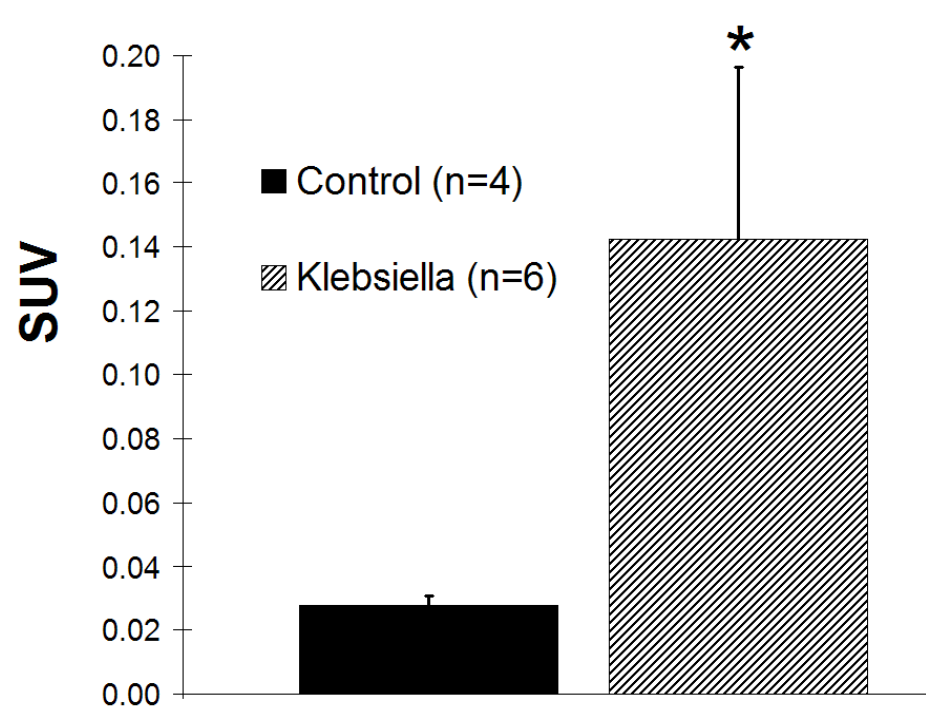

Figure 2-6. $18 \mathrm{~h}$ following injection of cFLFLF-PEG${ }^{64} \mathrm{Cu}$, SUVs measured in K. pneumoniae-challenged lungs were 5.8 times greater in than in control lungs $(* P<$ $0.005)$.

Immunohistochemistry Staining. Immunohistochemical staining of lung tissue from a control mouse revealed very few neutrophils (Figure 2-7A) or macrophages (Figure 2-7B), and normal alveolar wall structure. In contrast, the K. pneumoniae-challenged mouse (euthanized $42 \mathrm{~h}$ after administration) had a significant lung neutrophil burden (Figure 2-7C), with very low numbers of macrophages (Figure 2-7D, indicated by arrowheads).

\subsection{DISCUSSION}

Because the presence of neutrophils and their secretory markers have been strongly linked to the pathogenesis of a number of important pulmonary disease processes, there has been great effort aimed at understanding the role and behavior of these cells in the context of debilitating lung diseases. Initial attempts for tracking leukocytes in vivo with PET imaging utilized in vitro cell labeling with FDG. However, 


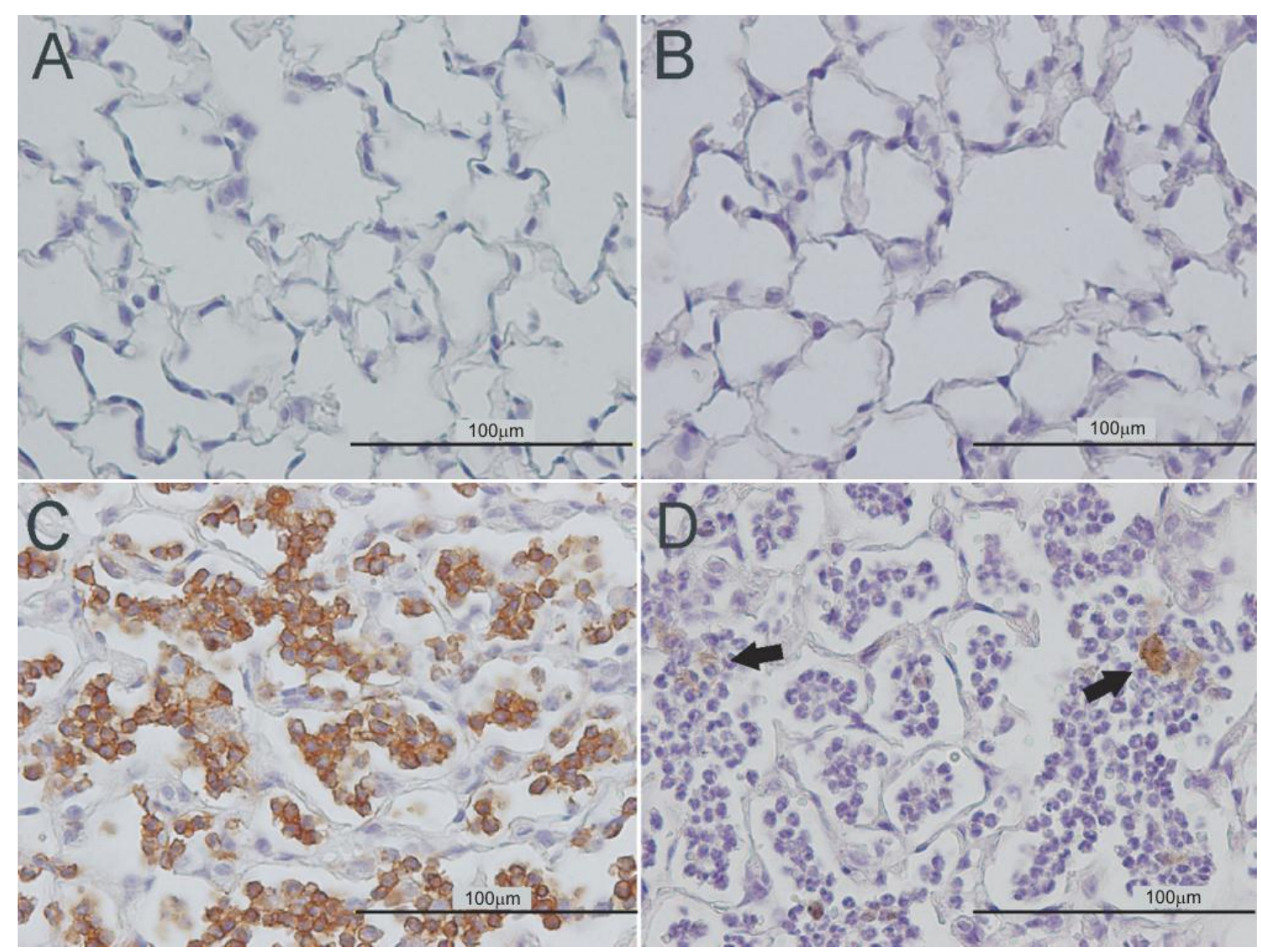

Figure 2-7. Immunohistochemical staining of neutrophils (stained with a rat anti-mouse IgG [MCA771G; Serotec]) and macrophages (stained with anti MAC-2 IgG [ACL8942P; Accurate]) in lung tissue excised from a control and a $K$. pneumoniaechallenged mouse ( $42 \mathrm{hr}$ post administration). Immunostained cells appear dark brown. Control lungs revealed no neutrophils (A) or macrophages (B), only normal alveolar wall structure. Infected lungs had significant neutrophil accumulation $(\mathbf{C})$, with very little macrophage infiltration (D, indicated by arrows). (Magnification, $\mathrm{x} 400$.)

this technique suffers from low and variable labeling efficiency and the short half-life of FDG eliminates the prospect of next-day imaging [51]. Chemotactic peptide receptor agonists have been used successfully in numerous animal studies [52, 53]; however, they are not ideal inflammation imaging agents due to their potential stimulatory effects on leukocytes. Our approach circumvents these problems by relying on in vivo leukocyte labeling with the longer-lived positron emitter, ${ }^{64} \mathrm{Cu}$. The peptide cFLFLF, which has been previously shown to have a high binding affinity towards the neutrophil FPR, does not induce neutrophil activation as do other high-affinity chemotactic peptide analogs 
[43]. Through in vitro studies, we demonstrated that the peptide cFLFLF can be coupled with a PEG moiety for the purpose of enhancing its hydrophilicity without significantly altering its high binding affinity towards the neutrophil FPR. Importantly, the compound showed no biologic activity towards neutrophils as demonstrated by superoxide stimulation assays [36].

SUV measurements derived from in vivo PET images revealed that the localization of the peptide was significantly higher in the lungs of $K$. pneumoniaechallenged mice as compared to control mice $18 \mathrm{~h}$ after injection. We performed myeloperoxidase assays on post-imaged lung tissue to show that this increased signal was primarily due to infiltrating neutrophils responding to the bacteria. Myeloperoxidase analysis confirmed an increased leukocyte infiltration in the bacteria-challenged lungs compared to normal lungs; the magnitude of which correlated well with our SUV measurements. However, because myeloperoxidase is an enzyme that is not exclusively found in neutrophils, we sought additional evidence. Using immunohistochemical analysis, we assessed the relative amounts of neutrophils versus macrophages in both control and infected lungs. From this analysis it was clear that neutrophils were the predominant cell type infiltrating the infected lungs at the $42 \mathrm{~h}$ time point after $K$. pneumoniae administration, with significantly fewer macrophages present.

\subsection{CONCLUSION}

We have demonstrated that the pharmacokinetic properties of the peptide ligand cFLFLF can be improved by PEG conjugation without adversely affecting its binding affinity or stimulatory properties towards neutrophils. Furthermore, we demonstrated using a mouse model of pneumonia that imaging of acute neutrophilic inflammation can 
be achieved with this modified peptide and that the mechanism by which the compound accumulates in the lung appears to be through binding to receptors on infiltrated neutrophils. On the basis of our in vitro binding and stimulation assays and our in vivo PET imaging results, this peptide appears to be a promising new radiopharmaceutical for quantitatively monitoring neutrophilic lung inflammation. 
CHAPTER 3

\section{Liposome PET Imaging of Macrophages in}

\section{Inflammatory Lung Diseases}




\subsection{INTRODUCTION}

Macrophages participate centrally in many pulmonary diseases. In addition to their elevated presence in the lung and alveolar space of patients with COPD $[8,9]$ and $\mathrm{CF}$ $[10,11]$, recent evidence has emerged strongly linking macrophages to many of the physiological processes necessary for the development and progression of lung tumors [54, 55]. Enhancing cell survival, promoting tissue remodeling, angiogenesis, and suppressing the antitumor adaptive immune response are all pro-tumor functions in which macrophages have established roles [54-57]. In animal model studies, the depletion or blockade of tumor-associated macrophages (TAMs) into the tumor microenvironment (TME) has been shown to inhibit tumor growth and reduce tumor vessel density [58-60]. Clinical data have revealed that in many human tumors, including lung, breast, skin, and prostate, a high density of TAMs, specifically of the M2-polarized subtype, correlates with cancer invasion and decreased survival times [14, 55, 61-63]. While it is evident that TAMs represent a unique target for cancer therapies, it appears that the macrophage subtype determines whether they facilitate (M2 subtype) or impede (M1 subtype) tumor growth.

As a result of these observations, methods for monitoring TAMs in vivo are currently being explored to help better understand their role in lung tumor promotion and for assessing macrophage-targeted therapies in the preclinical arena. Molecular imaging using agents targeted to TAMs offers a noninvasive method for monitoring TAM content in the microenvironment and may allow efficient monitoring of response to antiinflammatory therapy. Recently, a dextran-coated Magnetofluorescent nanoparticle decorated with the amino acid glycine (CLIO-gly) was reported to selectively label 
TAMs in vivo [64]. Using fluorescence molecular tomography (FMT) and magnetic resonance imaging (MRI), the authors showed enhanced uptake of the agent within tumors of various murine cancer models. Histological analysis of tumor tissue postimaging confirmed the presence of a large infiltrate of M2 macrophages that co-localized with the in vivo CLIO-gly fluorescent signal. Furthermore, it was shown that the agent could successfully track the depletion of macrophages at the border of implanted tumors following the administration of liposomal clodronate, validating its use for monitoring TAM depletion following anti-TAM therapy [65].

Another recently developed TAM imaging probe known as poly(l-glutamic acid)Gd-chelated p-aminobenzyl-diethylenetriaminepentaacetic acid (PG-Gd-NIR813) was reported that can also be tracked by MRI and FMT [62]. Initially thought to target necrotic areas of tumor tissue, it was later discovered to label TAMs upon reporting a reduced signal following macrophage depletion. Using FMT it was shown that tumorbearing mice pre-treated with a macrophage-depleting agent reduced the tumor uptake of PG-Gd-NIR813 sevenfold compared to tumor-bearing mice that did not receive the depletion drug. Subsequent fluorescence microscopy revealed signal co-localization of PG-Gd-NIR813 fluorescence with newly infiltrated macrophages in subcutaneously injected tumors, providing further evidence of TAM selectivity.

While the mechanism of uptake of CLIO-gly and PG-Gd-NIR813 by TAMs is currently undetermined, there have been other efforts to construct "targeted" liposomes bearing surface carbohydrate residues to interact with specific TAM surface receptors. Surface scavenger receptors, such as the mannose receptor (CD206) over-expressed on TAMs of both human and mouse origins, represent a viable target [66-69]. The mannose 
receptor is important for the clearance of mannose-bearing serum glycoproteins released at sites of inflammation and is known to bind mannose residues on the surface of potentially pathogenic viruses, fungi, and bacteria enabling them to be neutralized by phagocytic engulfment $[70,71]$. Liposomes are naturally recognized by phagocytic cells of the reticuloendothelial system (RES) and can deliver biologically active drugs in their aqueous core or lipid bilayer. Recent work has shown that the inclusion of mannosylated phospholipids in the bilayer membrane enhances liposome uptake by peritoneal macrophages in vivo by mannose receptor-mediated endocytosis $[72,73]$. The goal of this chapter is to develop a PET imaging strategy for targeting TAMs in the lung with mannosylated liposomes. The ability to selectively deliver nanoparticles to TAMs could allow us to detect and quantify their presence, determine and possibly reverse their subtype, and provide a means of delivering select agents to the TME.

\subsection{EXPERIMENTAL DESIGN AND METHODS}

\subsubsection{Animal Model}

Our mouse model is based on one reported by Blackwell, et al. [74]. Female FVB mice (Jackson Laboratory, $\mathrm{n}=6$ ) aged 6-8 weeks received weekly intraperitoneal (i.p.) injections of $1 \mathrm{mg}$ urethane/g body weight dissolved in sterile $0.9 \% \mathrm{NaCl}$. Another group of 3 mice received saline i.p. injections and served as controls. Beginning at 20 weeks after the initial urethane injection, mice were imaged by MRI to verify lung tumor presence and were considered ready for PET imaging when at least one lung tumor reached a size of approximately $1.5 \mathrm{~mm}$ in diameter. The mechanism of lung tumor induction in mice by urethane injection is discussed in Appendix B. 
Prior to conducting studies in vivo, we characterized macrophage infiltration at the tumor border in the urethane-FVB mouse model. Twenty-four weeks after urethane treatment, lungs from a representative mouse were harvested en bloc and inflated with formalin through the trachea. After formalin fixation, the lungs were embedded in paraffin, cut into $2 \mu \mathrm{m}$ slices, and hematoxylin and eosin (H\&E)-stained. Slides were digitized using an Aperio ScanScope scanner and the images were examined for tumors.

To evaluate TAM density relative to macrophage density in normal lung, we immunostained lung sections with rat anti-mouse F4/80 monoclonal antibody (AbDSerotec). We chose F4/80 because it is a well-known, highly restricted macrophage molecule in mice [75]. For this study, lungs from four representative urethane-treated mice were harvested and prepared as described above. To estimate macrophage density, regions of interest (ROIs) were drawn on digitized images in areas corresponding to stroma and normal lung tissue. Within each ROI, the total number of macrophages was manually counted to estimate macrophage density ( $\mathrm{\varnothing}$ per square millimeter). Approximately ten ROIs were drawn in each compartment and the average density was computed.

Finally, we used confocal microscopy to estimate the percentage of $\mathrm{F} 4 / 80^{+}$cells that over-express mannose receptor (CD206), a marker highly expressed on M2 macrophages and minimally expressed on M1 macrophages [76]. Lungs from two representative mice were harvested 24 weeks following the initial urethane injection. After the lungs were inflated with $1.5 \mathrm{~mL}$ of Optimum Cutting Temperature (OCT; Fisher Scientific) through the trachea, the whole lung was snap frozen in liquid nitrogen, embedded into a frozen block, and cryosectioned in a coronal orientation. Frozen lung 
tissue sections were fixed and incubated with rat anti-mouse F4/80 (AbDSerotec) and rabbit anti-mouse CD206 (AbDSerotec). After primary antibody incubation, sections were treated with Alexa Fluor 594-conjugated goat anti-rat IgG and Alexa Fluor 488conjugated goat anti-rabbit IgG. Cell nuclei were visualized by 4',6-diamidino-2phenylindole (DAPI). Confocal images of tumor regions were captured using a fluorescence microscope (Carl Zeiss LSM 700). To estimate the percentage cells within the tumor stroma that co-express F4/80 and CD206, a simple threshold based on staining intensity was applied to several images spanning different tumor regions across two mice.

\subsubsection{Liposome Preparation and Characterization}

Our liposomes were composed of $18.8 \mathrm{mg} / \mathrm{mL}$ of L- $\alpha$-Phosphatidylcholine, 4.2 $\mathrm{mg} / \mathrm{mL}$ of cholesterol, and $0.025 \mathrm{mg} / \mathrm{mL}$ of the lipophilic fluorescent probe 3,3'Dioctadecyloxacarbocyanine Perchlorate $\left(\mathrm{DiO}, \lambda_{\mathrm{ex}}=484 \mathrm{~nm}, \lambda_{\mathrm{em}}=501 \mathrm{~nm}\right.$, Molecular Probes, USA). DiO was included to permit detection by flow cytometry or ex vivo confocal microscopy. The liposomes were made using dehydration-rehydration: the lipids and $\mathrm{DiO}$ were first dissolved in chloroform in a round bottom flask. The solvent in the flask was then evaporated using a Büchi (RE-121) rotary evaporator (Flawil, Switzerland) under vacuum for four hours. Evaporation produces a thin lipid film which could then be hydrated by a $10 \mathrm{mM}$ solution of chelating agent 1,4,7,10-tetraazacyclododecane-1,4,7,10-tetraacetic acid (DOTA) in 10 mM4-(2-Hydroxyethyl)-1Piperazine-Ethanesulfonic Acid (HEPES) buffer containing $150 \mathrm{mM} \mathrm{NaCl}$ with adjusted $\mathrm{pH}$ of 4 for 2 hours at $37^{\circ} \mathrm{C}$ and overnight at $4^{\circ} \mathrm{C}$. The milky solution of liposomes was freeze-thawed 5 times using a dry ice and acetone bath and the solution was extruded consecutively 20 times through $1 \mu \mathrm{m}, 600 \mathrm{~nm}, 400 \mathrm{~nm}$ and $200 \mathrm{~nm}$ polycarbonate 
membrane filters using a Lipex extruder connected to high pressure nitrogen cylinder. The non-encapsulated DOTA was separated from DOTA encapsulated liposomes by dialysis using a Slide-A-Lyzer G2 dialysis cassette with a molecular weight cut-off of 10,000 against five-2 liters of HEPES buffer containing $150 \mathrm{mM} \mathrm{NaCl}$ with adjusted $\mathrm{pH}$ of 7.4.

Mannosylated DOTA encapsulated liposomes (Man ${ }_{3}$-liposomes) were prepared as described above with the inclusion of neoglycolipids synthesized from mannotriose $\left(\mathrm{Man}_{3}\right)$ and dipalmitoylphosphatidylethanolamine (DPPE) by reductive amination. The mannosylated phospholipid was added at a 1:20 $\mathrm{Man}_{3}$-DDPE to phosphatidylcholine (PC) molar ratio and dissolved in chloroform. The mean particle diameter was verified by a laser light scattering particle size analyzer (LS-900, Otsuka Electronics). A schematic diagram of plain and $\mathrm{Man}_{3}$-liposomes is shown in Figure 3-1. These liposomes were manufactured to our specification by Encapsula NanoSciences (Nashville, TN).

The surface availability of mannose residues on $\mathrm{Man}_{3}$-liposomes was verified by measuring changes in UV absorption after the addition of the mannose-binding lectin Concanavalin-A (Con-A) [77]. For this study, $1 \mu \mathrm{L}$ of plain or $\mathrm{Man}_{3}$-liposomes was diluted in $100 \mu \mathrm{L}$ of $10 \mathrm{mMHepes}$ buffer (pH 7.6) containing $150 \mathrm{mM} \mathrm{NaCl}$ and $0.5 \mathrm{mM}$ $\mathrm{CaCl}_{2}$ and loaded into cuvettes. Liposome aggregation was measured by the timedependent increase in turbidity following the addition of $25 \mu \mathrm{g}$ of Con-A as measured by absorbance at $360 \mathrm{~nm}$ using a spectrophotometer.

\subsection{3 $\quad{ }^{64} \mathrm{Cu}$ Labeling}


A technique known as remote loading was used to radiolabel DOTA-containing liposomes with copper-64 $\left({ }^{64} \mathrm{Cu}\right)$, a positron-emitting radionuclide for PET imaging with a radioactive half-life of $12.7 \mathrm{~h}$ [78]. Remote loading utilizes the lipophilic transporter hydroxyquinoline to ferry ${ }^{64} \mathrm{Cu}$ to the liposome interior where it is re-chelated into a more stable complex with the encapsulated DOTA. Following this re-chelation, hydroxyquinoline shuttles back across the bilayer to repeat the process while the hydrophilic ${ }^{64} \mathrm{Cu}$-DOTA complex remains trapped within the liposome interior.

To confirm successful liposome radiolabeling, the ${ }^{64} \mathrm{Cu}$-liposome solution was passed through a size exclusion chromatography (SEC) column to determine if the liposomes eluted in the same fractions as ${ }^{64} \mathrm{Cu} . \quad 10 \mu \mathrm{L}$ of HEPES buffer containing 150 $\mathrm{mM} \mathrm{NaCl}$ and $0.314 \mathrm{mM}$ hydroxyquinoline was added to an Eppendorf tube containing $10 \mu \mathrm{L}$ of radioactive ${ }^{64} \mathrm{Cu}$ solution $(45.8 \mu \mathrm{Ci}$ starting radioactivity). After a brief incubation period, $10 \mu \mathrm{L}$ of DiO-labeled $\mathrm{Man}_{3}$-liposomes was added. Following a $1 \mathrm{~h}$ incubation period at room temperature, the ${ }^{64} \mathrm{Cu}$-liposome solution was passed through a Sephadex G-25 SEC column (GE Healthcare). The goal of using SEC was to determine the amount of ${ }^{64} \mathrm{Cu}$ that was entrapped within liposomes versus the amount of ${ }^{64} \mathrm{Cu}$ that was not liposome associated. Using HEPES buffer as the eluent, $2 \mathrm{~mL}$ of the eluate was collected in individual Eppendorf tubes in $100 \mu \mathrm{L}$ aliquots. The radioactivity of each tube was measured and automatically decay-corrected using a $\gamma$-counter (Wallac 2480 Wizard, Perkin Elmer Life Sciences, Waltham, MA). Immediately following the radioactivity measurement, the collected fractions were transferred to a 96-well plate to measure liposome-associated DiO fluorescence using an IVIS Spectrum Scanner (Caliper Life Sciences, Hamilton, MA). ${ }^{64} \mathrm{Cu}$ radioactivity and $\mathrm{DiO}$ fluorescence were plotted 
together for each fraction collected (approximately 20) to determine if co-elution occurred.

\subsubsection{Culture of Murine BMDM}

To create primary macrophage cultures that mimic physiological and surface marker characteristics of macrophage phenotypes observed in vivo, bone marrow (BM) was flushed from the cavities of femurs and tibiae of FVB mice 8-12 weeks of age. The cell suspension was filtered using a 70-mm filter mesh, centrifuged at $500 \mathrm{x} g$, and resuspended in $1 \mathrm{~mL}$ of MACS buffer (comprising phosphate buffered saline (PBS) supplemented with $1 \%$ bovine serum albumin (BSA) and $2 \quad \mathrm{mM}$ ethylenediaminetetraacetic acid (EDTA)). At this time, four $10 \mathrm{~cm}$ polystyrene Petri plates were prepared each containing $8 \mathrm{~mL}$ of complete media consisting of $\alpha$-Minimum Essential Medium ( $\alpha$-MEM; Gibco) supplemented with 10\% heat-inactivated fetal bovine serum, 10\% CMG14-12 cell conditioned medium (as a source of macrophage-colony stimulating factor (M-CSF)), and 1\% pen/strep(complete $\alpha$-MEM). The cell suspension was then pipetted onto these Petri dishes in equal volumes and cultured at $37^{\circ} \mathrm{C}$ with $5 \%$ $\mathrm{CO}_{2}$ in a humidified chamber for 4 days. This protocol resulted in a population of cells of which $95 \%$ were positive for the mature macrophage marker F4/80, consistent with other reported results [79].

Following the 4 day incubation, adherent cells were treated for $10 \mathrm{~min}$ at $37^{\circ} \mathrm{C}$ with $1 \mathrm{~mL}$ of typsin (Gibco) diluted 1:10 in PBS. Recovered cells were counted using a hemacytometer and re-plated in $60 \mathrm{~mm}$ Petri dishes with $3 \mathrm{~mL}$ complete $\alpha$-MEM at a density of $1.0 \times 10^{6}$ cells per well. Twenty four hours after re-plating, the dishes were divided into two treatment groups. The first group was stimulated with mouse 
recombinant interferon- $\gamma$ (IFN- $\gamma$; PeproTech, Rocky Hill, NJ) and lipopolysaccharide (LPS; Sigma-Aldrich) at a concentration of $20 \mathrm{ng} / \mathrm{mL}$ and $100 \mathrm{ng} / \mathrm{mL}$, respectively. The second group was stimulated with mouse recombinant interleukin-4 (IL-4; PeproTech, Rocky Hill, NJ) plus IL-13 (PeproTech) at a concentration of $20 \mathrm{ng} / \mathrm{mL}$ each [80]. The treatment period for both groups was $24 \mathrm{~h}$. Cells continuously exposed to complete media only were also studied for their mannose receptor expression. In vitro stimulation of murine BM-derived macrophages with IL-4 and IL-13 has been shown to induce M2a or wound healing macrophages similar to those observed in the TME [81-83]. Along with the low production of proinflammatory cytokines, M2-polarized cells are characterized by abundant levels of mannose receptor [84-87]. Therefore, we used Western blot analysis and flow cytometric analysis to assess mannose receptor expression to verify $\mathrm{M} 2$ polarization after cytokine treatment.

\subsubsection{Evaluation of Liposome Uptake by MØ In Vitro}

Uptake of liposomes by macrophages was evaluated by flow cytometry (FACSCalibur, BD Biosciences). MØ(IL-4/13) and MØ(LPS/IFN- $\gamma$ ) were incubated with either $\mathrm{Man}_{3}$ or plain liposomes at a concentration of $960 \mathrm{nmol}$ phospholipid per $\mathrm{mL}$ of PBS at $37^{\circ} \mathrm{C}$ for $90 \mathrm{~min}$. This concentration was established based on a previous titration study discussed in Appendix C. Cells that were incubated with PBS served as negative controls. Following incubation, the cells were washed three times in MACS buffer to remove non-associated liposomes and detached from the Petri dish with NoZyme solution (Sigma). To measure cell surface mannose receptor expression, cells were first incubated with an anti-Fc receptor mAb for 60 minutes on ice to block non-specific binding of antibody to the mouse Fc $\gamma$ domain. Following the block, cells were incubated 
for 20 min with rat anti-mouse CD206 directly conjugated to Alexa Fluor 647 (AbDSerotec). The cells were then fixed with Cytofix (BD Biosciences) and transferred to flow tubes. Two-color flow cytometry was performed with 10,000 events acquired per sample. The fluorescent amplifiers of the FL-1 and FL-4 detector filters were adjusted to ensure that the negative cell population appeared in the first logarithmic decade. Compensation for spectral overlap was not required due to sufficient separation between the fluorescent emission profiles. All experiments were done in triplicate.

FlowJo (TreeStar Inc.) was used to analyze the raw flow data. Macrophages were identified by their light scattering properties. Using negative controls, cellular autofluorescence in both channels was determined by manually defining a gate that maximally included $1 \%$ of autofluorescent cells. Only cells exhibiting a fluorescence intensity above this gate were included in the analysis. CD206 expression was quantified in each treatment group as the geometric mean fluorescence intensity (MFI) of positive cells. Similarly, the geometric MFI of DiO fluorescence associated with M $\varnothing($ IL-4/13) and MØ(LPS/IFN- $\gamma)$ following liposome incubation was measured.

\subsubsection{In Vivo Studies of Liposome Distribution and Uptake by MØ}

To determine the blood half-life of radiolabeled $\mathrm{Man}_{3}$ and plain liposomes, repetitive tail-vein bleeds (about $50 \mu \mathrm{L}$ of blood per sample) were performed at 5 and 30 min, and 1.5, 3, 6, and $18 \mathrm{~h}$ after intravenous (i.v.) injection in 6 normal FVB mice. Administered liposome dose was $1.9 \mu$ mol total phospholipid labeled with1.85 - 2.8 MBq $(50-75 \mu \mathrm{Ci})$ of ${ }^{64} \mathrm{Cu}$ in a total volume of $160 \mu \mathrm{L}$. The radioactivity of each sample was measured using a $\gamma$-counter calibrated for ${ }^{64} \mathrm{Cu}$ energy. In order to estimate how much radioactivity was in the total blood volume at each time point, we assumed that the 
total mass of blood in the mouse is equal to $7.3 \%$ of the body mass [88]. From this we estimated the percentage of the injected dose in total blood mass (\% $\left.\mathrm{ID}_{\mathrm{TBM}}\right)$ at each time point by the following:

$$
\% \mathrm{ID}_{\mathrm{TBM}}=\frac{\left(\frac{\mu C \mathrm{i}_{\text {sample }}}{\mathrm{g}_{\mathrm{sample}}}\right) \times B W \times 0.073}{\mu \mathrm{Ci}_{\mathrm{ID}}} \quad \text { Eq. } 4
$$

where $\mu \mathrm{Ci}_{\text {sample }}, \mathrm{g}_{\text {sample }}, \mu \mathrm{Ci} i_{\mathrm{ID}}$, and $\mathrm{BW}$ represents the radioactivity measured in the blood sample, the mass of the blood sample, the radioactivity of the injected dose (ID), and the body weight of the mouse, respectively.

Twenty four weeks after urethane treatment, lung tumor-bearing mice were i.v. injected with ${ }^{64} \mathrm{Cu}$-labeled $\mathrm{Man}_{3}$-liposomesvia the lateral tail vein. Each mouse received $1.9 \mu$ mol total phospholipid labeled with14.8 - 18.5 MBq $(400-500 \mu \mathrm{Ci})$ of ${ }^{64} \mathrm{Cu}$ for a total volume of $160 \mu \mathrm{L}$. Mice were imaged using a Focus 120 small animal PET scanner (Siemens Molecular Imaging Inc., Knoxville, TN). During the 40 min PET acquisition, anesthesia was maintained using $1.25 \%$ isoflurane in $\mathrm{O}_{2}$ inhaled through a nose cone. Heart rate, respiration, and rectal temperature were monitored using a fiber optic system (Small Animal Imaging Inc., Stony Brook, NY). PET data were reconstructed using OSEM algorithm with 2 iterations and 12 subsets followed by MAP algorithm (18 iterations). The reconstructed image, which was not corrected for attenuation, was composed of 95 axial slices of thickness $0.79 \mathrm{~mm}$ with an in-plane voxel dimension of $0.4 \mathrm{~mm} \times 0.4 \mathrm{~mm}(128 \times 128$ pixels $)$. To determine the optimal time point after i.v. liposome injection for imaging, a preliminary study was conducted in which tumor to normal lung uptake ratios were computed on serial scans acquired over $18 \mathrm{~h}$ in 4 mice. 
Immediately after the PET scan, mice were maintained under anesthesia and imaged by MRI to anatomically localize the PET signal and to identify lung and tumor boundaries on the PET scans. MR imaging was performed on a $4.7 \mathrm{~T}$ MRI system (Varian, Inc., Palo Alto, CA) using a custom-built radiofrequency (RF) coil (Doty Scientific, Columbia, SC). Anesthesia induction and physiological monitoring was the same as previously described for PET imaging. Mice were imaged using a cardiac and respiratory gated multi-slice, spin-echo pulse sequence developed in our lab with the following parameters: field of view $=25.6 \mathrm{~mm}$, effective matrix $=128 \times 128$, slice thickness $=0.6 \mathrm{~mm}, \mathrm{TR}=168 \mathrm{~ms}, \mathrm{TE}=11 \mathrm{~ms}$, number of averages $=4$, number of slices $=10$, and number of interleaves $=4$. The total scan time was 6-8 minutes per interleave, depending on the heart rate and breathing rate. T1-weighted MR images were acquired 15 min after intramuscular administration of Gadolinium-DTPA (Magnevist; Bayer Schering Pharma, Berlin, Germany) at a dose of $50 \mu \mathrm{mol} / \mathrm{kg}$ body weight. Four 10 -slice interleaved stacks were acquired to cover the entire lung field. To facilitate PET-MRI co-registration, a custom-designed multi-modality fusion phantom was previously scanned to determine the rigid-body transformation matrix for fusing subsequent mouse data sets.

Using AMIDE software [89], Man 3 -liposome uptake in tumor and remote lung tissue, liver, and muscle was quantified with PET using co-registered, resolution-matched MR images to guide the size and location of PET ROIs. For lung tumors, ROIs were carefully drawn around the tumor perimeter in each slice for which the tumor was visible. The percent injected activity per $\mu \mathrm{L}(\% \mathrm{ID} / \mu \mathrm{L})$ was computed for each tissue type by dividing the ROI-derived tissue tracer concentration by the radioactivity of the injected 
dose. Due to their small size, ROI-derived radioactivity concentration measurements in lung tumors suffer from partial volume effects (PVE) and as a result they will underestimate the true tumor uptake value. PVE are especially significant when the diameter of the tumor is less than twice the spatial resolution of the PET scanner, which is approximately $1.7 \mathrm{~mm}$ full-width at half-maximum (FWHM) at $0.5 \mathrm{~cm}$ from the center of the field of view for the scanner used in this study [90]. Therefore, we chose to employ a simple correction method based on a phantom experiment. This method consists of multiplying the ROI-derived uptake concentration measured in the tumor by a correction factor called the recovery coefficient (RC). The RC is obtained by determining the fraction of the radioactivity concentration recovered within various-sized rods a previously-imaged PET hot-rod phantom containing a known radioactivity concentration. Based on these measurements, we constructed a look-up table that allows an RC value to be estimated based on the diameter of the object (tumor). Using MRI, we measured the average diameter for each lung tumor and assigned to it an $\mathrm{RC}$ value that was used to correct the ROI-derived radioactivity concentration. ROIs drawn in other tissues such as liver and normal lung were larger than twice the FWHM and therefore were not considered affected by PVE.

\subsubsection{Ex Vivo Tissue Imaging}

Immediately following imaging, the animals were euthanized and the lungs, liver, and spleen were harvested. The lungs were inflated with $1.5 \mathrm{~mL}$ of formalin through the trachea, which was then tied off with a suture. Tissues were washed and rescanned by PET with the same parameters and acquisition time described above. The primary motivation for acquiring ex vivo PET scans was to image the lungs without the presence 
of signal contamination originating from the liver. Fluorescence imaging of the harvested organs was also performed using the IVIS Spectrum to examine the tissue distribution of $\mathrm{DiO}$ and its correlation with ${ }^{64} \mathrm{Cu}$. For DiO fluorescence imaging, we used an excitation filter centered at $500 \mathrm{~nm}$ and an emission filter centered at $540 \mathrm{~nm}$. The lamp level was set to high, binning to medium, field of view to $6.4-12.3 \mathrm{~cm}$ depending on object size, f number to 2 , and exposure time to $0.5-1 \mathrm{~s}$ such that no saturation occurred in the image.

Two representative mice that were administered $\mathrm{Man}_{3}$-liposomes were chosen for further analysis by confocal fluorescence microscopy. For this study, lungs were harvested after in vivo imaging and inflated with $1.5 \mathrm{~mL}$ of Optimal Cutting Temperature (OCT) via the trachea and snap frozen in liquid nitrogen. The lung tissue was then mounted in OCT embedding compound and stored at $-80^{\circ} \mathrm{C}$ until cut into $4 \mu \mathrm{m}$ slices. Tumor-positive frozen tissue sections were fixed and incubated with rat anti-mouse F4/80 followed by Alexa Fluor 594-conjugated goat anti-rat IgG. For visualization of cell nuclei, slides were counterstained with DAPI. Each fluorophore was carefully selected to minimize spectral overlap and potential bleed-through artifacts. Immunofluorescent staining of frozen lung tissue was done by IHC Tech (Boulder, CO). All slides were analyzed using a Zeiss LSM 700 confocal scanning microscope.

\subsubsection{Co-injection Study}

Even though the incorporation of mannotriose into liposome formulations has been shown to enhance macrophage uptake over plain liposomes both in vitro and in vivo [72, 91, 92], we set out to determine the significance of liposome mannosylation for targeting TAMs in vivo in our urethane animal model. The first study, intended to 
address the importance of liposome mannosylation in mediating uptake by TAMs, involved the co-injection of $\mathrm{Man}_{3}$ and plain liposomes (as shown in Figure 3-1) into lung tumor-bearing mice. The second study was identical to the first, with the exception that the plain liposomes were prepared with the inclusion of polyethyleneglycol (PEG)bearing lipids at a molar ratio of 1:20 with respect to PC. The intent of this study is to find out what property is more critical for TAM targeting in vivo by IV injection: the inclusion of PEG which promotes long blood circulation time and retention at the tumor site due to the Enhanced Permeability and Retention (EPR) effect, or the inclusion of

\section{Plain Liposomes}

$\operatorname{Man}_{3}$ Liposomes
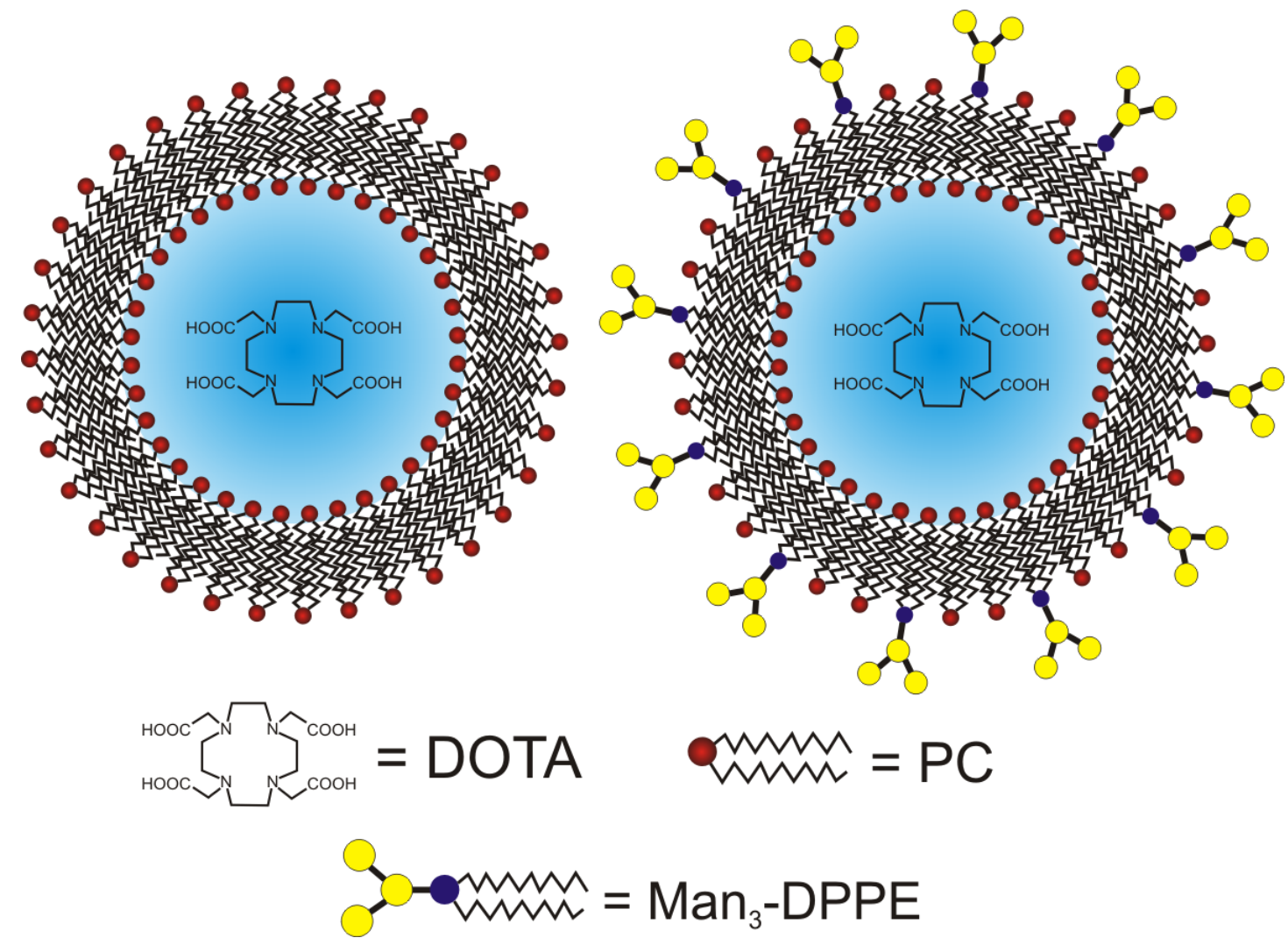

Figure 3-1. A schematic diagram of liposomes. DOTA-containing plain (left) and $\mathrm{Man}_{3}$ (right) liposomes allow for remote loading of the PET imaging agent, ${ }^{64} \mathrm{Cu}$. The mean liposome diameter was $200 \mathrm{~nm}$. 
mannose which may mediate enhanced liposome recognition and endocytosis. In order to independently detect each liposome type based on fluorescence, $\mathrm{Man}_{3}$-liposomes were prepared and labeled with $\mathrm{DiO}$ as described in 3.2.2. However, plain liposomes (with and without PEG) were labeled with a red-shifted lipophilic dye (1,1'-Dioctadecyl3,3,3',3'-Tetramethylindodicarbocyanine (DiD), $\lambda_{\mathrm{ex}}=648 \mathrm{~nm}, \lambda_{\mathrm{em}}=670 \mathrm{~nm}$, Molecular Probes, USA) whose excitation/emission profile was non-overlapping with DiO.

For these in vivo studies, each mouse was tail-vein injected with $1.9 \mu$ mol total phospholipid (approximately $80 \mu \mathrm{L}$ ) of each liposome type plus $80 \mu \mathrm{L}$ of HEPES buffer for a total injected volume of $240 \mu \mathrm{L}$. Six hours after injection, the mice were euthanized and their lungs, liver, and spleen were harvested. Lungs were inflated with $1.5 \mathrm{~mL}$ of OCT via the trachea and carefully arranged on black construction paper along with the liver and spleen. Fluorescent images were acquired using the IVIS Spectrum system. ROIs were manually drawn around the isolated organs using the Living Image 4.0 software package (Xenogen Corp., Alameda, CA). The radiant efficiency for DiO and $\mathrm{DiD}$, defined as the emission light detected (photons $/ \mathrm{sec} / \mathrm{cm}^{2} / \mathrm{str}$ ) normalized by the illumination power density $\left(\mu \mathrm{W} / \mathrm{cm}^{2}\right)$, was measured for each ROI. Tumor-to-remote lung, tumor-to-liver, and tumor-to-spleen ratios were computed in mice co-injected with $\operatorname{Man}_{3}$-liposomes and plain liposomes ( $\mathrm{n}=2$ mice) and $\mathrm{Man}_{3}$-liposomes and PEG liposomes $(\mathrm{n}=2$ mice $)$.

In addition to whole lung ex vivo fluorescence imaging, we also investigated liposome uptake by TAMs at the cellular level. Immediately after imaging, lungs were snap frozen and stored at $-80^{\circ} \mathrm{C}$ until cut into $4 \mu \mathrm{m}$ slices. Select tumor-positive tissue sections were further stained with DAPI and rat anti-mouse F4/80 followed by TRITC- 
conjugated goat anti-rat IgG. Confocal microscopy was used to examine the relative presence of $\operatorname{Man}_{3}$ vs. plain liposomes and $\mathrm{Man}_{3}$ vs. plain PEG liposomes within TAMs. The excitation and emission profiles for each fluorescent dye are shown in Figure 3-15.

\subsubsection{Statistical Analysis}

For comparison of the expression of CD206and liposome association by macrophage treatment groups in vitro, a two-tailed unpaired Student's $t$-test was used for evaluating statistical significance. All data shown are representative of at least 3 independent experiments. The same statistical test was also used to compare tumor to remote lung liposome uptake measured from PET images and macrophage densities in different tissue compartments measured from immunohistochemistry images. $P$ values were considered to be statistically significant when less than 0.05 .

\subsection{RESULTS}

\subsubsection{Validation of Urethane Model}

Twenty-four weeks after urethane treatment, lung tumors were clearly visible on H\&E-stained tissue as shown in Figure 3-2. The majority of tumors were located on the lung pleural surface, an observation consistent with other studies [93]. Quantification of $\mathrm{F} 4 / 80^{+}$cells on immunohistochemistry images revealed that tumor stroma had a sevenfold higher macrophage density compared to remote lung $(673 \pm 196$ vs. $89 \pm 29$ $\mathrm{M} \varnothing / \mathrm{mm}^{2}$, Figure 3-3). It is interesting to note that there was no statistically significant difference between remote lung macrophage density in tumor-bearing mice $(89 \pm 29 \mathrm{M} \varnothing$ $/ \mathrm{mm}^{2}$ ) and macrophage density in normal tissue in a saline-treated mouse (85 $\pm 29 \mathrm{M} \varnothing$ 
$/ \mathrm{mm}^{2}$ ). This result suggests that lung tumors do not influence macrophage density in remote lung compared to that of normal lung in aged-matched, saline-treated mice.

Figure 3-4 shows two contiguous lung sections stained in two different ways. The first section (Figure 3-4A) was H\&E stained which allowed clear identification of tumor and immune cell infiltration in Figure 3-2. A representative photomicrograph of a lung section stained with H\&E 24 weeks

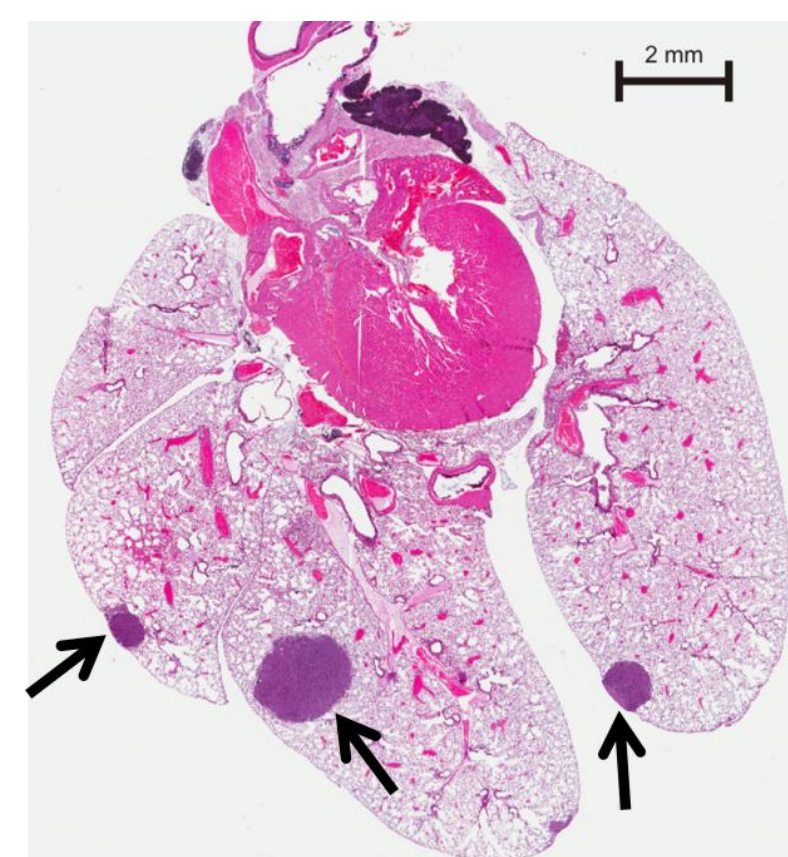
after injection of urethane.

the surrounding stroma. The second section underwent double-immunofluorescence staining to allow simultaneous visualization of F4/80 and CD206 expression in H\&Econfirmed tumor areas. Individual color channels of the confocal image, spatially corresponding to the area in the H\&E-stained image indicated by the black dashed box, are shown in Figure 3-4B. Consistent with immunohistochemical analysis, there was moderate accumulation of $\mathrm{F} 4 / 80^{+}$macrophages at the tumor border and significantly fewer macrophages within the tumor. At the border of the tumor (indicated by the dashed white line), the majority of $\mathrm{F} 4 / 80^{+}$macrophages (red) also stain positive for $\mathrm{CD} 206$ (green), signifying an M2 macrophage phenotype consistent with other reported studies based on a similar urethane model $[67,94]$. 


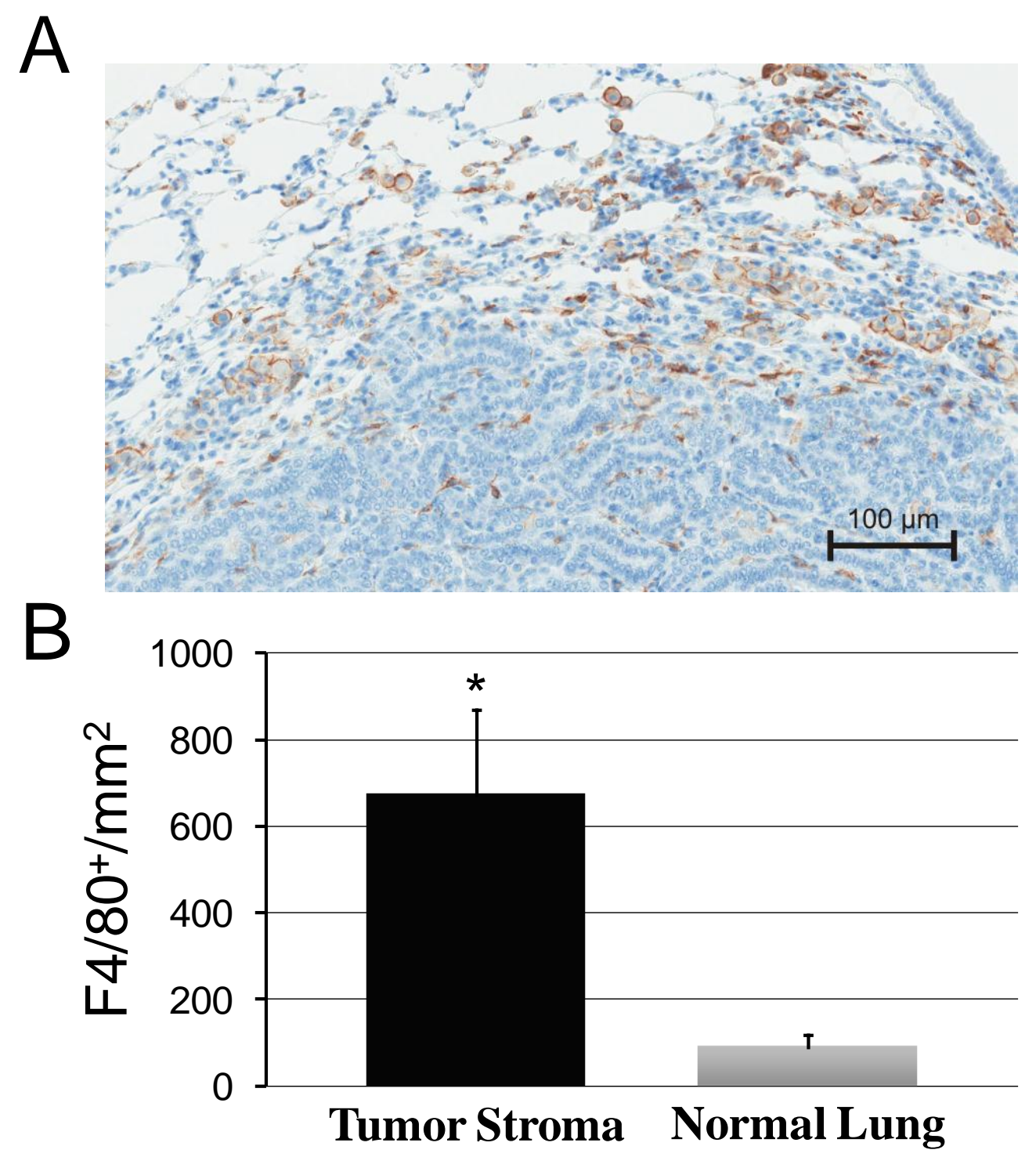

Figure 3-3. Representative IHC staining of F4/80 in lung tissue from a urethanetreated mouse 24 weeks after treatment. As shown in (A), a moderate number of $\mathrm{F} 4 / 80^{+}$macrophages have infiltrated the tumor border, with very few localized within the tumor mass. Quantification of IHC images revealed a seven-fold increase in $\mathrm{F} 4 / 80^{+}$cell density in the peritumoral compartment compared to histologically normal lung $(* P<0.05)(\mathbf{B})$. 


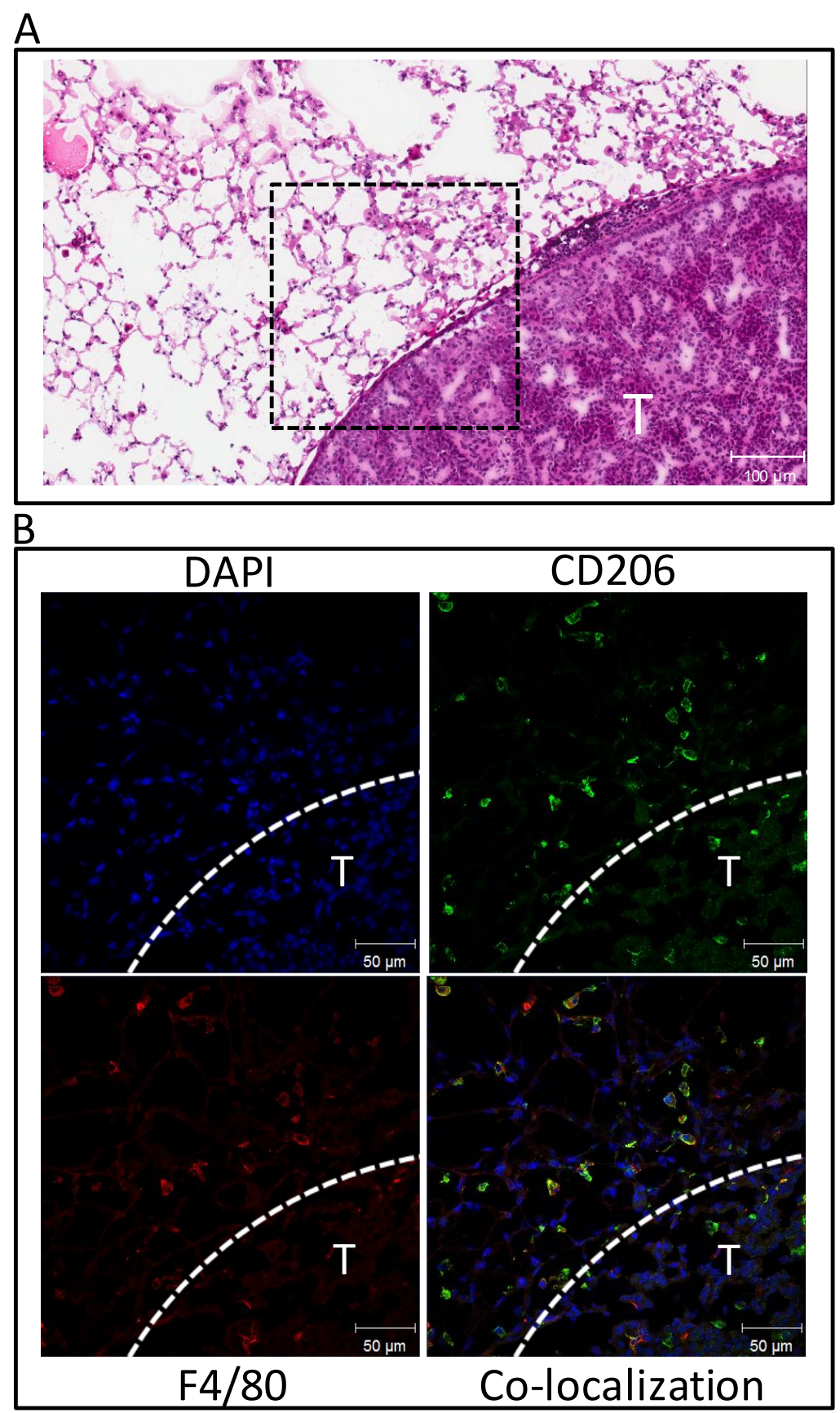

Figure 3-4. Macrophage M2 polarization in lungs of a urethane-treated mouse 25 weeks after treatment. The H\&E image shows a tumor boundary (T) with moderate immune cell infiltration in the stroma (A). Immunofluorescence staining of the tumor stroma outlined in the H\&E image by the black dashed box is shown in (B). At the edge of the tumor (indicated by dashed white line), the majority of macrophages identified by F4/80 (red) also stain for CD206 (green) indicating M2 polarization. 
To estimate the percentage of $\mathrm{F} 4 / 80^{+}$cells that co-express CD206, we used a thresholding technique based on staining intensity. Figure 3-5 shows an example image for which thresholding was applied. On both red (F4/80) and green (CD206) channels, cells were identified with circles and the percent of $\mathrm{F} 4 / 80^{+} / \mathrm{CD} 206^{+}$cells was computed. After pooling together the results from all images, we estimate that $94 \%$ of $\mathrm{F} 4 / 80^{+}$also stain for CD206, indicating that a great majority of macrophages in the tumor stroma are M2-like (Figure 3-5).

\subsubsection{Liposome Characterization}

Successful conjugation of $\operatorname{Man}_{3}$ with DPPE (Man 3 -DPPE) was confirmed by MALDI-TOF mass spectrometry. The $\mathrm{m} / \mathrm{z}$ spectrum demonstrated a strong peak of 1180.6 for the protonated molecule consistent with the theoretical mass value of 1180.7 . As determined by laser light scattering, liposomes exhibited a Gaussian size distribution with the peak of the Gaussian curve aligning with the pore size of the membrane (200 $\mathrm{nm})$. The majority of the extruded population was between 180 and $220 \mathrm{~nm}$.

The plant lectin Con A was used to assess the presence of mannose residues on the liposome surface. Con A is a carbohydrate-binding lectin that binds to accessible mannose molecules resulting in an increase in the mean particle size and solution turbidity. The addition of Con A resulted in 10-fold increase in solution turbidity compared to pre-Con A levels in the formulation containing Man $_{3}$-liposomes (Figure 3-6). Solution turbidity upon addition of Con A indicates that mannose surface ligands were freely accessible to the lectin. In contrast, no absorbance changes were observed with plain liposomes. 
The remote labeling of liposomes by the PET radionuclide ${ }^{64} \mathrm{Cu}$ was verified by SEC. The chromatography profile, shown in Figure 3-7, revealed that the peak of ${ }^{64} \mathrm{Cu}$ elution aligned with the peak of fluorescence (DiO) elution, confirming that ${ }^{64} \mathrm{Cu}$ was entrapped in the liposomes upon exiting the column. The elution of free ${ }^{64} \mathrm{Cu}$ alone occurred much later when passed through the column separately. Nearly $100 \%$ of the

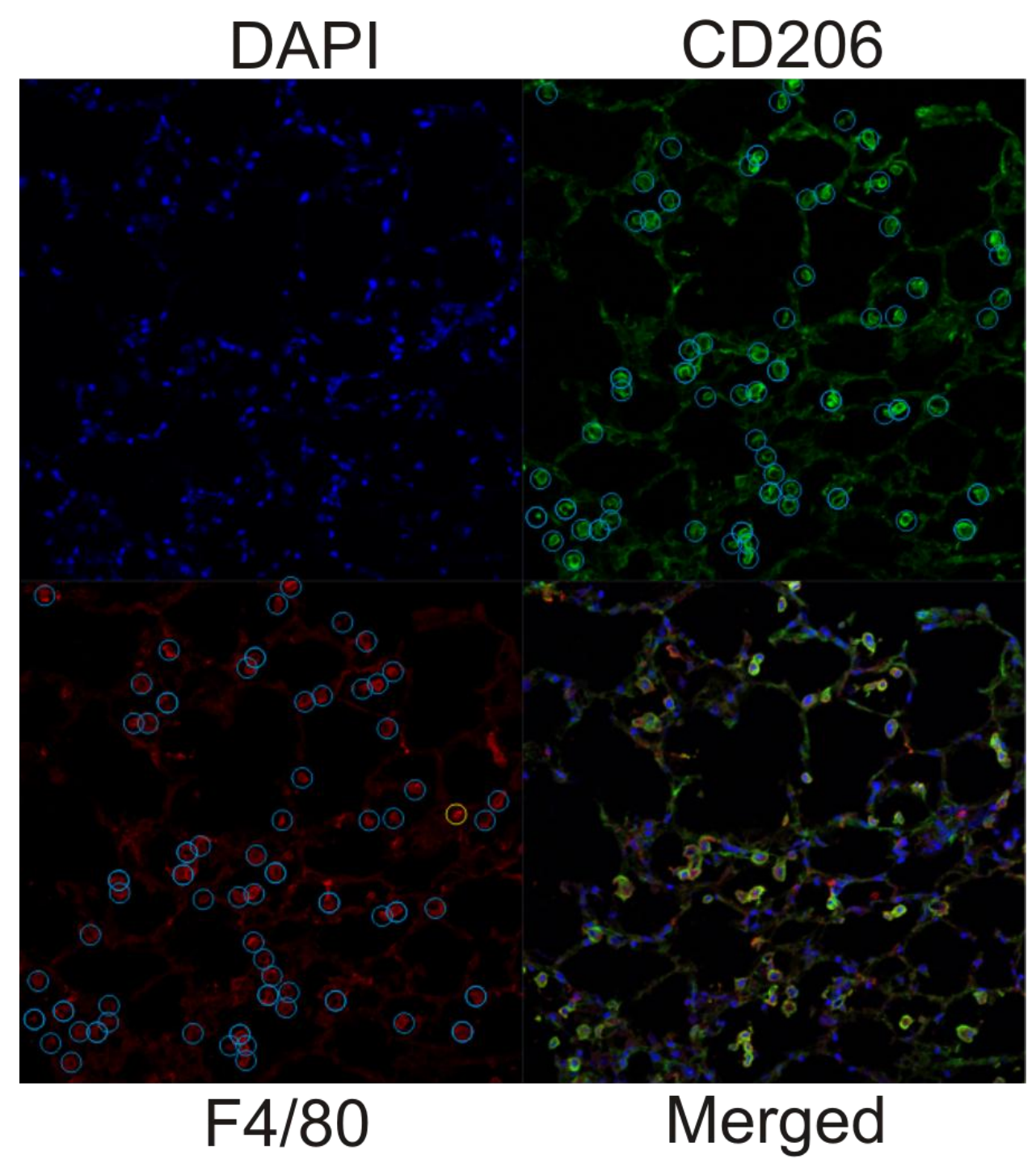

Figure 3-5. Based on a simple threshold, we estimated that $94 \%$ of $\mathrm{F} 4 / 80^{+}$ cells (Red) co-express the mannose receptor (CD206, green), as indicated by blue circles. Yellow circles indicate $\left(\mathrm{F} 4 / 80^{+}, \mathrm{CD} 206^{-}\right)$cells. This finding is consistent with other studies that have shown significant M2polarization of TAMs during a similar phase post urethane treatment. 
starting radioactivity was recovered in the void volume and was associated with the liposome fractions.

\subsubsection{In Vitro Liposome Uptake}

Along with the low production of proinflammatory cytokines, M2 macrophages are characterized by abundant levels of mannose receptor [84-87]. Therefore, prior to examining liposome uptake by MØ(LPS/IFN- $\gamma)$ and MØ(IL-4/13), we first measured mannose receptor expression (CD206) in these treatment groups. As shown in Figure 3-8A and Figure 3-8B, CD206 expression was upregulated on MØ(IL-4/13) compared to $\mathrm{M} \emptyset(\mathrm{LPS} / \mathrm{IFN}-\gamma)$ and MØ(M-CSF) as measured by flow cytometry and Western blotting, respectively. A reduction in CD206 expression by MØ(LPS/IFN- $\gamma$ ) compared to MØ(MCSF) is consistent with other studies that have shown IFN- $\gamma$ stimulation to have a

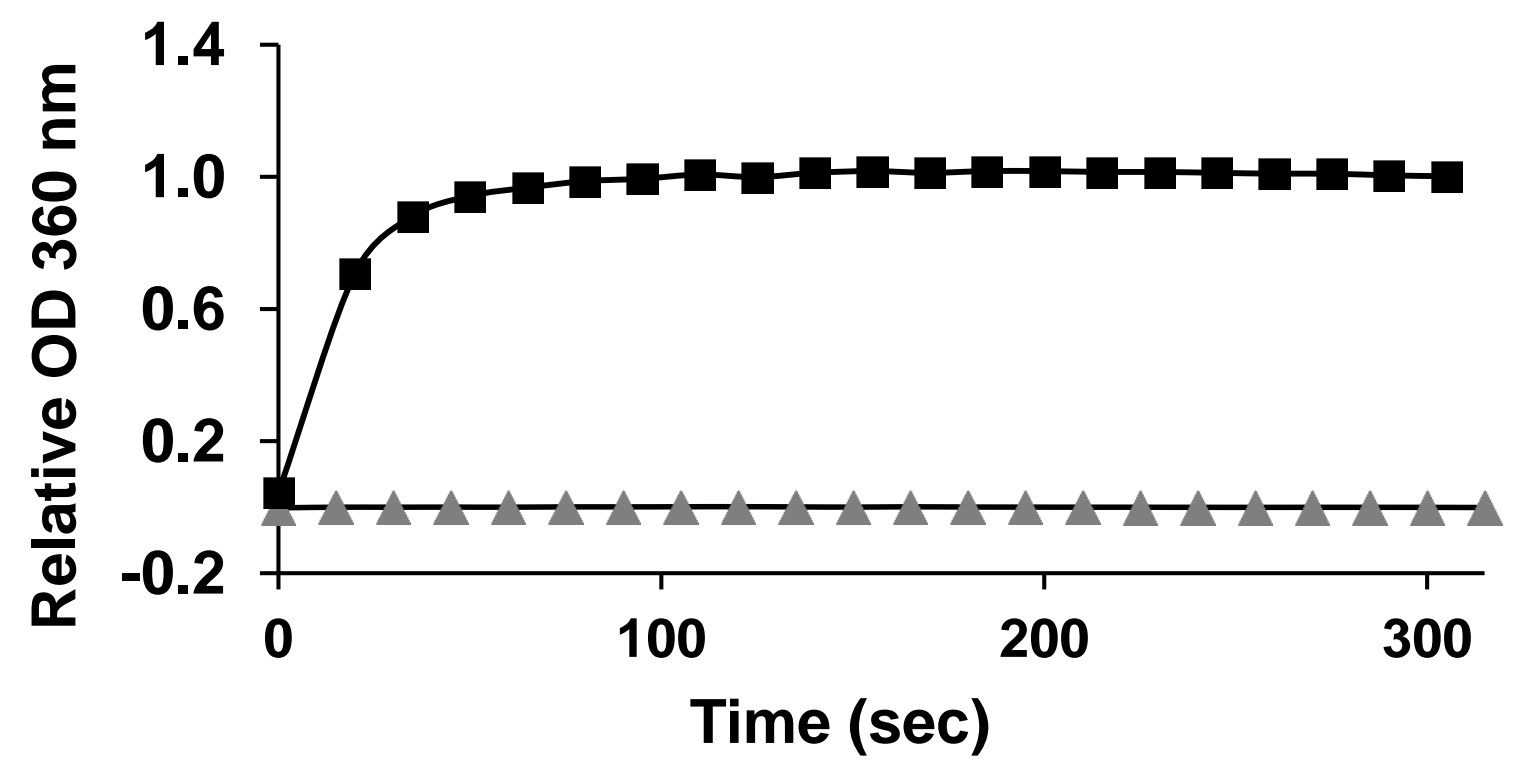

Figure 3-6. Con A-induced agglutination assay of $\mathrm{Man}_{3}$ and plain liposomes. $10 \mu \mathrm{L}$ of Con-A $(25 \mu \mathrm{g})$ was added to $100 \mu \mathrm{L}$ of plain (gray triangles) or $\mathrm{Man}_{3}$ (black squares) liposomes and absorbance at $360 \mathrm{~nm}$ was measured as a function of time. The addition of Con-A to $\mathrm{Man}_{3}$-liposomes resulted in a 10-fold increase in solution turbidity, signifying the presence of mannose residues on these liposomes. In contrast, no change in turbidity was observed for plain liposomes. 
mannose receptor-reducing effect on macrophages in vitro[95].

The uptake of plain and Man 3 -liposomes by MØ(LPS/IFN- $\gamma)$ and M $\varnothing(\mathrm{IL}-4 / 13)$ as assessed by flow cytometry is shown in Figure 3-9. Following identification based on scatter properties, macrophages were analyzed for $\mathrm{DiO}$ fluorescence. Fluorescence histograms of MØ(LPS/IFN- $\gamma)$ and MØ(IL-4/13) following a 90 min incubation with either DiO-labeled plain or $\mathrm{Man}_{3}$ liposomes are shown in Figure 3-9A. These histograms show that $\operatorname{Man}_{3}$-liposomes were more strongly associated with macrophages of both stimulation groups compared to plain liposomes. However, quantification of the flow cytometry data revealed a significant 1.9-fold higher association of $\mathrm{Man}_{3}$-liposomes with MØ(IL-4/13) compared to MØ(LPS/IFN- $\gamma)(P<0.05$, Figure 3-9B). This enhanced

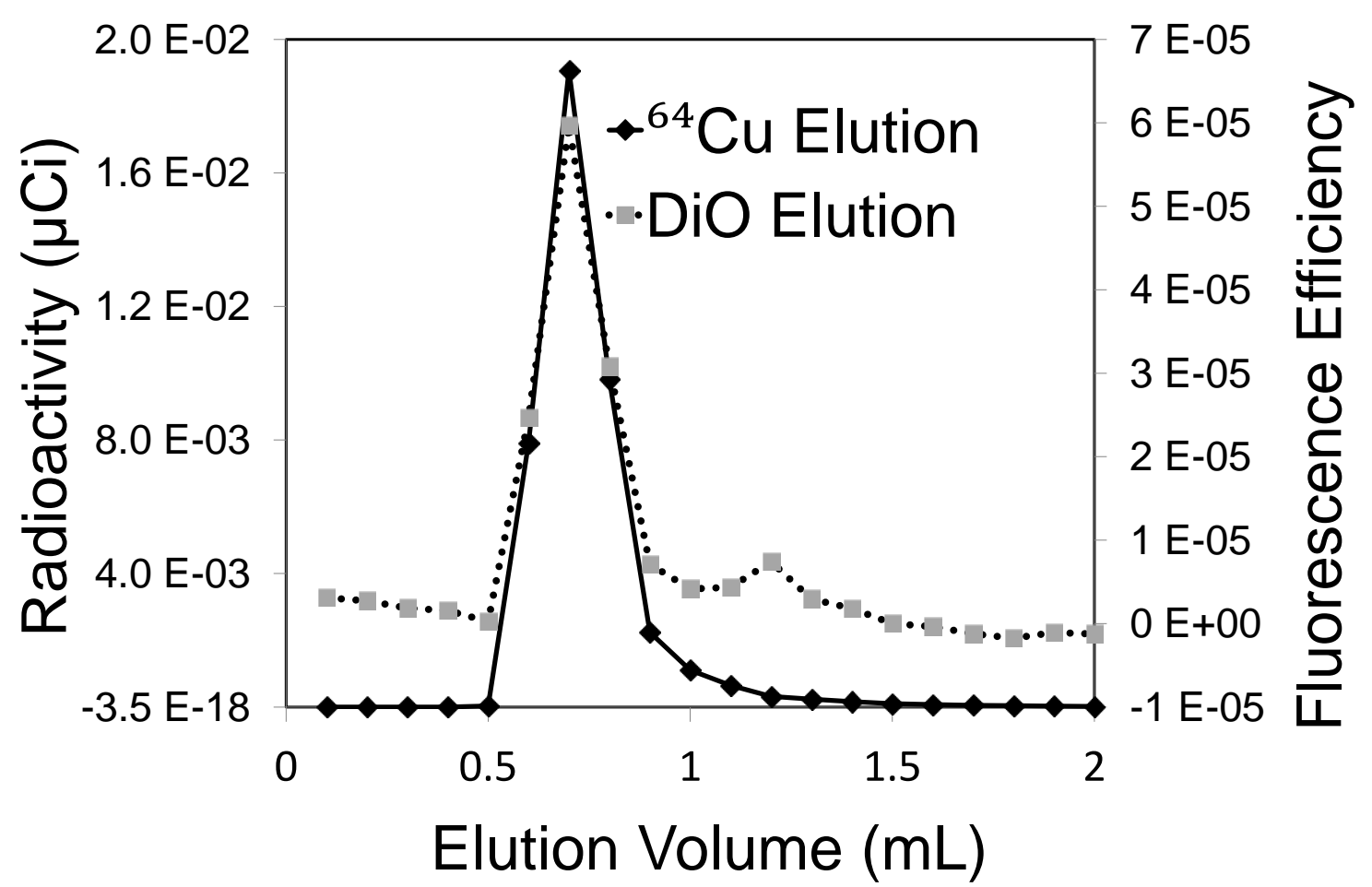

Figure 3-7. An elution profile of ${ }^{64} \mathrm{Cu}$ and $\mathrm{DiO}$ following a $1 \mathrm{~h}$ liposome incubation period with ${ }^{64} \mathrm{Cu}$ and 2-hydroxyquinoline. The profile was constructed by collecting the eluate in $100 \mu \mathrm{L}$ aliquots and measuring the radioactivity and fluorescence. Co-localization of the peaks indicates that ${ }^{64} \mathrm{Cu}$-liposome loading was successful. 


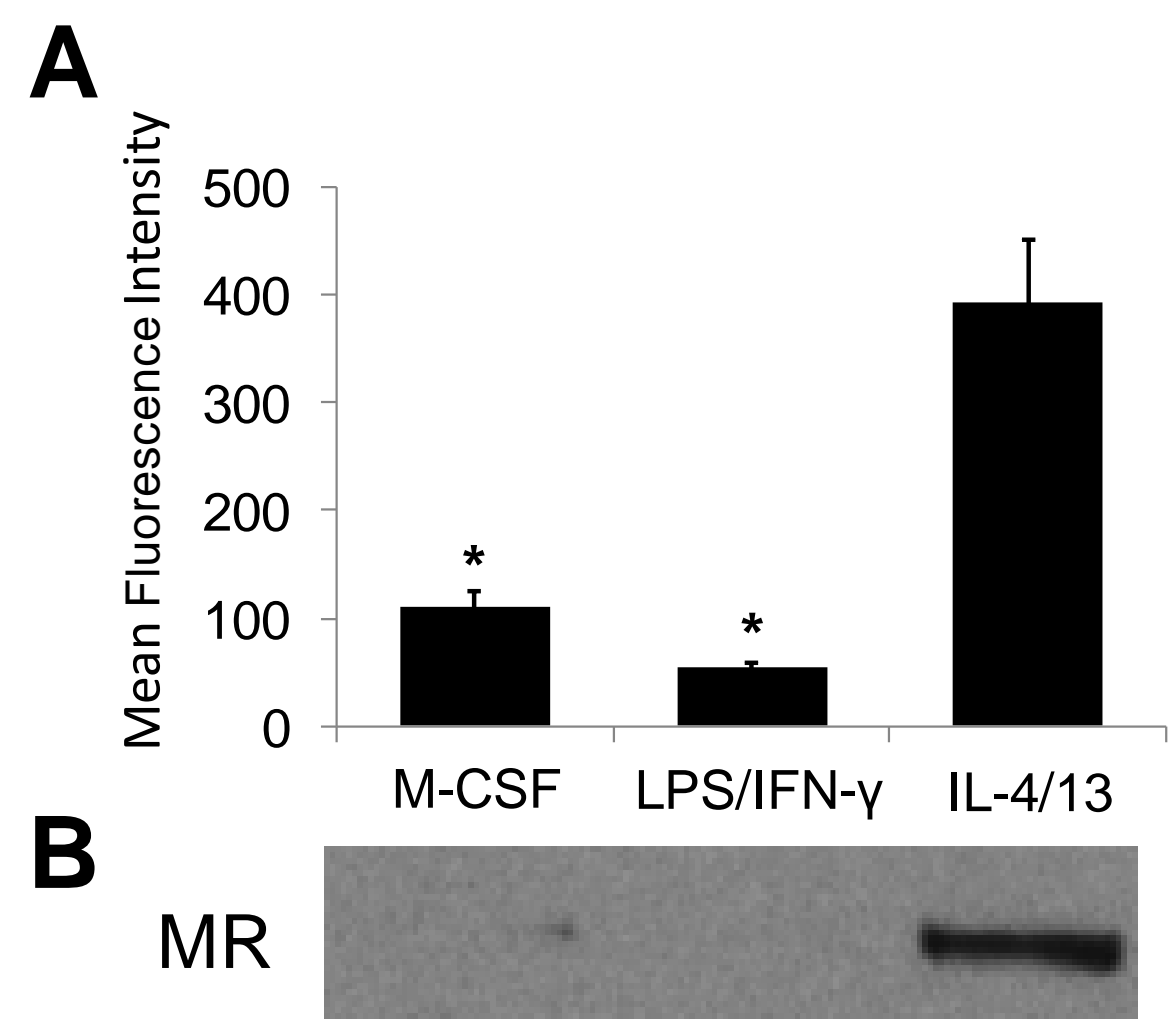

Figure 3-8. Expression of CD206 (mannose receptor) measured by flow cytometry (A) and Western blot analysis (B). Flow data represent average of three trials. Error bars indicate standard deviations. $* P<0.05$ vs. IL-4/13-stimulated cells.

association is likely due to receptor recognition of liposome mannotriose and subsequent mannose receptor-mediated endocytosis.

\subsubsection{In Vivo Studies}

The blood clearance of ${ }^{64} \mathrm{Cu}$ labeled liposomes was studied in normal healthy FVB mice after administration of $10 \mathrm{MBq}$ of radiolabeled liposomes through the lateral tail vein. As shown in Figure 3-10, the blood clearance of both liposomes followed a monoexponential decay with half-lives of 0.33 and $0.23 \mathrm{~h}$ for $\mathrm{Man}_{3}$-liposomes and plain liposomes, respectively. This result is consistent with previously published work which measured a blood half-life of less than 30 min for liposomes of similar size and 


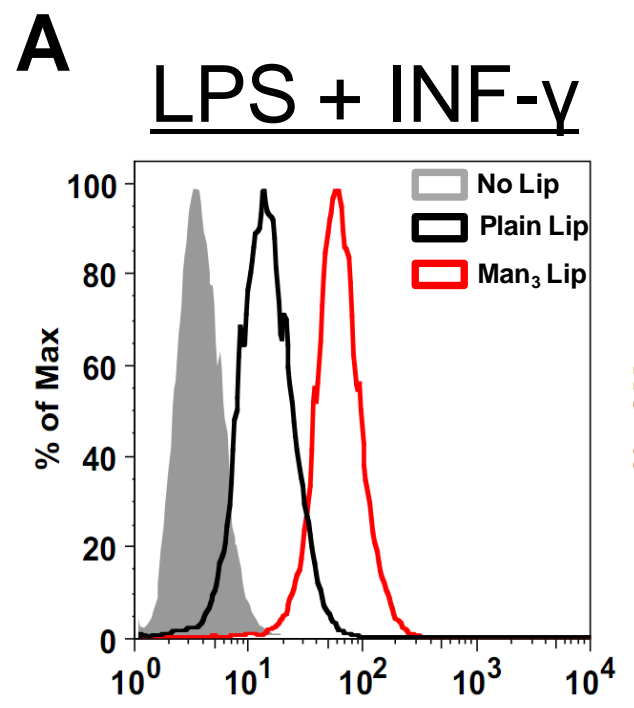

B

DiO Fluorescence

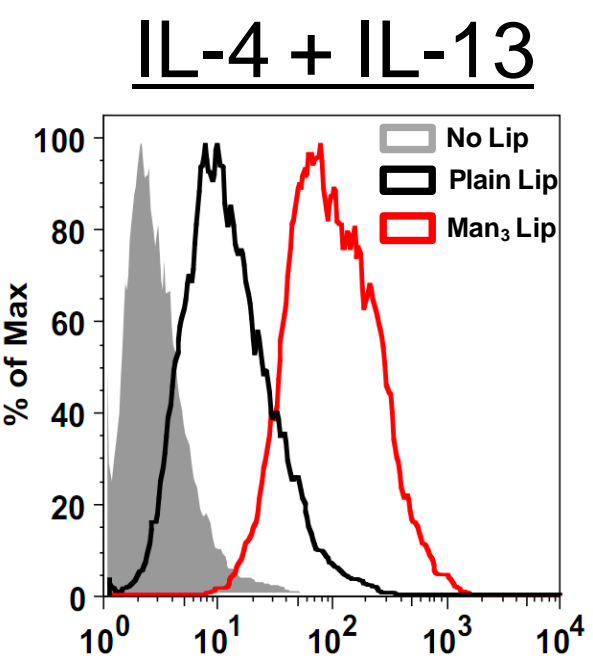

DiO Fluorescence

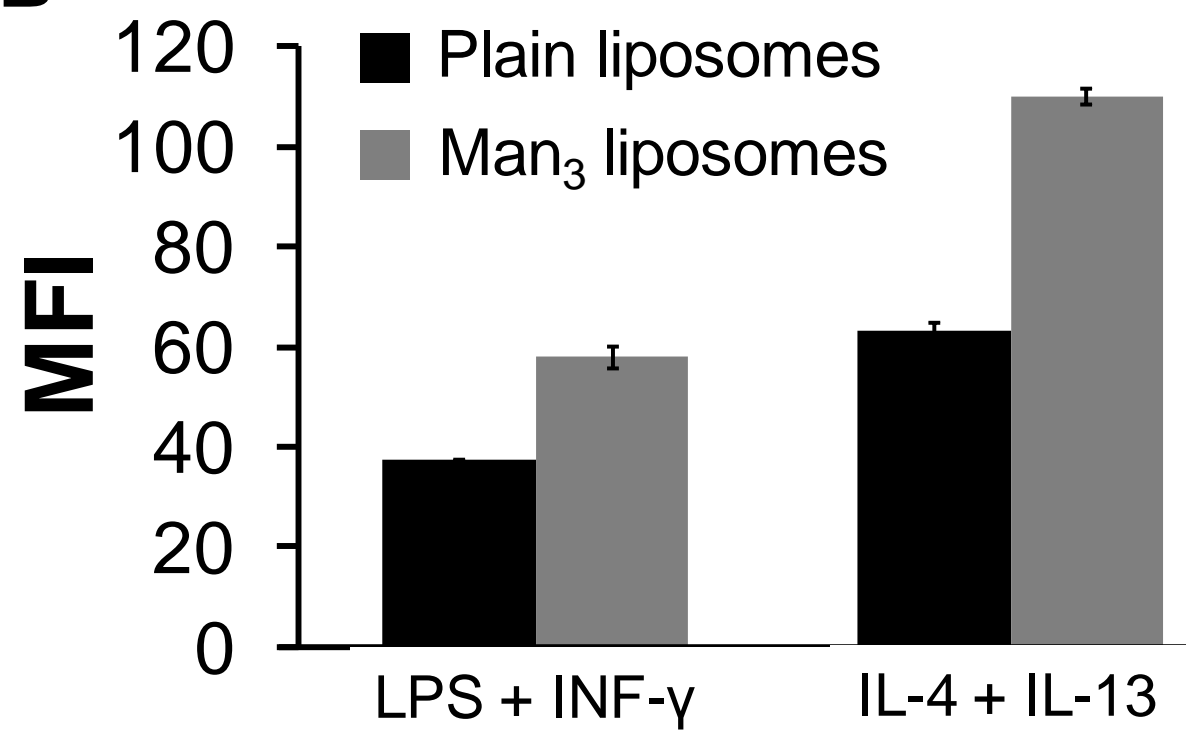

Figure 3-9. Association of DiO-labeled plain and $\mathrm{Man}_{3}$-liposomes with MØ(LPS/IFN- $\gamma$ ) and MØ(IL-4/13) measured by flow cytometry. Cells were incubated with either liposome type for $90 \mathrm{~min}$ after which cell-associated $\mathrm{DiO}$ fluorescence was measured. (A) Representative fluorescence histograms of $\mathrm{M} \varnothing(\mathrm{LPS} / \mathrm{IFN}-\gamma)$ and $\mathrm{M} \varnothing(\mathrm{IL}-4 / 13)$ following incubation with either plain (black histograms) or $\mathrm{Man}_{3}$ liposomes (red histograms). Non-liposome incubated cells (gray histograms) are shown for reference. (B) Geometric MFI values and standard deviation of $\mathrm{DiO}$ fluorescence of all replicates $(n=3)$ are shown. As assessed by the two-tailed Student's $t$-test, $\mathrm{Man}_{3}$-liposomes were associated with MØ(IL-4/13) significantly more than M $\varnothing(\mathrm{LPS} / \mathrm{IFN}-\gamma)$. Likewise, compared to plain liposomes, $\mathrm{Man}_{3}$-liposomes were more strongly associated with M $\varnothing(\mathrm{IL}-4 / 13)(P<0.05$, for both). 
composition (PC and cholesterol) [110].

Urethane-treated mice were imaged by MRI starting at 16 weeks to verify the presence of lung tumors. Examples of coronally acquired spin echo images $30 \mathrm{~min}$ after gadolinium injection of a urethane and saline-treated mouse are shown in Figure 3-11. Several tumors are clearly visible in the lungs of the urethane-treated mouse, which are easily detected relative to normal lung tissue which is nearly void of signal. In contrast, the saline-treated mouse has no detectable lung tumors.

To assess in vivo TAM targeting, ${ }^{64} \mathrm{Cu}$-labled $\mathrm{Man}_{3}$-liposomes were i.v. injected into lung tumor-confirmed mice $(\mathrm{n}=6)$. PET imaging was performed $6 \mathrm{~h}$ following injection. This time point was chosen because it resulted in the highest ${ }^{64} \mathrm{Cu}$ signal ratio between tumor tissue and remote lung while still maintaining sufficiently high

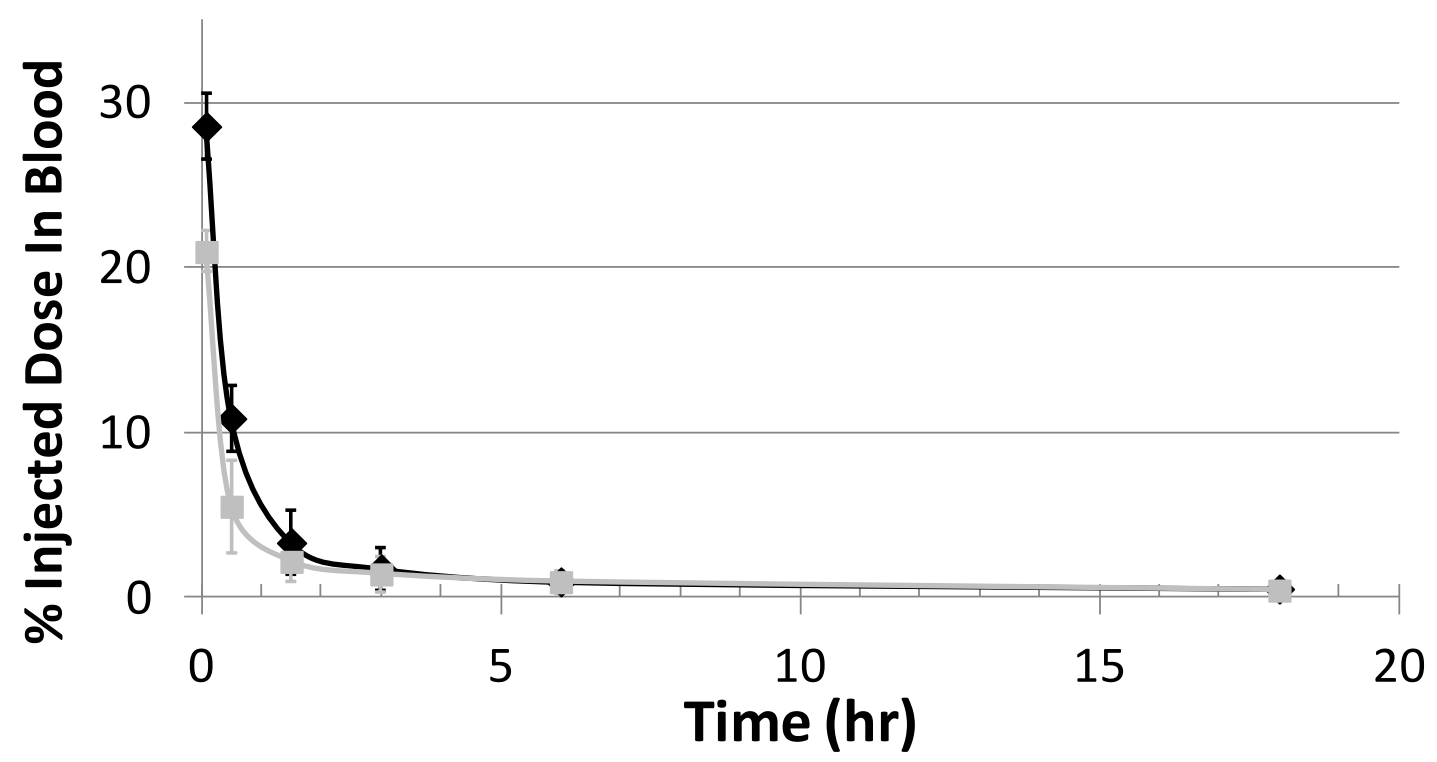

Figure 3-10. Blood clearance curves of Man $_{3}$-Liposomes (black curve) and plain Liposomes (gray curve) measured in normal mice are shown. Liposomes were i.v. injected and the blood was withdrawn at different time intervals. Sample radioactivity concentrations were normalized by the injected dose and multiplied by $7.3 \%$ of the body weight to approximate the \% ID in blood as shown in Eq. 1. Values shown are the mean of three experiments. Error bars represent standard deviation of the mean. 


\section{Saline Treated}

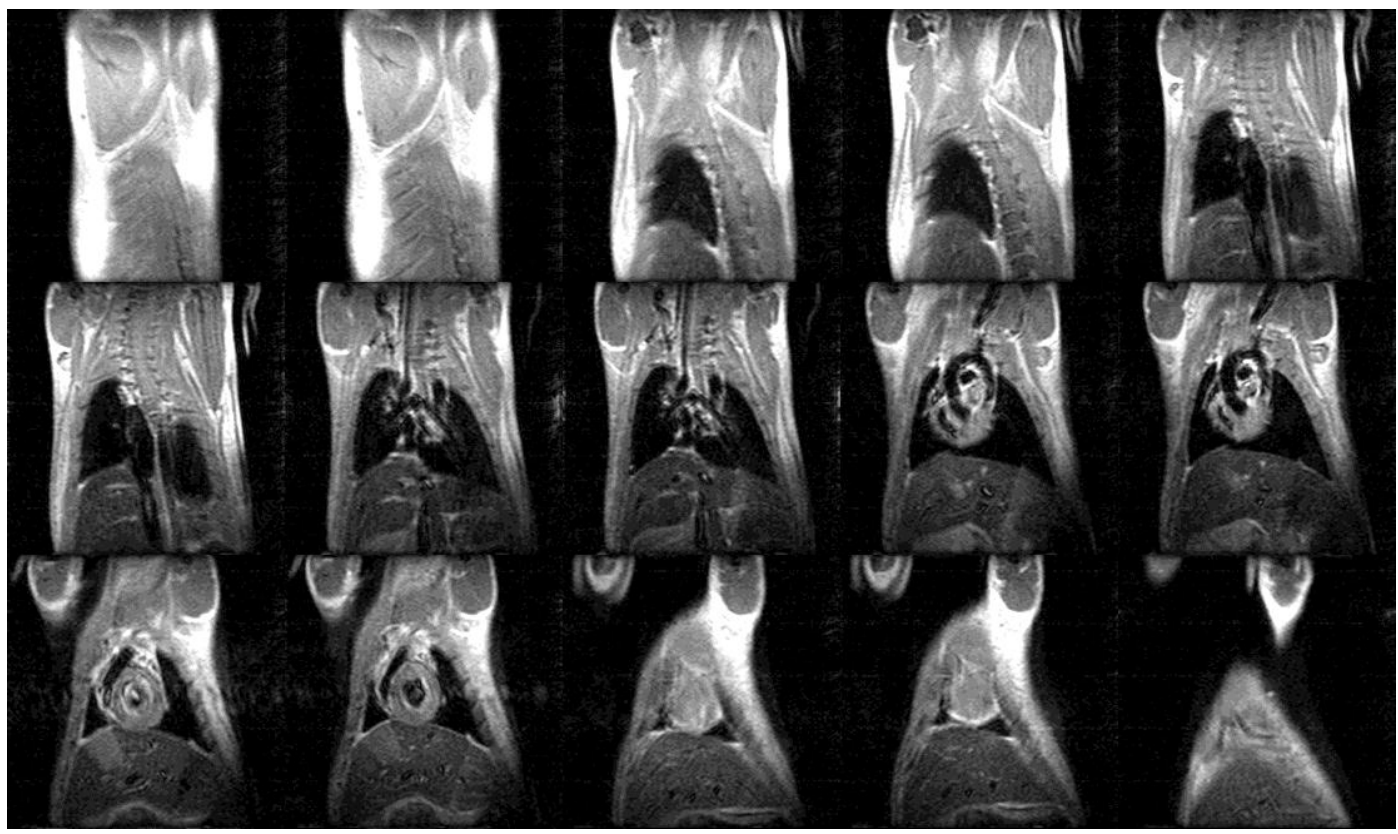

\section{Urethane Treated}

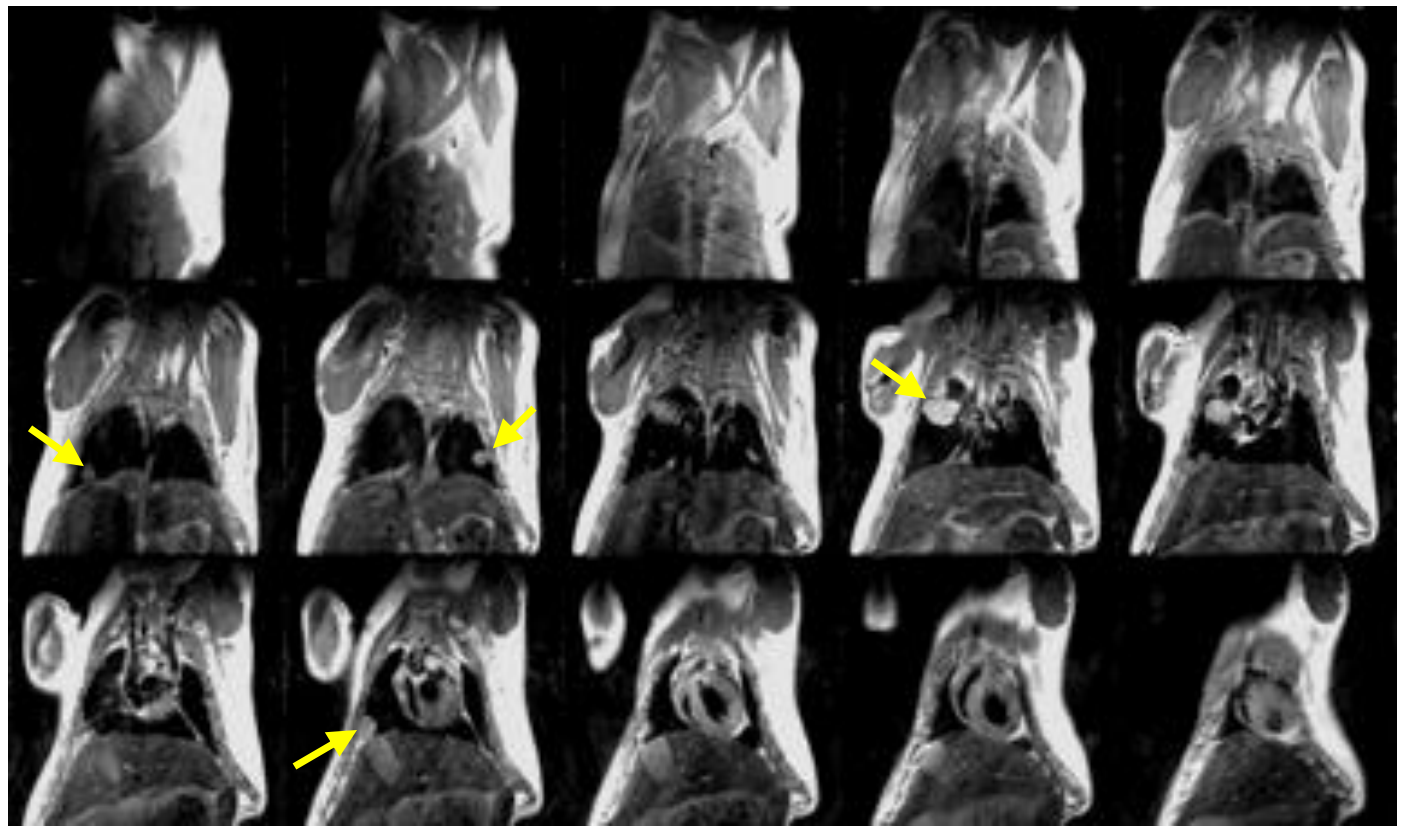

Figure 3-11. Representative spin echo MR images of a saline (top) and urethaneinjected (bottom) mouse 24 weeks following treatment. These scans were acquired approximately $30 \mathrm{~min}$ after gadolinium injection. Lungs tumors, as indicated by yellow arrows, are clearly visible in the urethane-treated mouse. In contrast, no lung tumors are detected in the saline-treated mouse. 
radioactivity counts. Furthermore, after $6 \mathrm{~h}$ approximately $1 \%$ of the injected dose remains in the blood circulation, minimizing potential signal contamination from ${ }^{64} \mathrm{Cu}-$ $\mathrm{Man}_{3}$-liposomes that may reside in the vasculature.

Figure 3-12A shows a coronally-acquired MRI revealing a tumor in the right lung (white arrow) and a PET image (Figure 3-12B) showing the distribution of $\mathrm{Man}_{3^{-}}$ liposomes $6 \mathrm{~h}$ post injection. Fused data sets (Figure 3-12C) confirm high tumor localization of the PET signal compared to normal lung tissue. Following the in vivo scans, the lungs were excised and imaged by PET without the spillover from the nearby liver. The distribution of DiO-labeled liposomes within the excised lung was evaluated by fluorescence imaging acquired using the IVIS Spectrum. This information could not be obtained in vivo due to limited tissue penetration and photon scattering. A photo of

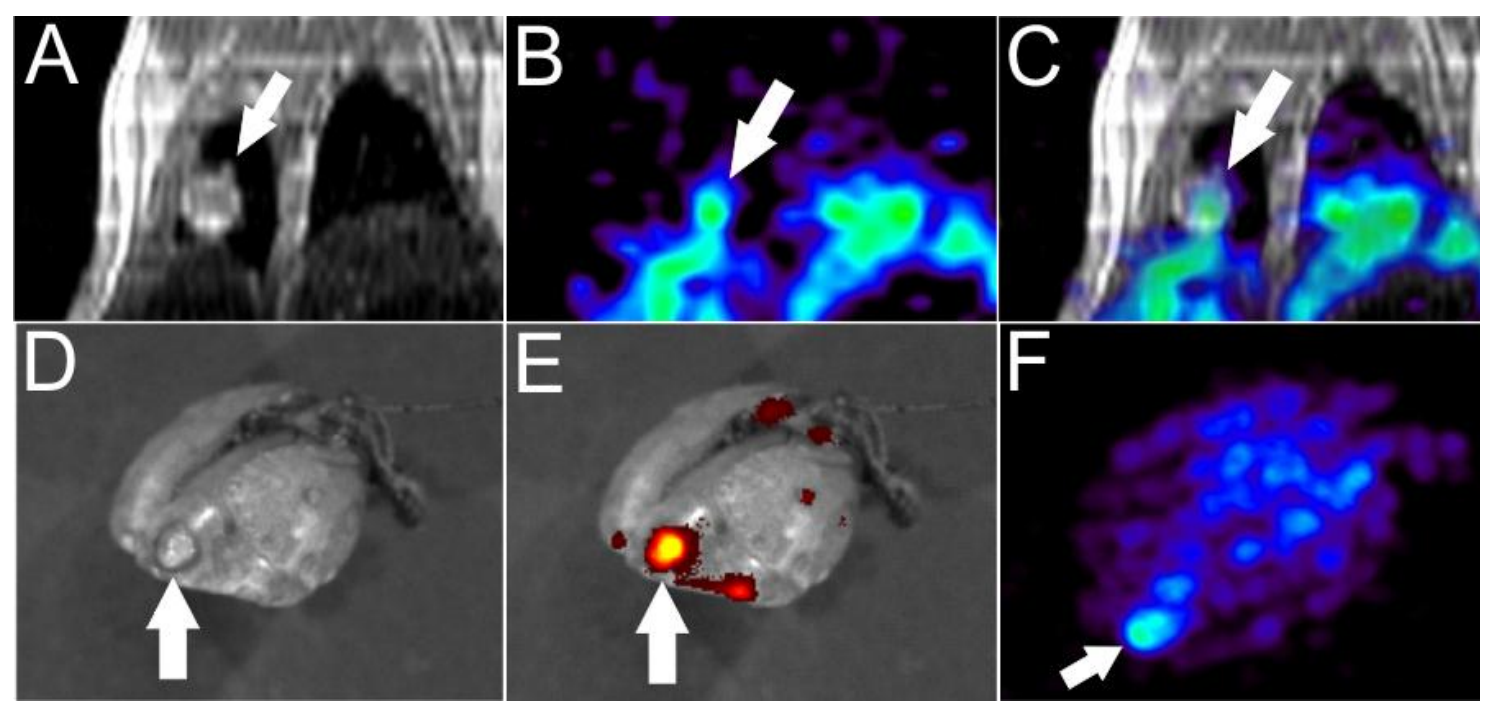

Figure 3-12. Representative in vivo images showing a lung tumor on coronal MRI (A) with enhanced ${ }^{64} \mathrm{Cu}$-labeled $\mathrm{Man}_{3}$-liposome uptake on PET $6 \mathrm{~h}$ after i.v. injection (B). PET-MR image registration (C) verifies tumor localization of the PET signal. Ex vivo fluorescence image of the lung was obtained to assess $\mathrm{DiO}$ distribution. A photo shows the tumor (D) which exhibited higher DiO accumulation compared to non-tumor lung areas. Ex vivo PET maximum intensity projection image $(\mathrm{F})$ showed focal ${ }^{64} \mathrm{Cu}$ signal in the area of the lung spatially corresponding to the tumor shown in the photograph. 
the excised lung clearly shows the tumor (arrow,

Figure 3-12D) as well as several other smaller tumors. The

fluorescence image

overlaid on the photo

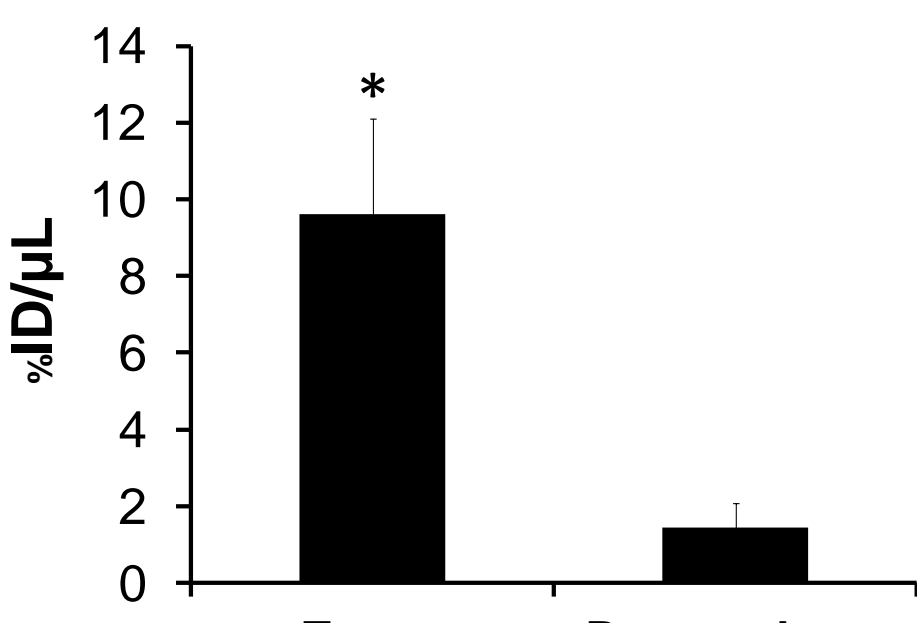

reveals a strong DiO-

fluorescent signal within

the tumor and minimal

accumulation in non-

tumor areas of the lung

\section{Tumor}

Figure 3-13. In vivo PET quantification of ${ }^{64} \mathrm{Cu}-\mathrm{Man}_{3}-$ liposome uptake measured in tumor and lung tissue at $6 \mathrm{~h}$ post injection. MRI-guided ROIs were drawn over tumors and areas in the lungs without visible tumor mass. The radioactivity concentration measured in tumors was corrected for PVE using a look-up table approach. RCcorrected ${ }^{64} \mathrm{Cu}-\mathrm{Man}_{3}$-liposome uptake $(\% \mathrm{ID} / \mu \mathrm{L})$ was $9.6 \pm$ 2.5 in tumor lesions compared to $1.4 \pm 0.6$ in normal lung tissue $(* P<0.05)$.

(Figure 3-12E). Ex vivo PET maximum intensity projection image, shown in Figure $3-12 \mathrm{~F}$, shows focal ${ }^{64} \mathrm{Cu}$ signal in the area of the lung spatially corresponding to the tumor and the fluorescence signal as shown in Figure 3-12, D-E.

The average diameter of lung tumors measured across all mice studied ranged from $1.25 \mathrm{~mm}$ to $2.65 \mathrm{~mm}$. Based on our look-up table approach, this corresponded to $\mathrm{RC}$ values ranging from 0.32 to 0.72 . As shown in Figure 3-13, the PV-corrected uptake of ${ }^{64} \mathrm{Cu}$-labeled $\mathrm{Man}_{3}$-liposomes in lung tumors was $9.62 \pm 2.49 \% \mathrm{ID} / \mu \mathrm{L}$ at $6 \mathrm{~h}$ post injection. In contrast, uptake in normal lung tissue was significantly lower $(1.4 \pm 0.6$ $\% \mathrm{ID} / \mu \mathrm{L})$ resulting in a tumor-to-normal lung ratio of 6.7 . 


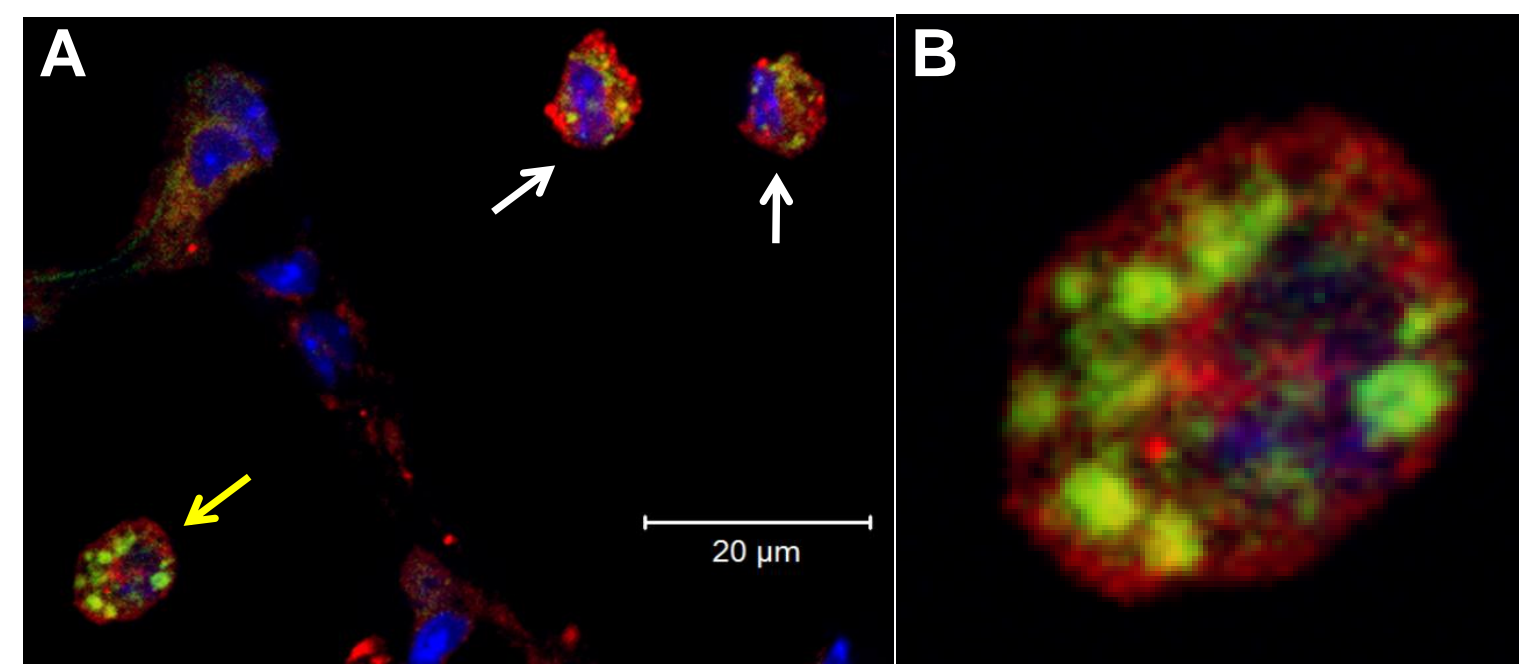

Figure 3-14. Confocal fluorescence microscopy revealed internalization of DiO-labeled $\mathrm{Man}_{3}$-liposomes (green) by $\mathrm{F} 4 / 80^{+}$macrophages (red) within the tumor stroma $6 \mathrm{~h}$ after i.v. liposome injection. Cell nuclei are stained with DAPI (blue). (A) A co-localized confocal image shows the intracellular localization of $\mathrm{Man}_{3}$-liposomes within TAMs. The enlarged view of the cell indicated by the yellow arrow is shown in $(\mathbf{B})$, clearly shows a clustered distribution of liposomes consistent with storage in macrophage endosomal structures.

Following PET imaging, the dual-labeled Man $_{3}$-liposome permitted a detailed assessment of cellular distribution by confocal fluorescence microscopy within lung tumor tissue. As shown in Figure 3-14A, significant DiO fluorescence was associated with F4/80-positive cells (TAMs). (In this image, the tumor was not captured in the field of view but was located immediately to the right.) Furthermore, this fluorescence was not limited to the circumference of TAMs, but rather was clustered within them as clearly seen on the enlarged view of the TAM (Figure 3-14B) indicated by the yellow arrow. This clustered distribution of $\mathrm{DiO}$ fluorescence is consistent with liposome entrapment in endosomal structures, signifying that tumor enhanced in vivo PET signals were the result ofMan $_{3}$-liposome-labeled TAMs. 


\subsubsection{Co-injection Results}

In order to accurately assess liposome lung distribution following co-injection, we first demonstrated that the fluorescence associated with each liposome type could be separated detected. As shown in Figure 3-16, the fluorescent dye DiO could be excited and detected apart from DiD using an excitation and emission filter pair of $500 \mathrm{~nm}$ and $540 \mathrm{~nm}$, respectively. Conversely, DiD could be detected apart from DiO using an excitation and emission filter pair of $605 \mathrm{~nm}$ and $640 \mathrm{~nm}$, respectively. This was expected based on their non-overlapping excitation and emission spectra (Figure 3-15).

Representative ex vivo fluorescence images of lungs $6 \mathrm{~h}$ after the co-injection of $\mathrm{Man}_{3}$ and plain liposomes as well as $\mathrm{Man}_{3}$ and PEG liposomes are shown in Figure 3-17A, and Figure 3-17B, respectively. From these images it was clearly evident that $\operatorname{Man}_{3}$ and plain liposomes strongly localized at lung tumors, however, $\operatorname{Man}_{3}$ liposomes exhibited a lower background signal in non-tumor lung tissue. PEG liposomes showed a diffuse lung distribution and consequently poor tumor contrast likely due to their enhanced blood circulation time. To examine uptake in various tissues, we computed uptake ratios between the tumor and remote lung, tumor and liver, and tumor and spleen for each liposome type based on the fluorescence images. Tissue ratios were computed to avoid potential biases in the excitation of the fluorescent dyes as well as to avoid biases due to differences in signal attenuation in animal tissues. As shown in Figure 3-18A, Man 3 -liposomes exhibited a higher tumor-to-remote lung and tumor-to-spleen ratio compared to plain liposomes. However, the tumor-to-liver ratio was comparable. $\mathrm{Man}_{3}$-liposomes also exhibited a high tumor-to-remote lung ratio when co-injected with PEG liposomes. PEG liposomes exhibited the lowest tumor-to-remote lung ratio of the 3 
A

Excitation Spectra

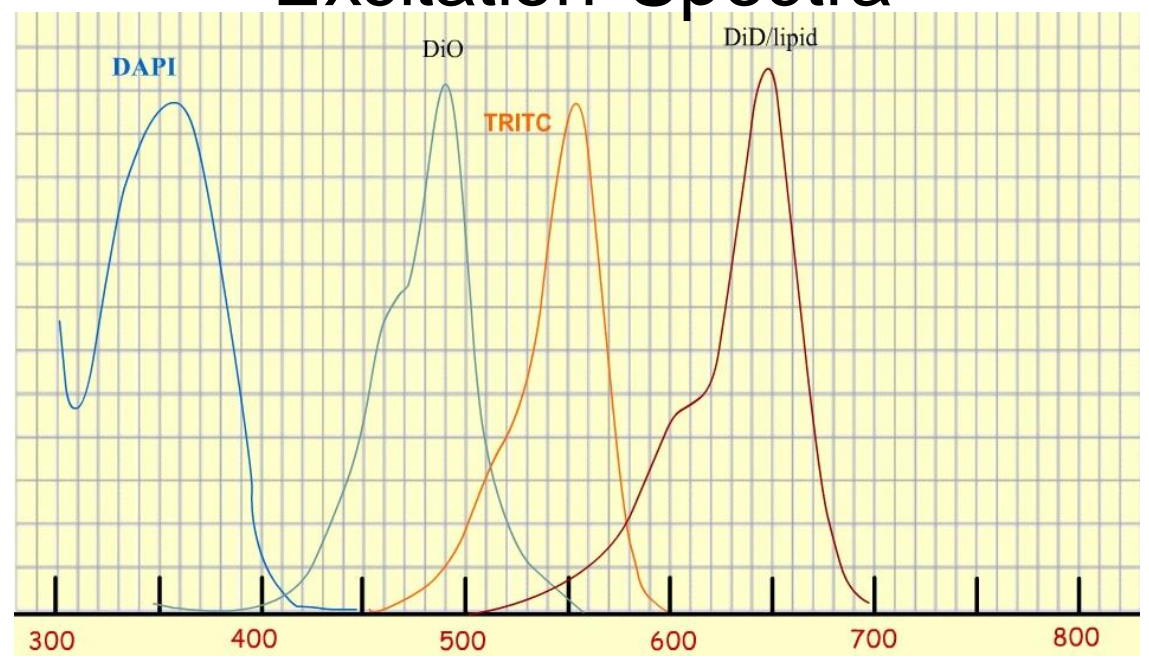

B

Emission Spectra

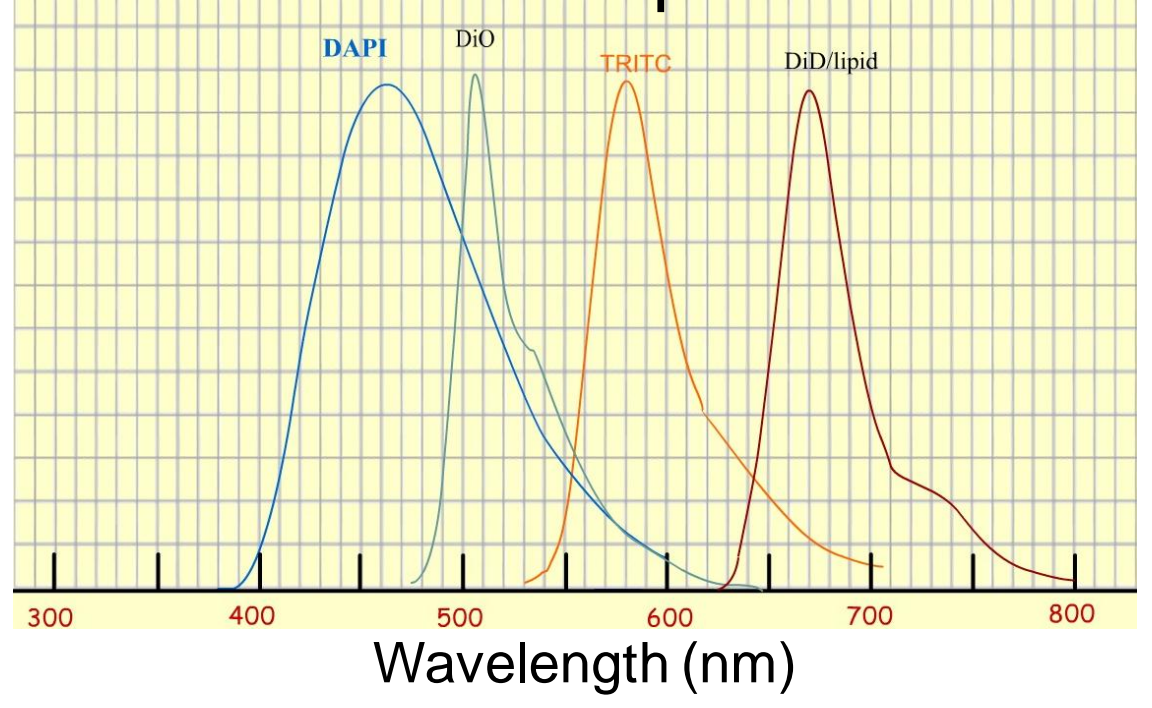

Figure 3-15. Excitation (A) and emission (B) spectra for each fluorescent dye used for the analysis of liposome uptake by TAMs by confocal microscopy. DiO and DiD are the fluorescent dyes used to label liposomes. DAPI is used to visualize cell nuclei and TRITC is the dye conjugated to the secondary antibody used for staining the anti-mouse F4/80 primary antibody. 
liposomes studied. This is likely due to the slow rate of blood clearance of these liposomes which results in a strong background signal, particularly in the wellvascularized tissue of the lung. PEG liposomes also showed a reduced tumor-to-liver ratio, consistent with a lower rate of capture by the reticuloendothelial system.

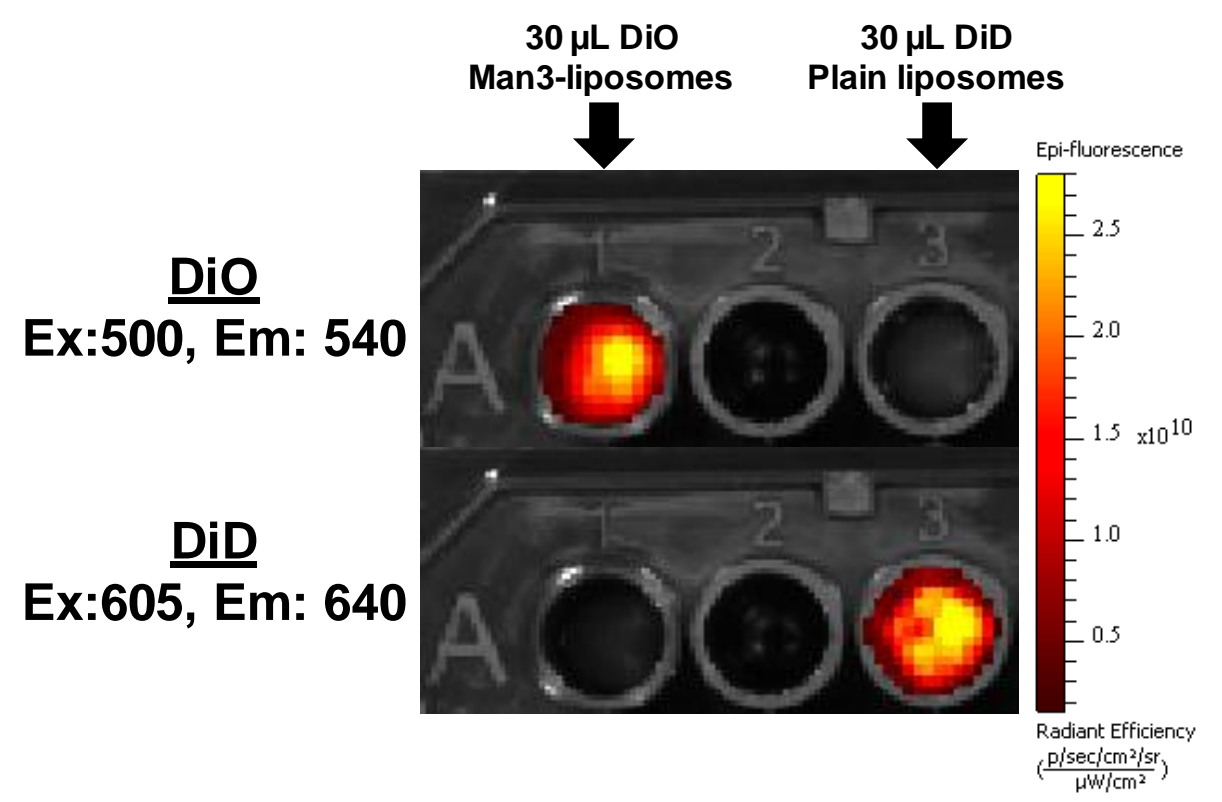

Figure 3-16. A preliminary well plate study demonstrating that $\mathrm{DiO}$ and $\mathrm{DiD}$ fluorescence associated with different liposome types can be detected separately. The top row shows that DiO-labeled $\mathrm{Man}_{3}$-liposomes could be imaged apart from DiD using an excitation and emission filter pair of $500 \mathrm{~nm}$ and $540 \mathrm{~nm}$, respectively. Likewise, the bottom row shows that DiD-labeled plain liposome could be imaged apart from $\mathrm{DiO}$ using an excitation and emission filter pair of $605 \mathrm{~nm}$ and $640 \mathrm{~nm}$, respectively. This studied confirms that following their co-injection, liposomes can be imaged separately allowing their biodistribution to be uniquely tracked.

Finally, we used confocal microscopy to visualize liposome uptake by TAMs at the cellular level. As shown in Figure 3-19A, $\mathrm{Man}_{3}$ and plain liposomes, indicated by green and yellow fluorescence, respectively, were both internalized by $\mathrm{F} 4 / 80^{+}$cells upon their co-injection. Similarly, there appeared to be equal uptake by F4/80 cells of $\mathrm{Man}_{3}$ and PEG liposomes following their co-injection (Figure 3-19B). This result is unexpected considering the ex vivo images of the PEG liposome lung distribution showed 
no evidence of accumulation within tumor areas. Based on this study, it appears that localization of PEG liposomes within TAMs may occur and that tumor contrast on whole-lung fluorescent images might have been improved by allowing its blood clearance to reach levels comparable to $\mathrm{Man}_{3}$-liposomes. Finally, it is also interesting that for both co-injection studies, $\mathrm{DiO}$ and $\mathrm{DiD}$ fluorescence within a given macrophage appears to be localized to common endosomes.

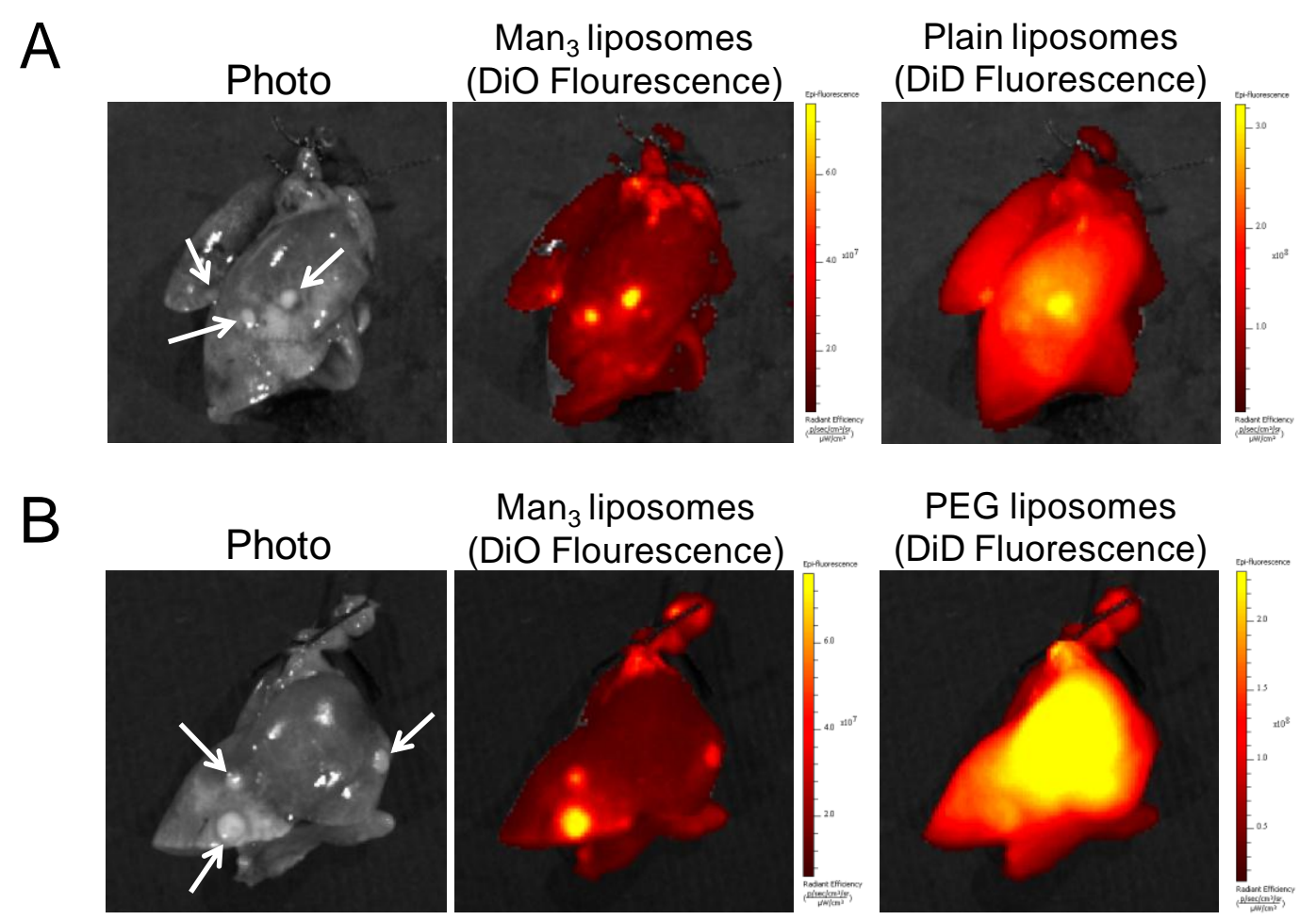

Figure 3-17. Representative photos and fluorescent images from the liposome coinjection study. Images of excised lungs $6 \mathrm{~h}$ after the co-injection of $\mathrm{Man}_{3}$ and plain liposomes (A) and $\mathrm{Man}_{3}$ and PEG liposomes (B). Strong fluorescence signal associated with $\mathrm{Man}_{3^{-}}$and plain liposomes is localized to lung tumors (identified by white arrows on the photo). However, compared to $\mathrm{Man}_{3}$-liposomes, plain liposomes exhibit a higher background signal. PEG liposomes show a diffuse lung distribution and consequently poor tumor contrast likely due to their enhanced blood circulation time. 


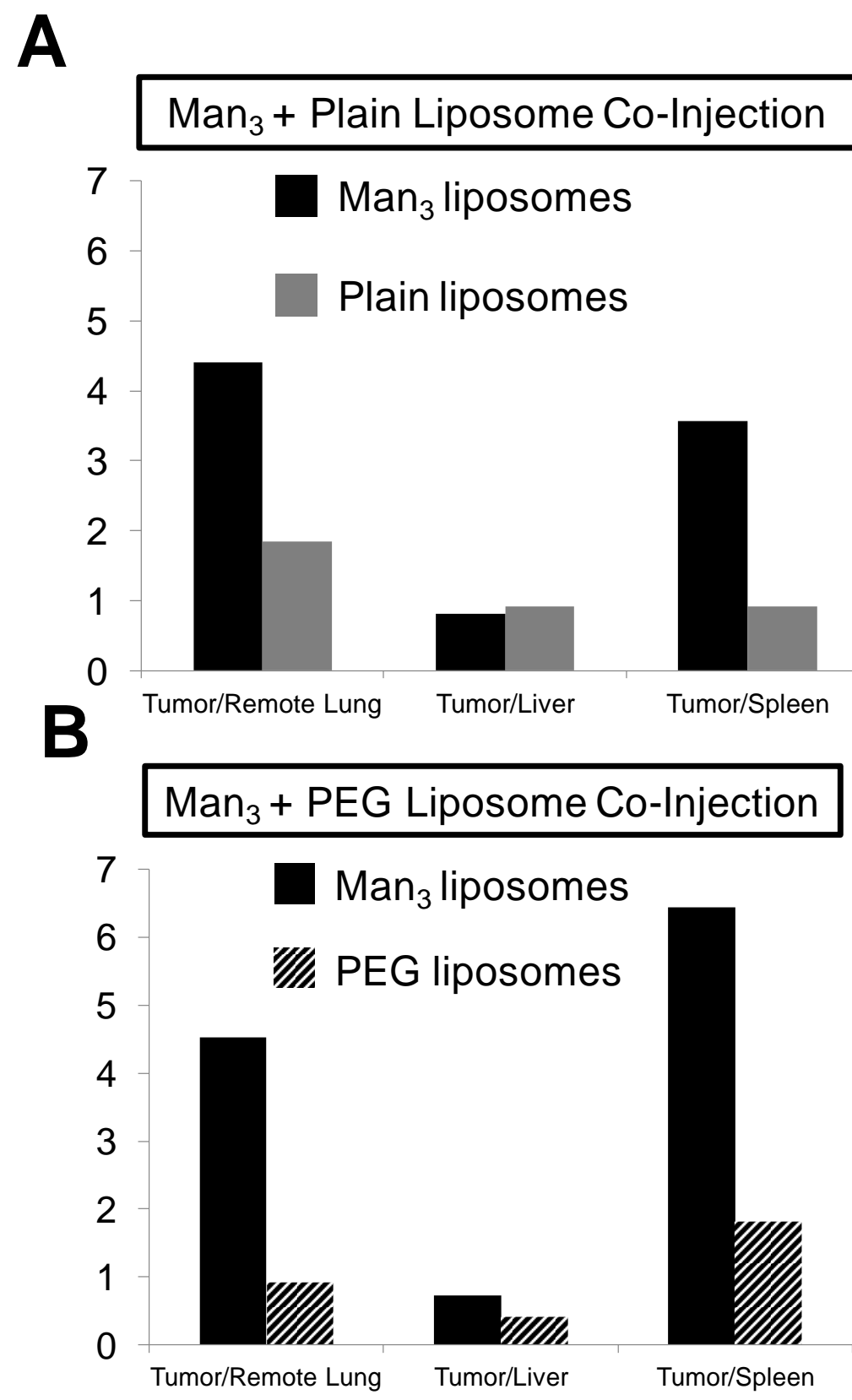

Figure 3-18. Tumor-to-tissue ratios measured from fluorescence images of harvested organs following the co-injection of $\mathrm{Man}_{3}$ and plain liposomes (A) and $\mathrm{Man}_{3}$ and PEG liposomes (B). Man 3 -liposomes exhibited a higher tumor-to-remote lung and tumor-to-spleen ratio compared to plain liposomes, while tumor-to-liver ratios were comparable. $\mathrm{Man}_{3}$-liposomes also exhibited a high tumor-to-remote lung ratio following co-injection with PEG liposomes, likely due to the slow rate of blood clearance of PEG liposomes. PEG liposomes also showed a reduced tumorto-liver ratio, consistent with a lower rate of capture by the RES. 


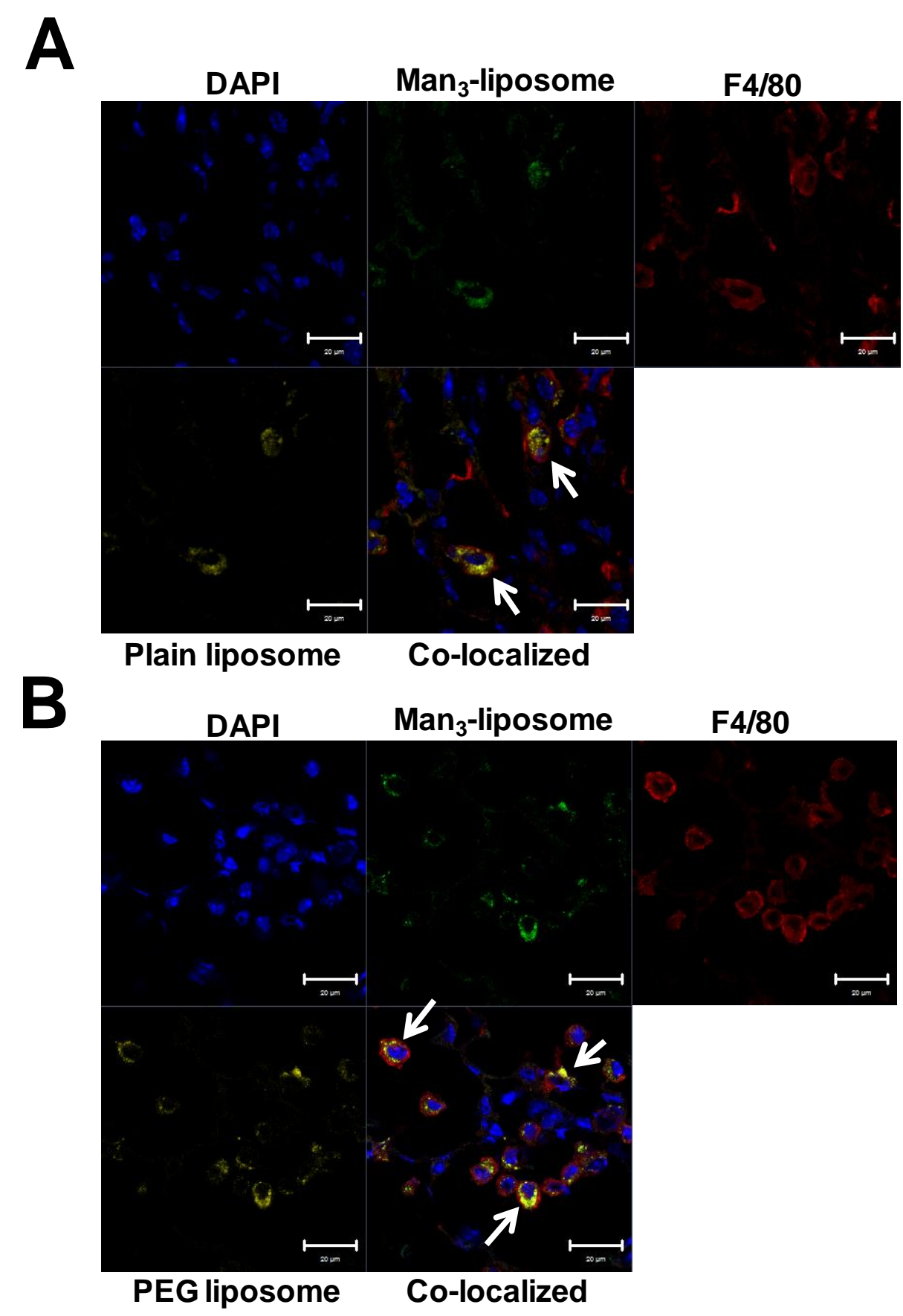

Figure 3-19. Confocal images of lung tumor stroma following liposome co-injection. Following their co-injection, $\mathrm{Man}_{3}$ and plain liposomes, indicated by green and yellow fluorescence, respectively, were both internalized by $\mathrm{F} 4 / 80^{+}$cells (A). Similarly, $\mathrm{Man}_{3-}$ and PEG liposomes were also internalized by $\mathrm{F} 4 / 80^{+}$cells following their coinjection (B). The spatial distribution of each dye within a given $\mathrm{F} 4 / 80^{+}$cell appears to be identical, suggesting entry into cells by common surface receptors. 


\subsection{DISCUSSION}

Recent evidence has highlighted the role of macrophages, particularly of the M2 subtype, in the malignant progression of lung tumors. This recognition has spurred considerable effort to establish techniques to quantitatively monitor TAMs through noninvasive imaging. It has been previously shown that incorporating mannosylated phospholipids into liposomes improves targeting to peritoneal macrophages in vivo by receptor-mediated endocytosis $[72,73]$. This approach for monitoring TAMs by PET imaging has two significant advantages over fluorescently-labeled iron oxide nanoparticles designed for detection by MRI and optical techniques. First, the targeted liposomes are radiolabeled and thus are able to be tracked using PET imaging, which possesses a higher intrinsic sensitivity $\left(10^{-8}\right.$ to $10^{-9} \mathrm{~mol} / \mathrm{L}$ for PET compared to $10^{-3}$ to $10^{-}$ ${ }^{5} \mathrm{~mol} / \mathrm{L}$ for MRI). Second, liposomes provide a flexible platform for delivering both hydrophobic and hydrophilic cargo. For this study the aqueous interior core of liposomes was utilized to encapsulate a hydrophilic chelating agent to allow for remote labeling of the PET radionuclide, ${ }^{64} \mathrm{Cu}$. This compartment of the liposome also could be used to encapsulate a wide array of hydrophilic drugs including cytotoxic agents that abolish TAMs through apoptosis or immunomodulatory agents that could trigger their reversal from a pro-tumoralM2 phenotype to a tumoricidalM1 phenotype. For example, it was recently shown that the cytokine IFN- $\gamma$ induced human TAMs to switch from immunosuppressive to immunostimulatory phenotype [96].

Results from our in vitro studies revealed a significantly higher association of Man $_{3}$-liposomes with MØ(IL-4/13) compared with plain liposomes. Furthermore, MØ(IL-4/13) exhibited significantly higher uptake of $\mathrm{Man}_{3}$-liposomes compared to 
MØ(LPS/IFN- $\gamma)$, correlating with CD206 expression on these cells. Interestingly, MØ(LPS/IFN- $\gamma$ ) exhibited a preference for $\mathrm{Man}_{3}$-liposomes over plain liposomes in spite of their low expression of CD206. This result may be explained by a study that found that IFN- $\gamma$ enhances mannose receptor-mediated phagocytosis, despite its effects on surface mannose receptor down-regulation [95]. More studies are needed to determine what receptors are responsible for the uptake of $\mathrm{Man}_{3}$-liposomes by MØ(LPS/IFN- $\left.\gamma\right)$ and also if this uptake behavior occurs in vivo.

By PET imaging, we showed that $\mathrm{Man}_{3}$-liposomes exhibited enhanced signal in regions corresponding with lung tumors compared to areas of remote lung at $6 \mathrm{~h}$ post injection. Using a look-up table approach, we estimated the RC value for each tumor studied in order to correct for PVE due to their small size. We acknowledge some limitations to this approach. First, we did not account for radioactivity "spill-in" of surrounding tissue into tumor ROIs. We believe that the size of the lesion is the primary determinant of the RC, with background activity influencing it only to a small degree. This has been demonstrated by other groups with phantom experiments [97]. Furthermore, as shown by in vivo and ex vivo PET images of the same lung, ROI-derived radioactivity concentrations of lung tumors were not significantly affected by their proximity to the liver. Another limitation of this approach is that we do not account for respiratory motion which can cause image blurring particularly in the thoracic region. Studies in mice have shown that non-respiratory gated PET imaging can lead to significant tumor standardized uptake value (SUV) underestimations depending on lesion size [98]. However, a gated PET acquisition typically suffers from a reduced signal-tonoise ratio compared to a non-gated one and because of this we chose not to employ it. 
With regard to lung distribution, there were distinct differences between $\mathrm{Man}_{3}$ and plain-liposomes following their co-injection. The fluorescence signal of plain liposomes was significantly higher than that of $\mathrm{Man}_{3}$-liposomesin areas of lung absent of visible tumors. This finding is likely not due to tissue autofluorescence because DiD excitation occurs within a range of wavelengths where tissue autofluorescence is minimal. A possible explanation for the elevated remote lung signal is a delayed lung clearance of plain liposomes compared to $\mathrm{Man}_{3}$-liposomes. However, this is not likely since their measured blood clearance half-lives were nearly identical. Another possible explanation for this behavior could be due to an increased uptake of plain liposomes by resident alveolar macrophages. While this explanation is not consistent with other studies that have demonstrated enhanced targeting to alveolar macrophages by mannose-coated liposomes after intratracheal administration in rats [91] and i.v. administration in mice [99], it is unknown if lung tumors influence the phagocytic behavior of these cells. Lastly, the compliment system is a major dominant factor in the clearance of liposomes [100]. Additional studies would be needed to determine whether plain liposomes more efficiently activate complement, which could promote better recognition by alveolar macrophages and lead to higher lung sequestration. A flow cytometry study could be designed to help determine if lung resident macrophages are responsible for uptake or if the delayed clearance of plain liposomes is due to mechanisms not related to cellular uptake.

Our results obtained from confocal microscopy verified that macrophages were responsible for the elevated fluorescence signal observed within the areas of lung tumors on whole-lung fluorescent images. However, the cellular uptake patterns of $\mathrm{Man}_{3}$ and 
plain liposomes were unexpected. The clustered distribution of $\mathrm{DiO}$ and $\mathrm{DiD}$ fluorescence within a given TAM appeared to be co-localized, suggesting that the inclusion of mannose ligand did not mediate better liposome recognition and subsequent macrophage internalization relative to plain liposomes despite a great majority of these cells being CD206 ${ }^{+}$. Confocal microscopy images also revealed that PEG liposomes were internalized by TAMs, a finding that was disguised on whole-lung fluorescence images due to their slow rate of clearance from the blood and prominent signal throughout the lung. These findings are significant because they indicate that $\mathrm{Man}_{3^{-}}$ liposomes, which are rapidly cleared from the blood relative to PEG liposomes, are able to target lung TAMs and that a slow rate of blood clearance is not required for targeting these cells. Rapid blood clearance and effective targeting are attractive properties of $\mathrm{Man}_{3}$-liposomes and highlight their potential use as TAM imaging and/or delivery agents. Our data show that $\mathrm{Man}_{3}$-liposomesnot only demonstrated targeting of TAMs, but also rapid clearance in normal lung which is critical for achieving high tumor contrast by PET imaging.

\subsection{CONCLUSION}

TAMs represent a unique opportunity to impede tumor progression. Their influence on tumor growth has been shown by numerous groups to be dependent on their differentiation state. In this chapter we demonstrated the successful targeting of mannosylated liposomes to TAMs in a mouse model of pulmonary adenocarcinoma. Confocal microscopy verified liposome internalization by TAMs implicating that focal

${ }^{64} \mathrm{Cu}$ accumulation in areas of lung tumors observed on PET images was due to TAMmediated uptake of the targeted liposomes. Although co-injection studies revealed that 
plain and PEG liposomes are both internalized by TAMs, Man 3 -liposomes exhibited the highest tumor to remote lung ratios on whole-lung fluorescent images, implicating it as a promising agent for imaging TAMs in the setting of lung cancer. 


\section{DISSERATION CONCLUSIONS}

Inflammation, both local and systemic, is a hallmark of many important pulmonary diseases and has become a potential therapeutic target. However, the mechanisms that underlie persistent inflammation remain unclear and we currently lack sensitive measurement techniques that can directly report on leukocyte trafficking in the lung. Traditional imaging techniques can provide information regarding lung anatomy and function, but can give no information on inflammatory processes or can they monitor the activity of infiltrated leukocytes. The development of molecular imaging probes that recognize specific molecular markers on leukocytes or report on their activation state is an important goal and is an area of active research. For this dissertation I explored two different noninvasive, molecular imaging strategies for monitoring lung neutrophilic inflammation based on positron emission tomography imaging in mice.

Having first established a noninvasive method for deriving the blood input function in mice directly from gated PET images [39], I explored three-compartment model analysis of lung FDG uptake to quantitatively assess neutrophil metabolic activity in a mouse model of acute lung inflammation. The appeal of this method lies in its noninvasive and quantitative approach in measuring pulmonary FDG uptake by neutrophils in mice: a surrogate marker for neutrophil activation. In this model, I measured a 2.24-fold increase in FDG uptake $\left(\mathrm{K}_{\mathrm{i}}\right)$ in the lungs compared to non-infected controls. Importantly, the mean lung $\mathrm{K}_{\mathrm{i}}$ values computed here were not significantly different from previously reported lung values for mice of similar age and background strain computed from input functions obtained by arterial sampling. Finally, utilizing the same image-derived blood input function, selective slices of the PET image data were 
transformed into parametric maps of local $\mathrm{K}_{\mathrm{i}}$ which provided spatial information regarding local neutrophilic inflammation not obtained when lung image analysis is performed on a regional level.

Because the PET-measured FDG signal may be the result of uptake by malignant cells as well as other cell types found in the lung, we also explored an alternative imaging method for neutrophil monitoring based on the peptide cFLFLF. This peptide was previously reported to be an antagonist to the neutrophil FPR, yet its development was stalled due to poor imaging characteristics. We demonstrated that the bioavailability and blood clearance properties of the peptide ligand could be improved by PEG conjugation without adversely affecting its binding affinity or stimulatory properties towards the FPR on neutrophils. Quantification of PET images $18 \mathrm{~h}$ after peptide intravenous injection in K. pneumoniae-challenged and control mice yielded average lung SUVs of $0.142 \pm 0.054$ and $0.028 \pm 0.003$, respectively $(P<0.003)$. Our results suggest that this peptide may be suited to image neutrophils in vivo and could play an important role in evaluating future ant-inflammatory therapeutics designed to inhibit lung neutrophil recruitment and/or function in the preclinical setting. Furthermore, because ${ }^{64} \mathrm{Cu}$ is a PET radionuclide with a moderate rate of radioactive decay, we posit that this peptide may have translational potential to humans.

In addition to neutrophils, macrophages participate centrally in many common lung diseases ranging from COPD to acute respiratory distress syndrome. Furthermore, recent studies have emerged identifying macrophages as critical components in the growth and spread of lung tumors. Chapter 3 of this dissertation examined the targeting of TAMs using liposomes surface coated with a mannose ligand. This liposome modification has 
been shown to improve recognition by M2 macrophages and cellular entry by mannose receptor-mediated endocytosis but has not been studied as a macrophage targeting agent for PET imaging. Initial studies conducted in vitro confirmed that $\mathrm{Man}_{3}$-liposomes were taken up nearly 2-fold higher by M2 (IL4/13-stimulated) bone marrow-derived macrophages compared to M1 (LPS/IFN- $\gamma$-stimulated) cells. Furthermore, using flow cytometry we showed that IL4/13-stimulated cells exhibited the highest levels of mannose receptor (CD206), which correlated well with Western blotting. In vivo studies were conducted using a urethane-induced lung tumor mouse model. In vivo PET imaging with radiolabeled $\mathrm{Man}_{3}$-liposomes revealed high tumor localization of Man-liposomes 6 $h$ following intravenous injection. PET image quantification revealed that $\mathrm{Man}_{3^{-}}$ liposomes had an increased accumulation in tumor tissue compared to normal lung tissue $(9.62 \pm 2.49$ vs. $1.4 \pm 0.6 \% \mathrm{ID} / \mu \mathrm{L}, P<0.05)$, strongly correlating with the relative densities of $\mathrm{F} 4 / 80^{+}$macrophages estimated in these tissues by immunohistochemistry. Subsequent ex vivo lung biodistribution and confocal microscopy studies confirmed

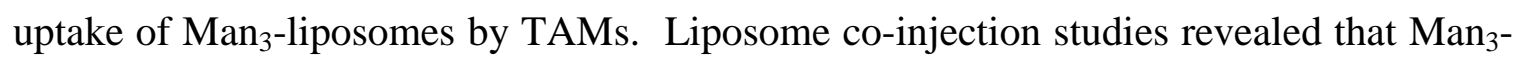
liposomes exhibited the highest tumor contrast relative to normal lung tissue at the time point studied. This contrast appears to be due to rapid clearance of Man 3 -liposomes in normal lung as confocal analysis of tissue at lung tumor boundaries revealed near equivalent uptake of both $\mathrm{Man}_{3}$-liposomes and plain liposomes by common TAMs. 


\section{FUTURE RESEARCH}

Utilizing a mouse model of acute lung inflammation, we demonstrated significant lung accumulation of the modified peptide cFLFLF in vivo relative to normal lungs [36]. However, these studies do not definitively show that the enhanced accumulation of the intravenously-injected peptide in inflamed lung tissue measured from the PET images is a result of binding to infiltrated neutrophils. Recently, our research group published on the successful labeling of the peptide with the near infrared fluorophore, Cyanine 7 [101]. Using this peptide labeling strategy, a flow cytometry-based study could be conducted to quantify the association of the peptide with various leukocyte populations including neutrophils and macrophages that are present in the lung after $K$. pneumonia-challenge. Additionally, a future study could incorporate a scrambled version of the peptide to address neutrophil specificity. Both in vitro neutrophil binding studies as well as in vivo imaging studies could be carried with the scrambled and unaltered peptide for comparison.

For the inflammatory lung model used in this study, the optimum imaging time was determined to be $18 \mathrm{~h}$. However, the stability of the ${ }^{64} \mathrm{Cu}$ label to the peptide over time has yet to be studied. The biodistribution data shown in Figure 2-3 shows high concentrations of radioactivity in the liver, kidney, and small intestine. These organs are not typically sites of neutrophil clearance; therefore more studies are needed to determine the proportion of radioactivity that is neutrophil-bound versus that which is free ${ }^{64} \mathrm{Cu}$.

In Chapter 3, successful targeting of $\mathrm{Man}_{3}$-liposomes to TAMs in vivo was demonstrated by PET and fluorescence imaging, providing a proof of concept for the use of these nanoparticles to image and/or potentially delivery therapeutic drugs to this 
macrophage population. Although limited in scope, our liposome co-injection data suggests that plain and PEG liposomes target TAMs with equal efficiency to $\mathrm{Man}_{3^{-}}$ liposomes. To rule out the possibility that the uptake of plain and PEG liposomes by TAMs was influenced by the presence of $\mathrm{Man}_{3}$-liposomes, plain and PEG liposomes could be injected separately. Furthermore, an experiment could be easily conducted where $\mathrm{Man}_{3}$-liposome internalization by TAMs is studied following their co-injection with excess amounts of free mannose. This study would be useful for assessing the role

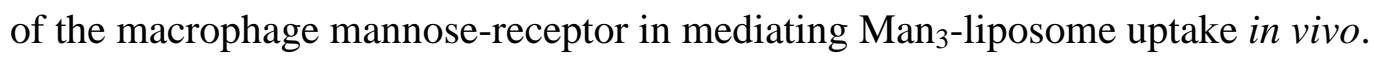

It is likely that through modifications of their physical properties, Man 3 -liposomes can achieve a higher binding affinity for the mannose receptor, thus facilitating better recognition and internalization by TAMs. The liposomes used in this study were composed of PC, a neutral lipid. However, liposome composition can be modified to achieve more efficient targeting in certain cases. For example, more efficient lung retention and macrophage phagocytosis has been shown to be achieved with the inclusion of the negatively charged phosphatidylserine (PS). A disadvantage of constructing liposomes with PS is a reduction in liposome stability in the presence of serum [102]. In addition to composition, liposome size also plays a role in lung retention [103]. Although larger liposomes achieve higher lung retention, this must be balanced against the size limitation for which particles can achieve extravazation into tumor interstitium and the rate of which they are cleared from the blood.

Liposomes with an increased surface density of the mannosylated phospholipid may show an improved affinity towards the mannose receptor, but the consequence of such a design in vivo is unknown. It is likely that the limiting factor for targeting lung 
TAMs by i.v. injection is not due to TAM recognition but rather the number of liposomes that can extravagate into the tumor before being significantly removed from the blood by hepatic Kupffer cells.

As a result of the observation that PEG liposomes are internalized by TAMs, a potential liposome construct is to tether mannose structures to the distal end of PEG. Such a design may offer two attractive properties: effective mannose-receptor recognition and a prolonged circulation time by limiting binding of serum complement proteins [104]. A longer blood circulation time may increase the opportunity for liposomes administered systemically to leak out of the vascular compartment and interact with TAMs via the EPR effect. However, depending on the uptake mechanism, protecting liposomes from being captured by liver Kupffer cells through PEGylation may also impair efficient uptake by TAMs. Furthermore, some studies have shown that PEGylation of liposomes does not improve lung retention times, therefore this strategy must be carefully considered [105].

Another factor that strongly dictates liposome blood clearance and tissue distribution is the route of administration. The tail vein was chosen as the route of liposome administration in this study because it allowed liposomes to flow through the lung capillary bed before encountering the liver. However, the intravenous route will always be limited by rapid liver uptake and destruction by serum components. Liposome delivery through the airways is a potential route that may improve lung retention times. Studies have demonstrated successful delivery of mannosylated liposomes to alveolar macrophages in rats via intratracheal delivery [92]. However, it is currently unknown if successful TAM targeting can be achieved by this route of delivery. 
One of the major advantages of liposomes is that they can function as a drug delivery vehicle in addition to an imaging agent. The liposome mediated intracellular delivery of clodronate has been shown to effectively deplete macrophages associated with a variety of tumor models [59]. A future study could compare the efficiency of TAM depletion of clodronate-encapsulated plain vs. Man $_{3}$-liposomes in the urethane lung tumor model. Lung tumor growth rates in each group could be tracked over time using MRI. 


\section{APPENDIX A: Image-Derived Input Function Validation}

The purpose of this study is to demonstrate that the blood input function, required for the quantification of FDG uptake by compartmental modeling, can be accurately and noninvasively measured from OSEM-MAP reconstructed, cardiac-gated PET images. We validate this by comparing FDG blood radioactivity concentrations measured by left ventricular blood pool (LVBP) ROIs to physical blood samples taken during PET scanning. Additionally, we measured the FDG influx rate $\left(\mathrm{K}_{\mathrm{i}}\right)$ in myocardial tissue and compared it to reported $\mathrm{K}_{\mathrm{i}}$ values in literature which were computed based on physicallysampled input functions in mice of similar age and background strain.

\section{Materials and Methods}

PV effects in PET lead to quantitative under-estimations of the regional concentrations of radioactivity in reconstructed images and corresponding errors in compartmental model kinetic parameters. Because of the relatively small dimension of the murine ventricle, radioactivity measurements in the LVBP suffer from PV effects. To estimate the magnitude of the PV effect in the LVBP and in the myocardium, a Jaszczak Micro Deluxe hot rod phantom (Data Spectrum Corporation, Hillsborough, North Carolina) with interior rods of various diameters $(1.2,1.6,2.4,3.2,4.0,4.8 \mathrm{~mm})$ was filled with a known concentration of FDG. PET was acquired and an image of the phantom was reconstructed using OSEM-MAP algorithm. For comparative purposes, we also reconstructed the image using Filtered Back-Projection (FBP) reconstruction. In both reconstructions, the images were subjected to a CT-based attenuation correction. The average ROI-derived radioactivity concentrations measured for the different sized rods were divided by the true activity concentration to obtain a recovery coefficient (RC) 
curve spanning the range of rod sizes. A unique RC curve was generated for each reconstruction type. RCs for the LVBP and myocardium were estimated from the RC curves and applied to time-activity curve measurements.

Five non-fasted C57/BL6 mice (25-30 g) were used in this study. PET and CT images were acquired and reconstructed using the same protocols described in 1.2.2 and 1.2.3, respectively. Circular ROIs approximately 3 pixels $(1.2 \mathrm{~mm})$ in diameter were manually drawn on contiguous transverse planes in the area corresponding to the LVBP in which the LV cavity was visible. These ROIs, which were drawn on the last time frame, were projected onto all previous time frames to obtain blood pool TACs. Finally, the TAC measured from each slice was averaged together to yield the IDIF. Cardiac gating permitted the drawing of LVBP ROIs when the phase of the cardiac cycle was at near-diastole, thus minimizing contamination into the LVBP by spillover of radioactivity from the surrounding myocardium. To correct for PV effects in the LVBP, the IDIF was boosted by the RC value estimated from the RC curve (Figure 6-1).

Physical blood samples were obtained at 43 and 56 minutes post FDG administration via a small incision in the distal tail while the animal was being PET scanned. Approximately $40 \mu \mathrm{L}$ of blood was collected in a pre-weighed capillary tube for each time point. Excessive bleeding was prevented by applying gentle pressure for approximately $1 \mathrm{~min}$. Each blood sample was weighed using an analytical balance and the associated radioactivity counted in a well counter previously used to calibrate the PET scanner. Radioactivity concentrations (in $\mu \mathrm{Ci} / \mathrm{g}$ ) of each blood sample were determined by dividing the decay-corrected radioactivity measured in the well counter by the net weight of the blood in the capillary tube. Bland-Altman analysis was used to 
assess the agreement between image-derived and sampled blood radioactivity concentrations [106].

To further validate the accuracy of the IDIF, the net FDG influx constant $\left(\mathrm{K}_{\mathrm{i}}\right)$ was computed in the myocardium by kinetic analysis. Kinetic transport rate constants of FDG uptake $\left(\mathrm{k}_{1}, \mathrm{k}_{2}\right.$, and $\left.\mathrm{k}_{3}\right)$ in the myocardium were estimated by the three-compartment FDG model using non-linear regression as discussed in 1.2.4. From these estimated rate constants, $\mathrm{K}_{\mathrm{i}}$ was computed (based on Eq. 3) in the heart and compared to values obtained by physical blood sampling previously reported in the literature.

\section{Results}

Figure 6-1 shows OSEM-MAP and FBP reconstructed PET images of the hot rod phantom and the RC curves derived from them. From these curves and a previously obtained MRI measurement of $3.3 \pm 0.2 \mathrm{~mm}$ for the internal LV diameter, an RC value of 0.92 was determined from the LVBP for OSEM-MAP based reconstructions. This measurement is in close agreement with a previously published value measured by ultrasound in mice of the same age and background strain [107]. The value of the RC correction factor was expected to approach unity because the diameter of the mouse LV chamber is approximately twice the FWHM spatial resolution of the Focus 120 PET scanner $[108,109]$. Other studies have demonstrated peak signal recoveries of $86 \%$ for spherical structures that are twice the FWHM, consistent with our findings [109]. The $\mathrm{RC}$ for the myocardium at end diastole was estimated to be 0.28 (based on a thickness of $0.9 \pm 0.1 \mathrm{~mm}$ previously measured by MRI). For FBP reconstructions, the RC for the LV cavity was estimated to be 0.57 . 

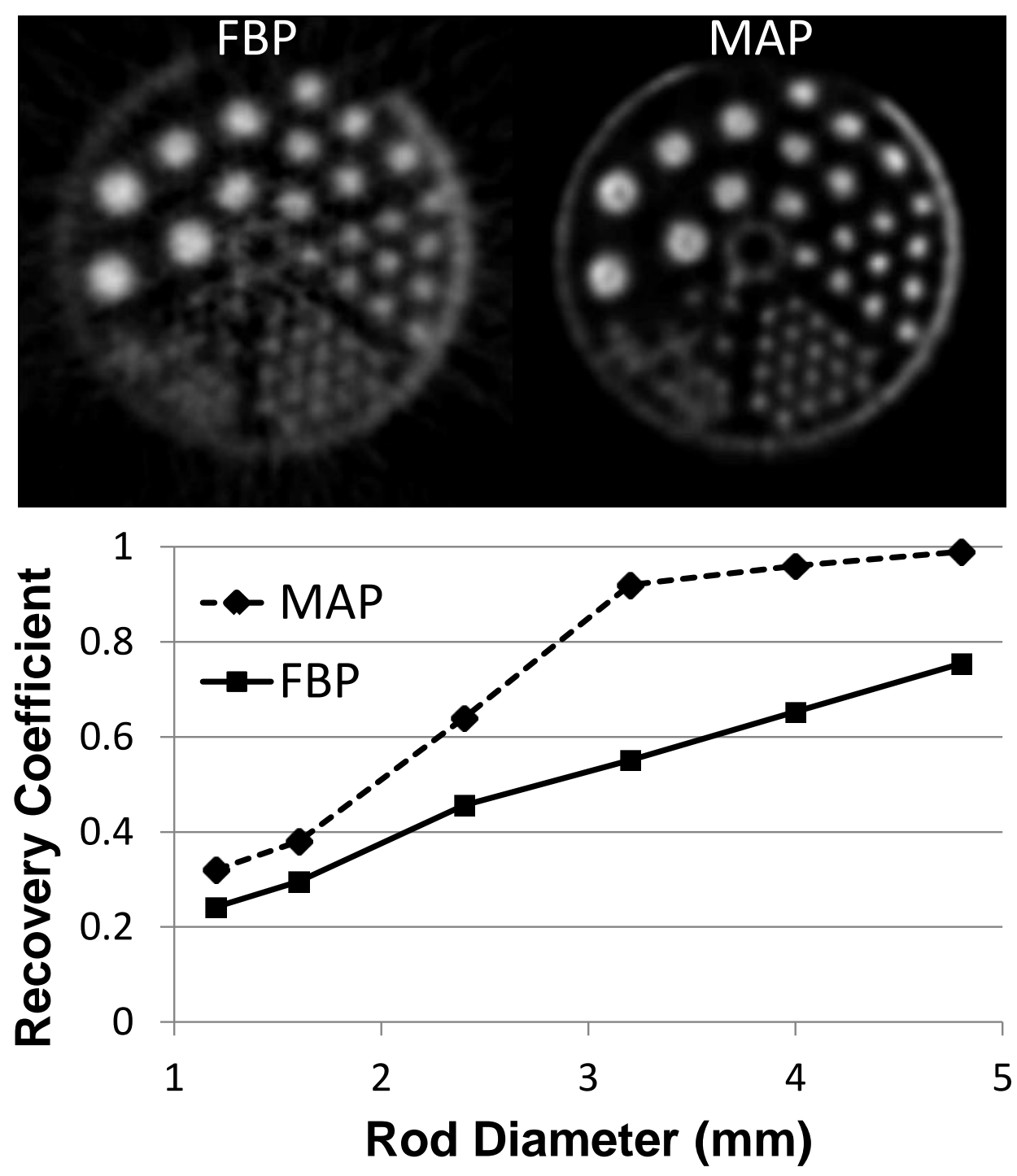

Figure 6-1. Transaxial view of attenuation-corrected phantom images reconstructed with a) FBP and b) MAP and the recovery coefficient curves generated from them $(\mathbf{c})$.

Representative OSEM-MAP and FBP reconstructed PET images of a mouse heart with and without cardiac gating are shown in Figure 6-2. Each image shown is the last frame of the dynamic PET scan. OSEM-MAP reconstructed images (panels (a) and (b)) clearly show a reduced amount of contamination in the LVBP compared to the same data reconstructed with FBP (panels (c) and (d)) regardless of whether cardiac gating was 

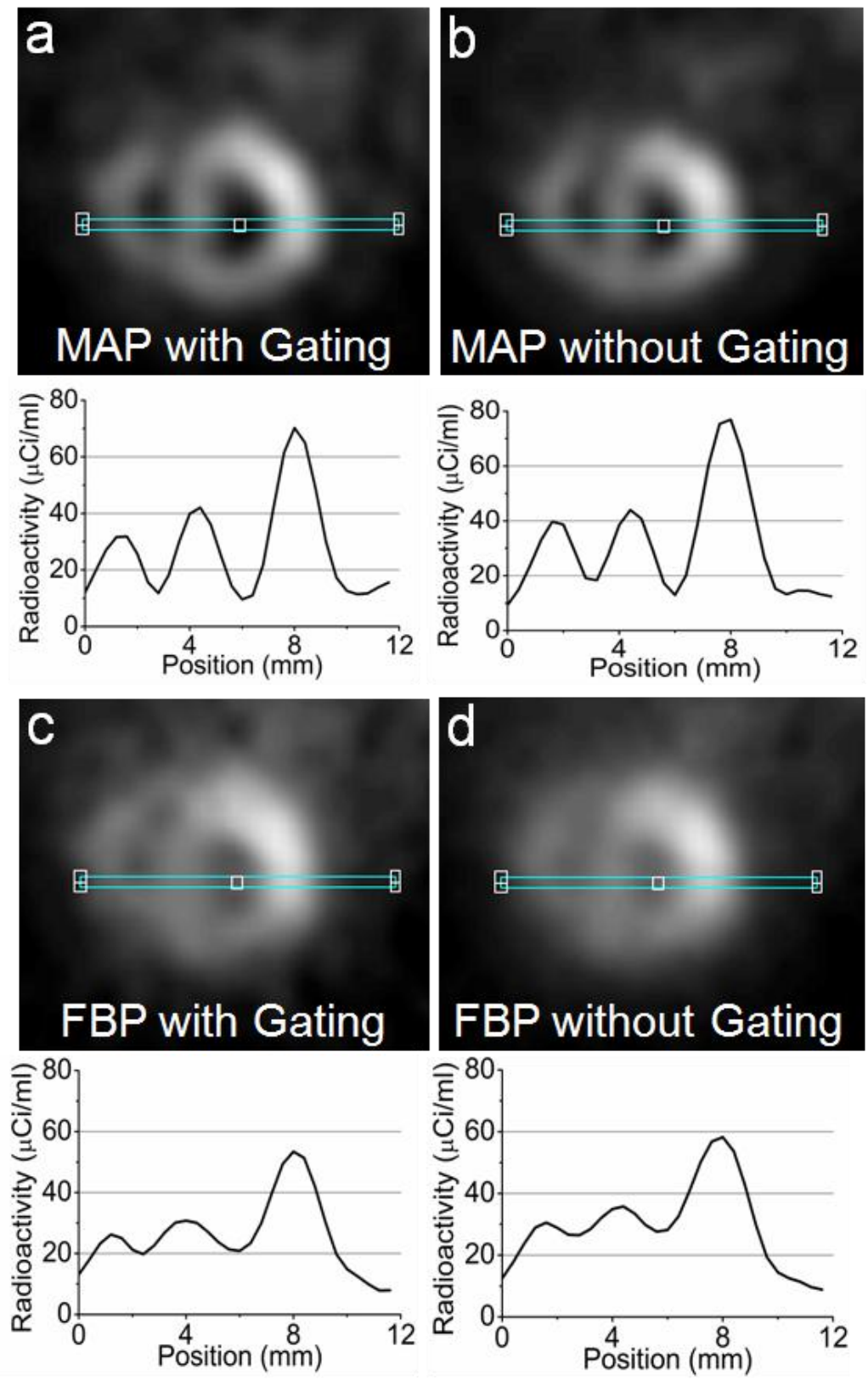

Figure 6-2. Transverse FDG PET images of a mouse heart reconstructed using a OSEM-MAP with cardiac gating, b OSEM-MAP without cardiac gating, $\mathbf{c}$ FBP with cardiac gating, and $\mathbf{d}$ FBP without gating. Common line profiles through the LVBP are shown below each image. Only cardiac-gated, OSEM-MAP reconstructions produced images that allowed accurate measurement of blood radioactivity concentration in the LVBP. 
employed. Qualitatively, line profiles through the heart show a higher myocardium-toLVBP contrast for OSEM-MAP reconstructed images compared to FBP.

LVBP radioactivity concentrations are dependent on both image reconstruction algorithm and whether cardiac gating is employed. Cardiac-gated images show an improved contrast between the blood pool and the myocardium compared to non-gated images. Images reconstructed by FBP appear to be noisier than the corresponding OSEM-MAP reconstructed images despite the fact that both images have the same resolution. Only cardiac-gated OSEM-MAP reconstructed images allowed accurate measurement of LVBP radioactivity concentrations.

Figure 6-3 shows IDIFs derived from the PET images shown in panels (a), (c), and (d) of the previous figure. The IDIF measured from the image reconstructed with FBP (black curve) shows significant spillover contamination compared to the IDIF derived from the OSEM-MAP reconstructed image (gray curve) using common LVBP ROIs. The IDIF derived from the non-gated FBP reconstruction shows the greatest amount of spillover (dotted black line). The peaks of the three curves are similar in magnitude due to the application of the $\mathrm{RC}$ and a lack of spillover contamination in the LVBP at early time points. The square and circle symbols, which represent the radioactivity concentrations of sampled blood taken at 43 and 56 min respectively, are only in close agreement with activity concentrations of the IDIF derived from the OSEMMAP reconstructed, cardiac-gated image.

The Bland-Altman graph shown in Figure 6-4 serves to assess the agreement between PET image-derived and physically sampled blood radioactivity concentrations. Bland-Altman analysis revealed a mean difference of $0.06 \mu \mathrm{Ci} / \mathrm{mL}$, which is 


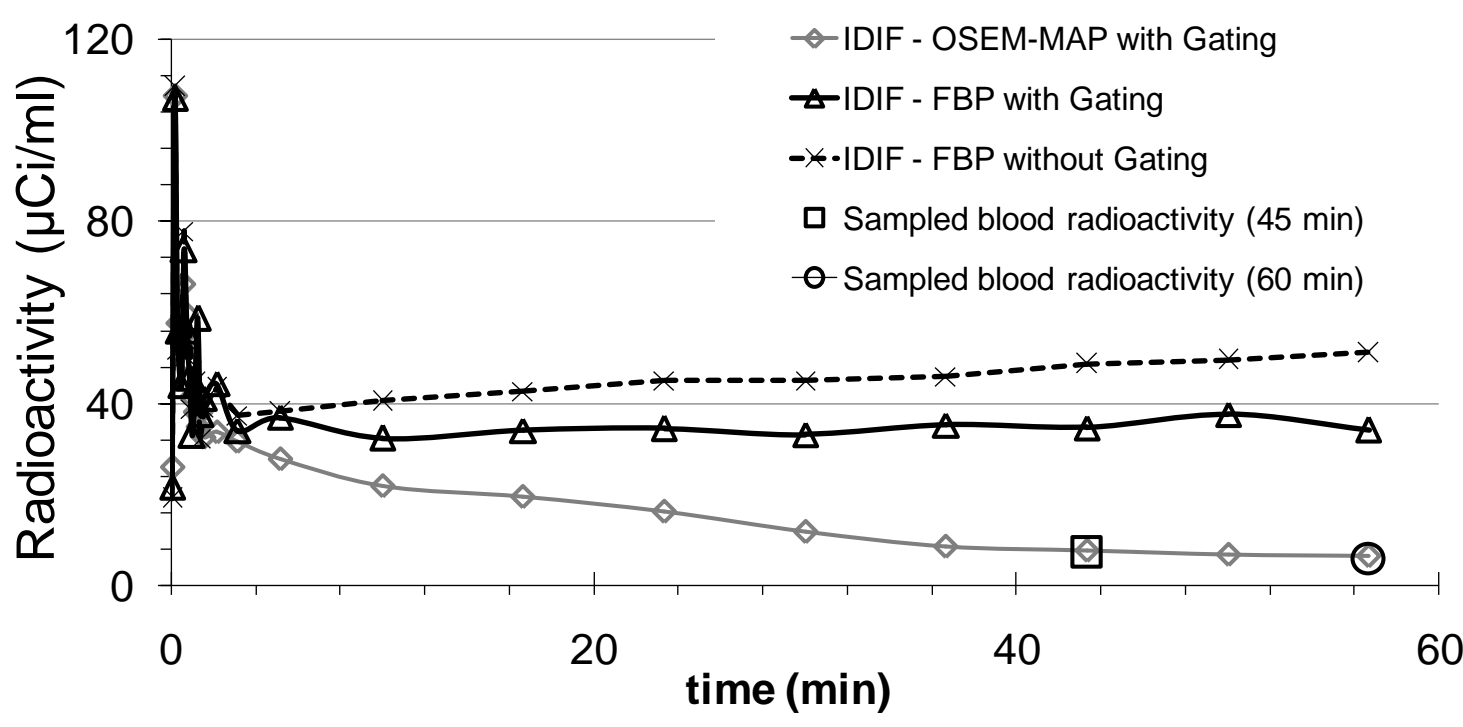

Figure 6-3. Representative IDIFs, derived from a common LVBP ROI drawn on PET images shown in panels (a), (c), and (d) of the previous figure are shown. The square and circular boxes, which represent manually sampled blood activity concentrations taken at 43 and 56 minutes respectively, are only in close agreement with activity concentrations of the IDIF derived from the OSEM-MAP reconstructed, cardiac-gated image (solid gray line). The IDIF derived from the non-gated, FBP reconstructed image (black dotted line) suffered the highest amount of spillover contamination.

approximately $1 \%$ of the average of all measured values, and a precision of $0.14 \mu \mathrm{Ci} / \mathrm{mL}$ (standard deviation of differences). None of the data points fell beyond the lower or upper limits of agreement, which were -0.22 and 0.34 , respectively. As determined by the paired $t$-test, image-derived blood radioactivity concentration values were not significantly different from sampled blood values $(P=0.97)$.

As a final step of validation, we compared the mean FDG influx constant $\left(\mathrm{K}_{\mathrm{i}}\right)$ in the myocardium computed from an IDIF to a published value of $\mathrm{K}_{\mathrm{i}}$ in the same tissue computed using an input function constructed from arterial blood samples. The mean $\mathrm{K}_{\mathrm{i}}$ for myocardial tissue measured here was $0.11 \pm 0.03$ which is in close agreement with the published value of $0.14 \pm 0.06$ [29]. Using the Student's $t$-test, we determined the difference between $\mathrm{K}_{\mathrm{i}}$ values to be non-significant $(P=0.19)$. 


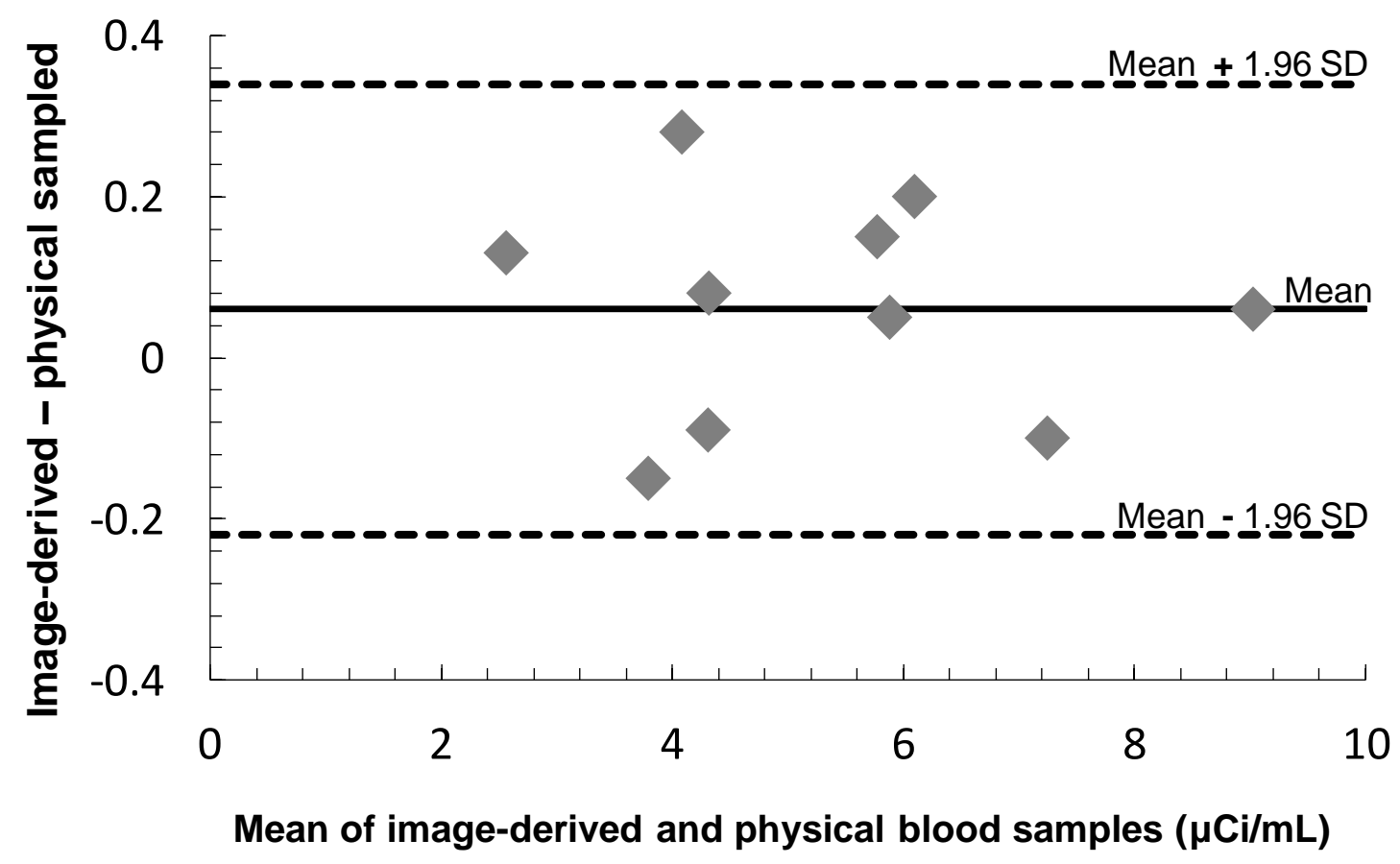

Figure 6-4. Bland-Altman plot showing the agreement between image-derived (corrected for attenuation and PV effects) and physically sampled blood radioactivity concentrations in five mice. The mean difference was $-0.06 \mu \mathrm{Ci} / \mathrm{mL}$ (solid line) and the lower and upper limits of agreement (dashed lines) were -0.22 and 0.34 , respectively.

\section{Conclusion}

Employing cardiac gating and a high-resolution iterative reconstruction algorithm (OSEM-MAP) improves PET image quality, reduces PV effects, and minimizes spillover contamination from the myocardium to the LVBP compared to non-gated, FBP reconstructed images. We validated the ability to accurately measure blood pool radioactivity concentrations from the left ventricle in a normal mouse using imagederived techniques. Bland-Altman analysis revealed a negligible systematic bias between image-derived and physically sampled blood radioactivity concentrations for five mice studied. Furthermore, we showed that the FDG influx constant in the myocardium computed using three-compartment modeling based on an IDIF was not statistically different from reported values obtained with a physically sampled input function. This 
imaging method may be useful for surveillance of neutrophil activation in the context of inflammatory lung diseases and its noninvasive nature makes it more accessible for use in the preclinical setting. 


\section{APPENDIX B: Mechanism of Lung Tumor Induction by Urethane}

Murine lung tumor models induced by injections of constituents of tobacco smoke have proven to be useful because of their reproducibility, histological similarity with human lung adenocarcinoma (AC), and realistic tumor progression. Urethane has been extensively used to model multistage human lung carcinogenesis in A/J and FVB mice $[74,110,111]$. In the $\mathrm{A} / \mathrm{J}$ urethane model, lung tumors proceed through hyperplastic and adenoma stages, ultimately developing into $\mathrm{AC}$ with similar molecular and histologic features seen in human AC, especially those found in never-smokers [112]. Also, as commonly seen with human AC, these lung tumors arise from alveolar type II epithelial cells [112]. An attractive benefit of the A/J and FVB mouse-urethane models is that lung tumor induction occurs in about half the time of the cigarette smoking model [113]. Furthermore, through depletion studies, macrophages have been shown to promote lung tumorigenesis, influence mean tumor diameter, and contribute to the local expansion of tumor-associated blood vessels in the FVB-urethane model [15].

Previously published work has shown that lung carcinogenesis in the urethane (ethyl carbamate, EC) model is mediated by itsbioconversion into vinyl carbamate (VC) by the cytochrome P450 enzyme CYP2E1 $[114,115]$. VC is susceptible to chemical oxidation leading to the formation of $\mathrm{VC}$ epoxide, a strong mutagenic and carcinogenic species capable of modifying DNA and producing mutations [116]. An alternative enzymatic pathway exists in which EC is broken down into ammonia, carbon dioxide, and ethanol by the enzyme carboxylesterase. Carboxylesterase competes with CYP2E1 in the bioconversion of EC to either its potentially toxic form or its harmless metabolites. The relative rates of activity and concentrations of carboxylesterase and CYP2E1 in the 
lung tissue are most likely the major determinants of the host's susceptibility to urethaneinduced tumorigenesis.

Inbred strains of mice exhibit differing susceptibilities to formation of lung tumors induced by carcinogens including EC. Susceptible mouse strains such as A/J and FVB have elevated levels of CYP2E1 in their lungs compared to C57BL/6 mice and have been shown to develop significanly more lung tumors as a result of EC treatment. A recent study showed a significant reduction in urethane-induced lung tumor formation in CYP2E1-knockout mice compared to wildtype controls [117]. Other work demonstrated that a chemical inhibitor of the CYP2E1 enzyme resulted in fewer EC-induced mutations and lung tumors [115]. In addition to lungs, other tissues such as skin and lymphoid tissue develop tumors after EC treatment. However, a latent period of about 1 year is required for tumor development, presumably due to lower levels of CYP2E1 in these tissues. In our studies, we found that $100 \%$ of mice treated with EC developed lung tumors by 20 weeks. Upon necrosy, tumors were not found in any other organ. In contrast, no lung tumors were detected out to 24 weeks in saline-injected mice. 


\section{APPENDIX C: Liposome Titration}

Prior to examining liposome uptake by BM-derived MØ monolayers, a titration study was first performed to determine the optimal liposome concentration that approached the saturation level, but was slightly below it. As fully described in 3.2.4, BM-derived macrophages were seeded into $60 \mathrm{~mm}$ Petri dishes at a density of $1 \times 10^{6}$ cells per plate and stimulated with IL-4 and IL-13 to generate an M2-polarized phenotype. Cells stimulated with these cytokines were anticipated to exhibit the highest uptake of $\mathrm{Man}_{3}$-liposomes due to their over-expression of mannose receptor, which was desired for this titration study. Following the cytokine stimulation period, the cells were washed and incubated with $0,2.4,24,48,240,480$, or 960 nmoles of lipid per $\mathrm{mL}$ of either $\mathrm{Man}_{3}$ or plain liposomes for $90 \mathrm{~min}$ at $37^{\circ} \mathrm{C}$. The range of liposome concentrations and the incubation time was chosen was based on a similar study that measured mannosylated liposome uptake by mouser peritoneal macrophages [77]. After the incubation time, the medium was pipetted-off and the cells were washed three times with cold PBS. The adherent cells were dislodged with trypsin and washed an additional three times with MACS buffer before fixation by Cytofix (BD Biosciences). Cellassociated $\mathrm{DiO}$ was measured by flow cytometry. As shown in Figure 8-1, the mean fluorescence intensity of cell-associated $\mathrm{DiO}$ quickly reached a plateau for plain liposomes. In contrast, $\mathrm{Man}_{3}$-liposomes did not exhibit this plateau behavior and were found to be associated with the cells in about 2 to 4 -fold greater amounts compared to plain liposomes. The liposome concentration that yielded the greatest difference in uptake between $\mathrm{Man}_{3}$ and plain liposomes was of 960 nmoles of lipid per mL: the highest 
concentration tested. Because it resulted in the greatest signal separation, this liposome concentration was used for all future in vitro uptake studies.

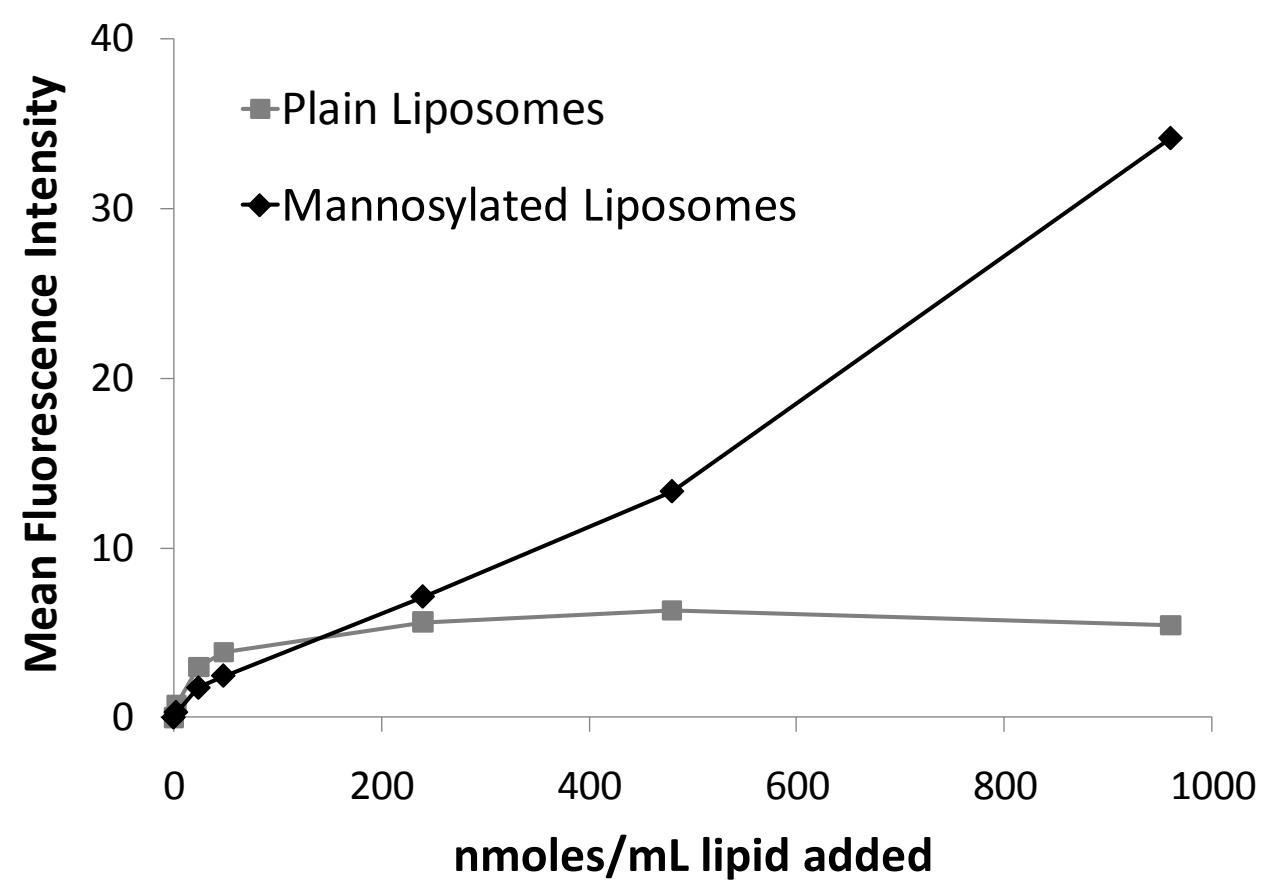

Figure 8-1. Dose dependent increases in M2 macrophage uptake of DiO-labeled plain ( $\mathbf{})$ and mannosylated ( $\bullet$ ) liposomes. The uptake of DiO-labeled liposomes was calculated as the geometric mean of fluorescence in the green channel after the background fluorescence was subtracted. 


\section{REFERENCES}

1. Jones, H.A., et al., In vivo assessment of lung inflammatory cell activity in patients with COPD and asthma. Eur Respir J, 2003. 21(4): p. 567-73.

2. Beeh, K.M., et al., Neutrophil chemotactic activity of sputum from patients with COPD: role of interleukin 8 and leukotriene B4. Chest, 2003. 123(4): p. 1240-7.

3. Gane, J. and R. Stockley, Mechanisms of neutrophil transmigration across the vascular endothelium in COPD. Thorax, 2011.

4. Hayes, E., et al., The cystic fibrosis neutrophil: a specialized yet potentially defective cell. Arch Immunol Ther Exp (Warsz), 2011. 59(2): p. 97-112.

5. Ruparelia, P., et al., 99mTechnetium-labelled neutrophil scanning in pneumonia. Thorax, 2009. 64(1): p. 92.

6. Ruparelia, P., et al., Quantification of neutrophil migration into the lungs of patients with chronic obstructive pulmonary disease. Eur J Nucl Med Mol Imaging, 2011. 38(5): p. 911-9.

7. Dumarey, N., et al., Imaging infection with 18F-FDG-labeled leukocyte PET/CT: initial experience in 21 patients. J Nucl Med, 2006. 47(4): p. 625-32.

8. Marin, L., et al., Chronic obstructive pulmonary disease: patho-physiology, current methods of treatment and the potential for simvastatin in disease management. Expert Opin Drug Deliv, 2011. 8(9): p. 1205-20.

9. Burgel, P.R., et al., [Structural abnormalities and inflammation in COPD: a focus on small airways]. Rev Mal Respir, 2011. 28(6): p. 749-60.

10. Murphy, B.S., et al., Characterization of macrophage activation states in patients with cystic fibrosis. J Cyst Fibros, 2010. 9(5): p. 314-22. 
11. Conese, M., Cystic fibrosis and the innate immune system: therapeutic implications. Endocr Metab Immune Disord Drug Targets, 2011. 11(1): p. 8-22.

12. Sica, A., Role of tumour-associated macrophages in cancer-related inflammation. Exp Oncol, 2010. 32(3): p. 153-8.

13. Mantovani, A. and A. Sica, Macrophages, innate immunity and cancer: balance, tolerance, and diversity. Curr Opin Immunol, 2010. 22(2): p. 231-7.

14. Zhang, B., Yao, G., Zhang, Y., Gao, J., Yang, B., Rao, Z., Gao, J., M2-Polarized tumor-associated macrophages are associated with poor prognoses resulting from accelerated lymphangiogenesis in lung adenocarcinoma. Clinics, 2011. 66(11): p. 1878-1886.

15. Zaynagetdinov, R., et al., A critical role for macrophages in promotion of urethaneinduced lung carcinogenesis. J Immunol, 2011. 187(11): p. 5703-11.

16. Haslett, C., et al., 111Indium-labeled neutrophil migration into the lungs of bleomycin-treated rabbits assessed noninvasively by external scintigraphy. Am Rev Respir Dis, 1989. 140(3): p. 756-63.

17. Lichter, J.P., et al., The in vivo behavior of granulocytes labeled with indium-111 in a canine model of pneumococcal pneumonia. Am Rev Respir Dis, 1984. 129(4): p. 602-6.

18. de Prost, N., M.R. Tucci, and M.F. Melo, Assessment of lung inflammation with 18FFDG PET during acute lung injury. AJR Am J Roentgenol, 2010. 195(2): p. 292300.

19. Schroeder, T., et al., Modeling pulmonary kinetics of 2-deoxy-2-[18F]fluoro-Dglucose during acute lung injury. Acad Radiol, 2008. 15(6): p. 763-75. 
20. Zhuang, H.M. and A. Alavi, 18-fluorodeoxyglucose positron emission tomographic imaging in the detection and monitoring of infection and inflammation. Seminars in Nuclear Medicine, 2002. 32(1): p. 47-59.

21. Zhou, Z., et al., Molecular imaging of lung glucose uptake after endotoxin in mice. Am J Physiol Lung Cell Mol Physiol, 2005. 289(5): p. L760-8.

22. Jones, H.A., et al., Dissociation of neutrophil emigration and metabolic activity in lobar pneumonia and bronchiectasis. Eur Respir J, 1997. 10(4): p. 795-803.

23. Hartwig, W., et al., Neutrophil metabolic activity but not neutrophil sequestration reflects the development of pancreatitis-associated lung injury. Crit Care Med, 2002. 30(9): p. 2075-82.

24. Hansson, L., et al., Glucose utilisation in the lungs of septic rats. Eur J Nucl Med, 1999. 26(10): p. 1340-4.

25. Jones, H.A., et al., In vivo measurement of neutrophil activity in experimental lung inflammation. Am J Respir Crit Care Med, 1994. 149(6): p. 1635-9.

26. Schuster, D.P., J. Kozlowski, and L. Hogue, Imaging lung inflammation in a murine model of Pseudomonas infection: a positron emission tomography study. Exp Lung Res, 2003. 29(1): p. 45-57.

27. Chen, D.L., M.A. Mintun, and D.P. Schuster, Comparison of methods to quantitate 18F-FDG uptake with PET during experimental acute lung injury. J Nucl Med, 2004. 45(9): p. 1583-90.

28. Tantawy, M.N. and T.E. Peterson, Simplified [18F]FDG image-derived input function using the left ventricle, liver, and one venous blood sample. Mol Imaging, 2010. 9(2): p. 76-86. 
29. Fang, Y.H. and R.F. Muzic, Jr., Spillover and partial-volume correction for imagederived input functions for small-animal 18F-FDG PET studies. J Nucl Med, 2008. 49(4): p. 606-14.

30. Zheng, X., et al., A hybrid clustering method for ROI delineation in small-animal dynamic PET images: application to the automatic estimation of FDG input functions. IEEE Trans Inf Technol Biomed, 2011. 15(2): p. 195-205.

31. Kim, J., et al., Minimally invasive method of determining blood input function from PET images in rodents. J Nucl Med, 2006. 47(2): p. 330-6.

32. Wu, H.M., et al., In vivo quantitation of glucose metabolism in mice using smallanimal PET and a microfluidic device. J Nucl Med, 2007. 48(5): p. 837-45.

33. Huang, S.C., et al., Investigation of a new input function validation approach for dynamic mouse microPET studies. Mol Imaging Biol, 2004. 6(1): p. 34-46.

34. Ferl, G.Z., et al., Estimation of the 18F-FDG input function in mice by use of dynamic small-animal PET and minimal blood sample data. J Nucl Med, 2007. 48(12): p. 2037-45.

35. Laforest, R., et al., Measurement of input functions in rodents: challenges and solutions. Nucl Med Biol, 2005. 32(7): p. 679-85.

36. Locke, L.W., et al., A Novel Neutrophil-Specific PET Imaging Agent: cFLFLFKPEG-64Cu. J Nucl Med, 2009. 50(5): p. 790-797.

37. Stolin, A., et al. Dual-modality scanner for small animal imaging. in Nuclear Science Symposium Conference Record, 2006. IEEE. 2006.

38. Chow, P.L., F.R. Rannou, and A.F. Chatziioannou, Attenuation correction for small animal PET tomographs. Phys Med Biol, 2005. 50(8): p. 1837-50. 
39. Locke, L.W., S.S. Berr, and B.K. Kundu, Image-Derived Input Function from Cardiac Gated Maximum a Posteriori Reconstructed PET Images in Mice. Mol Imaging Biol, 2011. 13(2): p. 342-7.

40. Strauss, L.G., A. Dimitrakopoulou-Strauss, and U. Haberkorn, Shortened PET data acquisition protocol for the quantification of 18F-FDG kinetics. J Nucl Med, 2003. 44(12): p. 1933-9.

41. Phelps, M.E., et al., Tomographic measurement of local cerebral glucose metabolic rate in humans with (F-18)2-fluoro-2-deoxy-D-glucose: validation of method. Ann Neurol, 1979. 6(5): p. 371-88.

42. Patlak, C.S., R.G. Blasberg, and J.D. Fenstermacher, Graphical evaluation of bloodto-brain transfer constants from multiple-time uptake data. J Cereb Blood Flow Metab, 1983. 3(1): p. 1-7.

43. Babich, J.W., et al., A novel high affinity chemotactic peptide antagonist for infection imaging. J Nucl Med, 1997. 38: p. 268P.

44. Babich, J.W., et al., A novel high affinity peptide antagonist for infection imaging. Journal of Nuclear Medicine, 1997. 38: p. 268P.

45. Zhang, Y., et al., Synthesis of novel neutrophil-specific imaging agents for Positron Emission Tomography (PET) imaging. Bioorg Med Chem Lett, 2007. 17(24): p. 6876-8.

46. Ferrante, A. and Y.H. Thong, Simultaneous preparation of mononuclear and polymorphonuclear leucocytes from horse blood on Ficoll-Hypaque medium. Journal of Immunological Methods, 1980. 34(4): p. 279-85. 
47. Sullivan, G.W., et al., Cyclic AMP-dependent inhibition of human neutrophil oxidative activity by substituted 2-propynylcyclohexyl adenosine A(2A) receptor agonists. British Journal of Pharmacology, 2001. 132(5): p. 1017-1026.

48. Dechatelet, L.R., et al., Mechanism of the Luminol-Dependent Chemi-Luminescence of Human-Neutrophils. Journal of Immunology, 1982. 129(4): p. 1589-1593.

49. Baker, H.C., R.i. Lindsey, and S.H. Weisbroth, The Laboratory Rat. 1980, New York: New York:

Academic Press. 257.

50. Handl, H.L., et al., Hitting multiple targets with multimeric ligands. Expert Opinion on Therapeutic Targets, 2004. 8(6): p. 565-586.

51. Rini, J.N., et al., PET with FDG-labeled leukocytes versus scintigraphy with 111Inoxine-labeled leukocytes for detection of infection. Radiology, 2006. 238(3): p. 97887.

52. Babich, J.W., et al., Localization of radiolabeled chemotactic peptide at focal sites of Escherichia coli infection in rabbits: evidence for a receptor-specific mechanism.[see comment]. Journal of Nuclear Medicine, 1997. 38(8): p. 1316-22.

53. Fischman, A.J., et al., Imaging focal sites of bacterial infection in rats with indium111-labeled chemotactic peptide analogs. J Nucl Med, 1991. 32(3): p. 483-491.

54. Allavena, P., et al., The inflammatory micro-environment in tumor progression: the role of tumor-associated macrophages. Crit Rev Oncol Hematol, 2008. 66(1): p. 1-9.

55. Bingle, L., N.J. Brown, and C.E. Lewis, The role of tumour-associated macrophages in tumour progression: implications for new anticancer therapies. J Pathol, 2002. 196(3): p. 254-65. 
56. Murdoch, C., et al., The role of myeloid cells in the promotion of tumour angiogenesis. Nat Rev Cancer, 2008. 8(8): p. 618-31.

57. Siveen, K.S. and G. Kuttan, Role of macrophages in tumour progression. Immunol Lett, 2009. 123(2): p. 97-102.

58. Robinson, S.C., et al., A chemokine receptor antagonist inhibits experimental breast tumor growth. Cancer Res, 2003. 63(23): p. 8360-5.

59. Zeisberger, S.M., et al., Clodronate-liposome-mediated depletion of tumourassociated macrophages: a new and highly effective antiangiogenic therapy approach. Br J Cancer, 2006. 95(3): p. 272-81.

60. Banciu, M., et al., Antitumor activity of liposomal prednisolone phosphate depends on the presence of functional tumor-associated macrophages in tumor tissue. Neoplasia, 2008. 10(2): p. 108-17.

61. Pollard, J.W., Tumour-educated macrophages promote tumour progression and metastasis. Nat Rev Cancer, 2004. 4(1): p. 71-8.

62. Melancon, M.P., et al., Targeted imaging of tumor-associated M2 macrophages using a macromolecular contrast agent PG-Gd-NIR813. Biomaterials, 2010. 31(25): p. $6567-73$.

63. Lewis, C.E. and J.W. Pollard, Distinct role of macrophages in different tumor microenvironments. Cancer Res, 2006. 66(2): p. 605-12.

64. Weissleder, R., et al., Cell-specific targeting of nanoparticles by multivalent attachment of small molecules. Nat Biotechnol, 2005. 23(11): p. 1418-23. 
65. Leimgruber, A., et al., Behavior of endogenous tumor-associated macrophages assessed in vivo using a functionalized nanoparticle. Neoplasia, 2009. 11(5): p. 45968, 2 p following 468.

66. Solinas, G., et al., Tumor-associated macrophages (TAM) as major players of the cancer-related inflammation. J Leukoc Biol, 2009. 86(5): p. 1065-73.

67. Redente, E.F., et al., Tumor signaling to the bone marrow changes the phenotype of monocytes and pulmonary macrophages during urethane-induced primary lung tumorigenesis in A/J mice. Am J Pathol, 2007. 170(2): p. 693-708.

68. Mantovani, A., et al., Macrophage polarization: tumor-associated macrophages as a paradigm for polarized M2 mononuclear phagocytes. Trends Immunol, 2002. 23(11): p. 549-55.

69. Allavena, P., et al., Engagement of the mannose receptor by tumoral mucins activates an immune suppressive phenotype in human tumor-associated macrophages. Clin Dev Immunol, 2010. 2010: p. 547179.

70. Lee, S.J., et al., Mannose receptor-mediated regulation of serum glycoprotein homeostasis. Science, 2002. 295(5561): p. 1898-901.

71. McGreal, E.P., L. Martinez-Pomares, and S. Gordon, Divergent roles for C-type lectins expressed by cells of the innate immune system. Mol Immunol, 2004. 41(11): p. 1109-21.

72. Matsui, M., et al., Targeted delivery of oligomannose-coated liposome to the omental micrometastasis by peritoneal macrophages from patients with gastric cancer. Cancer Sci, 2010. 101(7): p. 1670-7. 
73. Kojima, N., et al., Oligomannose-coated liposomes as a therapeutic antigen-delivery and an adjuvant vehicle for induction of in vivo tumor immunity. J Control Release, 2008. 129(1): p. 26-32.

74. Stathopoulos, G.T., et al., Epithelial NF-kappaB activation promotes urethaneinduced lung carcinogenesis. Proc Natl Acad Sci U S A, 2007. 104(47): p. 18514-9.

75. Austyn, J.M. and S. Gordon, F4/80, a monoclonal antibody directed specifically against the mouse macrophage. Eur J Immunol, 1981. 11(10): p. 805-15.

76. Ambarus, C.A., et al., Systematic validation of specific phenotypic markers for in vitro polarized human macrophages. J Immunol Methods, 2011.

77. Muller, C.D. and F. Schuber, Neo-mannosylated liposomes: synthesis and interaction with mouse Kupffer cells and resident peritoneal macrophages. Biochim Biophys Acta, 1989. 986(1): p. 97-105.

78. Petersen, A.L., et al., 64Cu loaded liposomes as positron emission tomography imaging agents. Biomaterials, 2011. 32(9): p. 2334-41.

79. Yamazaki, T., K. Nagata, and Y. Kobayashi, Cytokine production by M-CSF-and GM-CSF-induced mouse bone marrow-derived macrophages upon coculturing with late apoptotic cells. Cell Immunol, 2008. 251(2): p. 124-30.

80. Kigerl, K.A., et al., Identification of two distinct macrophage subsets with divergent effects causing either neurotoxicity or regeneration in the injured mouse spinal cord. J Neurosci, 2009. 29(43): p. 13435-44.

81. Sica, A., A. Saccani, and A. Mantovani, Tumor-associated macrophages: a molecular perspective. Int Immunopharmacol, 2002. 2(8): p. 1045-54. 
82. Gocheva, V., et al., IL-4 induces cathepsin protease activity in tumor-associated macrophages to promote cancer growth and invasion. Genes Dev, 2010. 24(3): p. $241-55$.

83. Mosser, D.M. and J.P. Edwards, Exploring the full spectrum of macrophage activation. Nat Rev Immunol, 2008. 8(12): p. 958-69.

84. Mantovani, A., et al., The chemokine system in diverse forms of macrophage activation and polarization. Trends Immunol, 2004. 25(12): p. 677-86.

85. Luo, Y., et al., Targeting tumor-associated macrophages as a novel strategy against breast cancer. J Clin Invest, 2006. 116(8): p. 2132-2141.

86. Chen, P., et al., Tumor-Associated Macrophages Promote Angiogenesis and Melanoma Growth via Adrenomedullin in a Paracrine and Autocrine Manner. Clin Cancer Res, 2011.

87. Sica, A., et al., Macrophage polarization in tumour progression. Semin Cancer Biol, 2008. 18(5): p. 349-55.

88. Litzinger, D.C. and L. Huang, Amphipathic poly(ethylene glycol) 5000-stabilized dioleoylphosphatidylethanolamine liposomes accumulate in spleen. Biochim Biophys Acta, 1992. 1127(3): p. 249-54.

89. Loening, A.M. and S.S. Gambhir, AMIDE: a free software tool for multimodality medical image analysis. Mol Imaging, 2003. 2(3): p. 131-7.

90. Magota, K., et al., Performance characterization of the Inveon preclinical smallanimal PET/SPECT/CT system for multimodality imaging. Eur J Nucl Med Mol Imaging, 2011. 38(4): p. 742-52. 
91. Wijagkanalan, W., et al., Efficient targeting to alveolar macrophages by intratracheal administration of mannosylated liposomes in rats. J Control Release, 2008. 125(2): p. 121-30.

92. Wijagkanalan, W., et al., Enhanced anti-inflammation of inhaled dexamethasone palmitate using mannosylated liposomes in an endotoxin-induced lung inflammation model. Mol Pharmacol, 2008. 74(5): p. 1183-92.

93. Bauer, A.K., et al., Targeted deletion of nrf2 reduces urethane-induced lung tumor development in mice. PLoS One, 2011. 6(10): p. e26590.

94. Redente, E.F., et al., Tumor Progression Stage and Anatomical Site Regulate TumorAssociated Macrophage and Bone Marrow-Derived Monocyte Polarization. Am J Pathol, 2010.

95. Raveh, D., et al., Th1 and Th2 cytokines cooperate to stimulate mannose-receptormediated phagocytosis. J Leukoc Biol, 1998. 64(1): p. 108-13.

96. Duluc, D., et al., Interferon-gamma reverses the immunosuppressive and protumoral properties and prevents the generation of human tumor-associated macrophages. Int J Cancer, 2009. 125(2): p. 367-73.

97. Srinivas, S.M., et al., A recovery coefficient method for partial volume correction of PET images. Ann Nucl Med, 2009. 23(4): p. 341-8.

98. Branco, S., S. Jan, and P. Almeida. Respiratory motion modeling in small animal PET using GATE. in Nuclear Science Symposium Conference Record, 2008. NSS '08. IEEE. 2008.

99. Singodia, D., et al., Investigations into an alternate approach to target mannose receptors on macrophages using 4-sulfated $N$-acetyl galactosamine more efficiently 
in comparison with mannose-decorated liposomes: An application in drug delivery. Nanomedicine, 2011.

100. Ishida, T., H. Harashima, and H. Kiwada, Liposome clearance. Biosci Rep, 2002. 22(2): p. 197-224.

101. Xiao, L., et al., Synthesis of the Cyanine 7 labeled neutrophil-specific agents for noninvasive near infrared fluorescence imaging. Bioorg Med Chem Lett, 2010. 20(12): p. 3515-7.

102. Schroit, A.J. and I.J. Fidler, Effects of liposome structure and lipid composition on the activation of the tumoricidal properties of macrophages by liposomes containing muramyl dipeptide. Cancer Res, 1982. 42(1): p. 161-7.

103. Fidler, I.J., et al., Pulmonary localization of intravenously injected liposomes. Recent Results Cancer Res, 1980. 75: p. 246-51.

104. Immordino, M.L., F. Dosio, and L. Cattel, Stealth liposomes: review of the basic science, rationale, and clinical applications, existing and potential. Int $\mathrm{J}$ Nanomedicine, 2006. 1(3): p. 297-315.

105. Poiani, G.J., et al., Liposome encapsulation improves the effect of antifibrotic agent in rat lung fibrosis. Am J Respir Crit Care Med, 1994. 150(6 Pt 1): p. 1623-7.

106. Bland, J.M. and D.G. Altman, Measuring agreement in method comparison studies. Stat Methods Med Res, 1999. 8(2): p. 135-60.

107. Chin, B.B., et al., Left ventricular functional assessment in mice: feasibility of high spatial and temporal resolution ECG-gated blood pool SPECT. Radiology, 2007. 245(2): p. 440-8. 
108. Phelps, M.E., PET: Molecular Imaging and Its Biological Applications. 1st ed. 2004: Springer. 624.

109. Hoff, J., Kinetic Modelling

Small Animal Imaging, F. Kiessling and B.J. Pichler, Editors. 2011, Springer Berlin Heidelberg. p. 387-403.

110. Shimkin, M.B. and G.D. Stoner, Lung tumors in mice: application to carcinogenesis bioassay. Adv Cancer Res, 1975. 21: p. 1-58.

111. Meuwissen, R. and A. Berns, Mouse models for human lung cancer. Genes Dev, 2005. 19(6): p. 643-64.

112. Stearman, R.S., et al., Analysis of orthologous gene expression between human pulmonary adenocarcinoma and a carcinogen-induced murine model. Am J Pathol, 2005. 167(6): p. 1763-75.

113. Witschi, H., Tobacco smoke-induced lung cancer in animals--a challenge to toxicology (?). Int J Toxicol, 2007. 26(4): p. 339-44.

114. Hernandez, L.G. and P.G. Forkert, In vivo mutagenicity of vinyl carbamate and ethyl carbamate in lung and small intestine of F1 (Big Blue $x A / J)$ transgenic mice. Int J Cancer, 2007. 120(7): p. 1426-33.

115. Forkert, P.G., Mechanisms of lung tumorigenesis by ethyl carbamate and vinyl carbamate. Drug Metab Rev, 2010. 42(2): p. 355-78.

116. Hernandez, L.G. and P.G. Forkert, Inhibition of vinyl carbamate-induced lung tumors and Kras 2 mutations by the garlic derivative diallyl sulfone. Mutat Res, 2009. 662(1-2): p. 16-21. 
117. Ghanayem, B.I., Inhibition of urethane-induced carcinogenicity in cyp2e1-/- in comparison to cyp2e1+/+ mice. Toxicol Sci, 2007. 95(2): p. 331-9. 